\title{
SECOND-ORDER STRONGLY ELLIPTIC OPERATORS ON LIE GROUPS WITH HÖLDER CONTINUOUS COEFFICIENTS
}

\author{
A. F. M. TER ELST and DEREK W. ROBINSON
}

(Received 15 August 1996)

Communicated by A. H. Dooley

\begin{abstract}
Let $G$ be a connected Lie group with Lie algebra $\mathfrak{g}$ and $a_{1}, \ldots, a_{d^{\prime}}$ an algebraic basis of $\mathfrak{g}$. Further let $A_{i}$ denote the generators of left translations, acting on the $L_{p}$-spaces $L_{p}(G ; d g)$ formed with left Haar measure $d g$, in the directions $a_{i}$. We consider second-order operators

$$
-\sum_{i, j=1}^{d^{\prime}} A_{i} c_{i j} A_{j}+\sum_{i=1}^{d^{\prime}}\left(c_{i} A_{i}+A_{i} c_{i}^{\prime}\right)+c_{0} I
$$

in divergence form corresponding to a quadratic form with complex coefficients, bounded Hölder continuous principal coefficients $c_{i j}$ and lower order coefficients $c_{i}, c_{i}^{\prime}, c_{0} \in L_{\infty}$ such that the matrix $C=\left(c_{i j}\right)$ of principal coefficients satisfies the subellipticity condition

$$
\Re C=2^{-1}\left(C+C^{*}\right) \geq \mu I>0
$$
\end{abstract}

uniformly over $G$.

We discuss the hierarchy relating smoothness properties of the coefficients of $H$ with smoothness of the kernel and smoothness of the domain of powers of $H$ on the $L_{p}$-spaces. Moreover, we present Gaussian type bounds for the kernel and its derivatives.

Similar theorems are proved for strongly elliptic operators

$$
-\sum_{i, j=1}^{d} c_{i j} A_{i} A_{j}+\sum_{i=1}^{d} c_{i} A_{i}+c_{0} I
$$

in non-divergence form for which the principal coefficients are at least once differentiable.

1991 Mathematics subject classification (Amer. Math. Soc.): primary 35J15; secondary 35K05, 22E30.

\section{Introduction}

Our purpose is to derive regularity properties of second-order operators with complexvalued variable coefficients acting on the $L_{p}$-spaces over a $d$-dimensional connected

(C) 1997 Australian Mathematical Society 0263-6115/97 \$A2.00+0.00 
Lie group $G$. In an earlier paper [ElR6] we examined subelliptic operators and established that uniform continuity of the principal coefficients ensured that the corresponding semigroup kernel satisfied Gaussian bounds and a Hölder continuity property. In the current work we analyze the nexus between smoothness of the coefficients and the kernel.

We principally consider operators in divergence form,

$$
H=-\sum_{i, j=1}^{d^{\prime}} A_{i} c_{i j} A_{j}+\sum_{i=1}^{d}\left(c_{i} A_{i}+A_{i} c_{i}^{\prime}\right)+c_{0} I,
$$

with complex coefficients $c_{i j}, c_{i}, c_{i}^{\prime}, c_{0} \in L_{\infty}$. The $A_{i}$ denote the generators, $A_{i}=$ $d L\left(a_{i}\right)$, of left translations $L$ on the $L_{p}$-spaces in the directions $a_{i}$ of the Lie algebra $\mathfrak{g}$ of $G$ where $a_{1}, \ldots, a_{d^{\prime}}$ is an algebraic basis of $\mathfrak{g}$. We assume the real part of the matrix $C=\left(c_{i j}\right)$ of principal coefficients is strictly positive-definite, that is,

$$
\Re C=2^{-1}\left(C+C^{*}\right) \geq \mu I>0,
$$

in the sense of $d^{\prime} \times d^{\prime}$-matrices, uniformly over $G$. The least upper bound, $\mu_{C}$, of the lower bound $\mu$ is called the ellipticity constant and we set $\|C\|_{\infty}=\sup _{g \in G}\|C(g)\|$ with $\|C(g)\|$ the $l_{2}$-norm of the matrix $C(g)=\left(c_{i j}(g)\right)$. (Here and in the sequel we use the notation of [Rob], [ElR2] and [ElR6].) Most of our results are restricted to strongly elliptic operators, that is, operators for which $a_{1}, \ldots, a_{d^{\prime}}$ is a vector space basis of $\mathfrak{g}$, or to subelliptic operators on stratified groups with $a_{1}, \ldots, a_{d^{\prime}}$ a basis of the generating subspace of the stratification of $\mathfrak{g}$.

The operator $H$, formally given by (1), is first defined on $L_{2}=L_{2}(G ; d g)$, where $d g$ denotes left invariant Haar measure $d g$, as the sectorial operator associated with the form

$$
\varphi \mapsto h(\varphi)=\sum_{i, j=1}^{d^{\prime}}\left(A_{i} \varphi, c_{i j} A_{j} \varphi\right)+\sum_{i=1}^{d^{\prime}}\left(\left(\overline{c_{i}} \varphi, A_{i} \varphi\right)-\left(A_{i} \varphi, c_{i}^{\prime} \varphi\right)\right)+\left(\varphi, c_{0} \varphi\right)
$$

with domain $D(h)=L_{2: 1}^{\prime}=\bigcap_{i=1}^{d^{\prime}} D\left(A_{i}\right)$. Then $h$ is closed and $H$ is a maximal accretive operator which generates a strongly continuous, holomorphic, semigroup $S$ on $L_{2}$ (see, for example, [Kat2, Chapter VI]). Although the semigroup $S$ extends to the $L_{p}$-spaces with $p$ close to 2 by perturbation theory (see [AMT, Section 3.1], [EIR7]) it does not necessarily extend to the spaces with $p$ close to 1 or $p$ very large (see [ACT]). There is no problem if the principal coefficients are real-valued, see [EIR8] but difficulties occur for complex operators. If, however, the principal coefficients $c_{i j}$ are right uniformly continuous then $S$ extends to a holomorphic semigroup on all the spaces $L_{p}(G ; d g), p \in[1, \infty]$. The extension is strongly continuous if $p \in[1, \infty)$ and weakly* continuous if $p=\infty$ (see [AMT], [Aus] and [ElR6]). Moreover, $H$ and $S$ act on the spaces $L_{\hat{p}}=L_{p}(G ; d \hat{g})$ formed with respect to right Haar measure $d \hat{g}$. 
It follows from the results of [ElR6] that if $\operatorname{Re} c_{0}$ is sufficiently large then the semigroup $S$ is uniformly bounded on the $L_{p^{-}}$, or $L_{\hat{p}^{-}}$, spaces with the bound uniform in $p$. Then the fractional powers of $H$ are defined and one of our aims is to identify conditions on the coefficients of $H$ which ensure that the domain of $(I+H)^{n / 2}$ on $L_{p}$, or $L_{\hat{p}}, 1<p<\infty$, coincides with $L_{p ; n}$, or $L_{\hat{p} ; n}$, the subspace of $C^{n}$-elements with respect to left translations. As $S$ is holomorphic this would automatically imply that it maps into the $C^{n}$-subspaces and this in turn would almost imply differentiability of the semigroup kernel $K$ associated with $S$. Conversely, differentiability and smoothness properties of the kernel can be exploited to obtain information about the domain of the generator $H$ and its fractional powers.

The kernel is initially defined as a distribution $K_{t}$ such that

$$
\left(\psi, S_{t} \varphi\right)=\int_{G} d g \overline{\psi(g)} \int_{G} d \hat{h} K_{t}(g ; h) \varphi(h)
$$

for all $\varphi, \psi \in C_{c}^{\infty}(G)$ and $t>0$. But right uniform continuity of the principal coefficients $c_{i j}$ is sufficient to guarantee that $K_{t}$ is Hölder continuous and satisfies Gaussian bounds ([Aus], [ElR6]). Additional smoothness of the coefficients will be shown to imply further smoothness of the kernel.

Before we state the main theorems we introduce a multi-index notation and some spaces of more or less smooth functions. If $n \in \mathbb{N}_{0}$ we set

$$
J_{n}\left(d^{\prime}\right)=\bigoplus_{k=0}^{n}\left\{1, \ldots, d^{\prime}\right\}^{k} \quad \text { and } \quad J\left(d^{\prime}\right)=\bigcup_{n=0}^{\infty} J_{n}\left(d^{\prime}\right)
$$

Then $A^{\alpha}=A_{i_{1}} \cdots A_{i_{n}}$ for $\alpha=\left(i_{1}, \ldots, i_{n}\right)$ and $n=|\alpha|$. It will be clear from the context on which space the $A^{\alpha}$ act. If $|\alpha|=0$ then we set $A^{\alpha}=I$. Furthermore we set $L_{p: n}^{\prime}=\bigcap_{\alpha \in J_{n}\left(d^{\prime}\right)} D\left(A^{\alpha}\right)$ in $L_{p}$ with norm $\|\varphi\|_{p ; n}^{\prime}=\max _{\alpha \in J_{n}\left(d^{\prime}\right)}\left\|A^{\alpha}\right\|_{p}$. For the $L_{\hat{p}}$-spaces we use the notation $L_{\hat{p} ; n}^{\prime}$, etcetera.

Next let $d^{\prime}(\cdot ; \cdot)$ be the right invariant distance canonically associated with the algebraic basis $a_{1}, \ldots, a_{d^{\prime}}$ (see, for example, [Rob, Sections IV.2 and IV.4c]). This distance is characterized by

$$
d^{\prime}(g ; h)=\sup \left\{|\psi(g)-\psi(h)|: \psi \in C_{c}^{\infty}(G), \sum_{i=1}^{d^{\prime}}\left|\left(A_{i} \psi\right)\right|^{2} \leq 1\right\}
$$

where the $\psi$ are real-valued ([Rob, Lemma IV.2.3], or [EIR4, Lemma 4.2]). Other parameters which enter the estimates are the subelliptic modulus $g \mapsto|g|^{\prime}=d^{\prime}(g ; e)$ where $e$ denotes the identity of $G$ and the local dimension $D^{\prime}$, that is, the integer for which the left Haar measure $\left|B^{\prime}(g ; r)\right|$ of the ball $B^{\prime}(g ; r)=\left\{h \in G: d^{\prime}(g ; h)<r\right\}$ satisfies bounds $c^{-1} r^{D^{\prime}} \leq\left|B^{\prime}(e ; r)\right| \leq c r^{D^{\prime}}$ for some $c>0$ and all small $r$. If the algebraic basis $a_{1}, \ldots, a_{d^{\prime}}$ is completed to a vector space basis $a_{1}, \ldots, a_{d}$ of $g$ then 
the associated distance and modulus are denoted by $d(\cdot ; \cdot)$ and $|\cdot|$, respectively, and the corresponding balls by $B(g ; r)$. Note that $D^{\prime}=d$ if $a_{1}, \ldots, a_{d}$ is a vector space basis. In general we omit the prime in the notation for quantities with respect to a vector space basis $a_{1}, \ldots, a_{d}$.

For $v \in\langle 0,1\rangle$ define the (subelliptic) Hölder space $C^{\nu '}(G)$ of continuous functions over $G$ for which

$$
\|\| \varphi\left\|_{C^{\prime \prime}}=\sup _{0<|g|^{\prime} \leq 1}\left(|g|^{\prime}\right)^{-v}\right\|(I-L(g)) \varphi \|_{\infty}
$$

is finite. If $n \in \mathbb{N}$ and $v \in\langle 0,1\rangle$ then we define

$$
C^{n+\nu}{ }^{\prime}(G)=\left\{\varphi \in L_{\infty ; n}^{\prime}: A^{\alpha} \varphi \in C^{\nu '}(G) \text { for all } \alpha \in J_{n}\left(d^{\prime}\right)\right\} .
$$

Note that $L_{\infty ; n+1}^{\prime}(G) \subset C^{n+v}{ }^{\prime}(G)$ for all $n \in \mathbb{N}_{0}$ and $v \in\langle 0,1\rangle$.

The differentiability properties of the kernel, $(g, h) \mapsto K_{t}(g ; h)$, involve derivatives with respect to both variables. Left derivatives with respect to the first variable will be denoted by $A_{i} K_{t}$ and left derivatives with respect to the second by $B_{i} K_{t}$. Multiple derivatives $A^{\alpha} K_{t}, B^{\beta} K_{t}$ etcetera are expressed with the aid of multi-indices.

Our first main result establishes smoothness of the kernel as a consequence of smoothness of the coefficients.

THEOREM 1.1. Let $H$ be a subelliptic second-order operator in divergence form (1). Suppose either

(a) $H$ is strongly elliptic, or

(b) $G$ is stratified and $a_{1}, \ldots, a_{d^{\prime}}$ is a basis for $\mathfrak{g}_{1}$ in the stratification $\left(\mathfrak{g}_{m}\right)_{m \in\{1, \ldots, r\}}$ of $\mathfrak{g}$.

If $c_{i j}, c_{i}, c_{i}^{\prime} \in C^{\nu \prime}(G)$ for some $v \in\langle 0,1\rangle$ and $c_{0} \in L_{\infty}$ then $K_{t}$ is once left differentiable in the first variable and the derivatives are once left differentiable in the second variable. Moreover, for all $\alpha, \beta \in J_{1}\left(d^{\prime}\right)$ and $\kappa>0$ there exist $a, b>0$ and $\omega \geq 0$ such that

$$
\left|\left(A^{\alpha} B^{\beta} K_{t}\right)(g ; h)\right| \leq a t^{-\left(D^{\prime}+|\alpha|+|\beta|\right) / 2} e^{\omega t} e^{-b\left(\left|g h^{-1}\right|^{\prime}\right)^{2} t^{-1}}
$$

for $g, h \in G$ and $t>0$ and

$$
\begin{aligned}
& \left|\left(A^{\alpha} B^{\beta} K_{t}\right)\left(k^{-1} g ; l^{-1} h\right)-\left(A^{\alpha} B^{\beta} K_{t}\right)(g ; h)\right| \\
& \quad \leq a t^{-\left(D^{\prime}+|\alpha|+|\beta|\right) / 2} e^{\omega t}\left(\frac{|k|^{\prime}+|l|^{\prime}}{t^{1 / 2}+\left|g h^{-1}\right|^{\prime}}\right)^{\nu} e^{-b\left(\left|g h^{-1}\right|^{\prime}\right)^{2} t^{-1}}
\end{aligned}
$$

for all $g, h, k, l \in G$ and $t>0$ with $|k|^{\prime}+|l|^{\prime} \leq \kappa t^{1 / 2}+2^{-1}\left|g h^{-1}\right|^{\prime}$. 
REMARK 1.2. Since the operators have complex coefficients there is $\theta_{C}>0$ such that $e^{i \phi} H$ is also subelliptic for $\phi \in\left\langle-\theta_{C}, \theta_{C}\right\rangle$. The estimates of Theorem 1.1 are then valid for $e^{i \phi} H$ instead of $H$. Moreover, for all $\theta \in\left\langle 0, \theta_{C}\right\rangle$ the constants in the kernel estimates are uniform for $\phi \in\langle-\theta, \theta\rangle$. Therefore one has estimates for the kernel $K_{z}$ similar to those of Theorem 1.1, if one replaces $t$ in the right hand sides by $|z|$, uniformly for all $z \in \mathbb{C} \backslash\{0\}$ with $|\arg z|<\theta$. All subsequent kernel estimates can be extended to complex time by this complex rotation.

This theorem demonstrates that Hölder continuity of the coefficients leads to differentiability of the kernel; there is a gain of one derivative simultaneously in each variable. This differentiability can be used to obtain links between the domain of powers of $H$ and the $C^{n}$-subspaces. The basic result of this nature is a Kato-type theorem characterizing the domain of the square root.

THEOREM 1.3. Let $H$ be a subelliptic second-order operator in divergence form (1). Suppose either

(a) $H$ is strongly elliptic, or

(b) $G$ is stratified and $a_{1}, \ldots, a_{d^{\prime}}$ is a basis for $\mathfrak{g}_{1}$ in the stratification $\left(\mathfrak{g}_{m}\right)_{m \in\{1, \ldots, r\}}$ of $\mathfrak{g}$.

If $c_{i j}, c_{i}, c_{i}^{\prime} \in C^{\nu \prime}$ for some $v \in\langle 0,1\rangle$ and $c_{0} \in L_{\infty}$ then $D\left((\lambda I+H)^{1 / 2}\right)=L_{p ; 1}^{\prime}$ for all large $\lambda>0$ and $p \in\langle 1, \infty\rangle$.

If $p=2$ then the strongly elliptic version of this result can be deduced from McIntosh's analysis of the Kato problem [McI]. McIntosh's $L_{2}$-result has been extended to the case $c_{i j} \in C^{\nu \prime}$ and $c_{i}, c_{i}^{\prime}, c_{0} \in L_{\infty}$ in [ElR5].

In order to obtain information about the domain of $H$ itself one needs more smoothness but one important input is a resolvent bound which only requires Hölder continuity of the principal coefficients. The following result is readily derived on $L_{2}$ and can be subsequently extended to the $L_{p}$-spaces with $p \in\langle 1, \infty\rangle$ by singular integration techniques based on the above kernel bounds.

THEOREM 1.4. Let $H$ be a subelliptic second-order operator in divergence form (1). Suppose either

(a) $H$ is strongly elliptic, or

(b) $G$ is stratified and $a_{1}, \ldots, a_{d^{\prime}}$ is a basis for $\mathfrak{g}_{1}$ in the stratification $\left(\mathfrak{g}_{m}\right)_{m \in\{1, \ldots, r)}$ of $\mathfrak{g}$.

If $c_{i j} \in C^{\nu}{ }^{\prime}(G)$ for some $v \in\langle 0,1\rangle$ and $c_{i}, c_{i}^{\prime}, c_{0} \in L_{\infty}$ then $A_{i}(I+H)^{-1} A_{j}$ extends to a bounded operator on $L_{p}$, or $L_{\hat{p}}$, for all $i, j \in\left\{1, \ldots, d^{\prime}\right\}$ and $p \in\langle 1, \infty\rangle$.

In order to convert the estimates of this theorem into information on the domain of $H$ one needs to be able to commute the derivatives $A_{j}$ to the left and this requires 
differentiability of the coefficients $c_{i j}$ and $c_{i}^{\prime}$. If, however, these coefficients are differentiable then $H$ can be written in non-divergence form,

$$
H=-\sum_{i, j=1}^{d} c_{i j} A_{i} A_{j}+\sum_{i=1}^{d} c_{i} A_{i}+c_{0} I .
$$

Not only is the domain result best described for operators of this form but the smoothness properties of the kernel can also be partially improved. Unfortunately, we can only prove the next results for strongly elliptic operators.

THEOREM 1.5. Let H be a second-order strongly elliptic operator in non-divergence form (4) with coefficients $c_{i j} \in L_{\infty ; 1}$ and $c_{i}, c_{0} \in L_{\infty}$.

I. If $c_{i j}, c_{i}, c_{0} \in L_{\infty ; n}$ with $n \in \mathbb{N}$ then $K_{t}$ is $(n+1)$-times left differentiable in the first variable and these derivatives are $(n-1)$-times left differentiable in the second variable. Moreover, for each $\nu \in\langle 0,1\rangle$ and $\kappa>0$ there exist $a, b>0$ and $\omega \geq 0$ such that

$$
\left|\left(A^{\alpha} B^{\beta} K_{t}\right)(g ; h)\right| \leq a t^{-d / 2} t^{-(|\alpha|+|\beta|) / 2} e^{\omega t} e^{-b\left|g h^{-1}\right|^{2} t^{-1}}
$$

and

$$
\begin{aligned}
& \left|\left(A^{\alpha} B^{\beta} K_{t}\right)\left(k^{-1} g ; l^{-1} h\right)-\left(A^{\alpha} B^{\beta} K_{t}\right)(g ; h)\right| \\
& \quad \leq a t^{-d / 2} t^{-(|\alpha|+|\beta|) / 2} e^{\omega t}\left(\frac{|k|+|l|}{t^{1 / 2}+\left|g h^{-1}\right|}\right)^{\nu} e^{-b\left|g h^{-1}\right|^{2} t^{-1}}
\end{aligned}
$$

uniformly for all $\alpha \in J_{n+1}(d), \beta \in J_{n-1}(d), t>0, g, h \in G$ and $k, l \in G$ such that $|k|+|l| \leq \kappa t^{1 / 2}+2^{-1}\left|g h^{-1}\right|$.

II. If $c_{i j}, c_{i}, c_{0} \in L_{\infty, n}(G)$ with $n \in \mathbb{N}$ and $\operatorname{Re} c_{0}$ large enough then $D\left(H^{m / 2}\right)=$ $L_{p ; m}$ for all $p \in(1, \infty)$ and $m \in\{1, \ldots, n+2\}$.

III. If $c_{i j}, c_{i}, c_{0} \in C^{n+v}(G)$ with $n \in \mathbb{N}$ and $0<\gamma<v<1$ then the kernel $K_{t}$ is $(n+2)$-times differentiable in the first variable, the derivatives with respect to the first variable are n-times differentiable with respect to the second, the derivatives are continuous,

$$
\left|\left(A^{\alpha} B^{\beta} K_{t}\right)(g ; h)\right| \leq a t^{-d / 2} t^{-(|\alpha|+|\beta|) / 2} e^{\omega t} e^{-b\left|g h^{-1}\right|^{2} t^{-1}}
$$

and

$$
\begin{aligned}
& \left|\left(A^{\alpha} B^{\beta} K_{t}\right)\left(k^{-1} g ; l^{-1} h\right)-\left(A^{\alpha} B^{\beta} K_{t}\right)(g ; h)\right| \\
& \quad \leq a t^{-d / 2} t^{-(|\alpha|+|\beta|) / 2} e^{\omega t}\left(\frac{|k|+|l|}{t^{1 / 2}+\left|g h^{-1}\right|}\right)^{\gamma} e^{-b\left|g h^{-1}\right|^{2} t^{-1}}
\end{aligned}
$$

uniformly for all $\alpha \in J_{n+2}(d), \beta \in J_{n}(d), t>0, g, h \in G$ and $k, l \in G$ such that $|k|+|l| \leq \kappa t^{1 / 2}+2^{-1}\left|g h^{-1}\right|$. 
In particular it follows that one has Gaussian estimates for all derivatives of the kernel if all the coefficients belong to $C_{b ; \infty}(G)$. In the situation of $m$ th order strongly elliptic operators on manifolds with bounded geometry this was proved by Kordyukov [Kor] using pseudo-differential operators.

\section{Preliminaries}

2.1. Spaces and embeddings For the convenience of the reader we first collect some definitions and inequalities which will be useful throughout the paper.

Define the norm $\|\cdot\|_{\infty}^{\Delta}: L_{1 . \text { loc }} \rightarrow[0, \infty]$ by $\|\varphi\|_{\infty}^{\Delta}=\left\|\Delta^{1 / 2} \varphi\right\|_{\infty}$ and for each $v \in\langle 0,1\rangle$ define $\|\cdot\| \|_{C_{\Delta}^{v}}: C(G) \rightarrow[0, \infty]$ by

$$
\|\varphi\|_{C_{\Delta}^{\nu^{\prime}}}=\sup _{e \neq g \in B^{\prime}(1)}\left(|g|^{\prime}\right)^{-v}\left\|\Delta^{1 / 2}(I-L(g)) \varphi\right\|_{\infty} .
$$

Then introduce the corresponding Banach spaces $L_{\infty}^{\Delta}=\left\{\varphi \in L_{1, \text { loc }}:\|\varphi\|_{\infty}^{\Delta}<\infty\right\}$ and $C_{\Delta}^{\nu \prime}=\left\{\varphi \in C(G):\|\varphi\|_{C_{\Delta}^{\prime \prime}}^{\prime}<\infty\right\}$.

One has the following continuity properties of multiplication operators.

LEMMA 2.1. Let $v \in\langle 0,1\rangle$.

I. If $\varphi, \psi \in C^{\nu \prime} \cap L_{\infty}$, then $\varphi \psi \in C^{\nu \prime}$ and

$$
\|\varphi \psi\|\left\|_{C^{v}} \leq\right\| \varphi\left\|_{C^{v^{\prime}}}\right\| \psi\left\|_{\infty}+\right\| \varphi\left\|_{\infty}\right\| \psi\|\|_{C^{\nu^{\prime}}} .
$$

II. If $\varphi \in C^{\nu \prime} \cap L_{\infty}$ and $\psi \in C_{\Delta}^{\nu \prime} \cap L_{\infty}^{\Delta}$, then $\varphi \psi \in C_{\Delta}^{\nu \prime}$ and

$$
\|\varphi \psi\|\left\|_{C_{\Delta}^{\prime \prime}} \leq\right\| \varphi\left\|_{C^{\prime}}\right\| \psi\left\|_{\infty}^{\Delta}+\right\| \varphi\left\|_{\infty}\right\| \psi\|\|_{C_{\Delta}^{\nu}} .
$$

Proof. For all $g \in G$ one has

$$
(I-L(g))(\varphi \psi)=\psi \cdot(I-L(g)) \varphi+(L(g) \varphi) \cdot(I-L(g)) \psi,
$$

from which the statements follow.

For a continuous function $\varphi$ define the right modulus of continuity, $\omega_{r}^{\prime}(\varphi ; \cdot):\langle 0, \infty\rangle$ $\rightarrow[0, \infty]$, by

$$
\omega_{r}^{\prime}(\varphi ; R)=\sup \left\{\|\left(I-L(h) \varphi \|_{\infty}: h \in B^{\prime}(R)\right\},\right.
$$

where $B^{\prime}(R)=B^{\prime}(e ; R)$. The function $\varphi$ is called right uniformly continuous if $\lim _{R \rightarrow 0} \omega_{r}^{\prime}(\varphi ; R)=0$. In particular, each element of $C^{\nu \prime}$ with $v>0$ is right uniformly continuous. 
If $\varphi \in L_{\infty ; 1}^{\prime}$ then

$$
\|(I-L(g)) \varphi\|_{\infty} \leq d^{\prime}|g|^{\prime} \max _{i \in\left\{1 \ldots, d^{\prime}\right\}}\left\|A_{i} \varphi\right\|_{\infty}, \quad \omega_{r}^{\prime}(\varphi ; R) \leq d^{\prime} R\|\varphi\|_{\infty ; 1}^{\prime}
$$

for all $g \in G$ and $R>0$. So $L_{\infty ; 1}^{\prime} \subset C^{\nu}$.

Recall the Sobolev spaces $L_{p ; n}^{\prime}, n \in \mathbb{N}_{0}$, are defined by $L_{p ; n}^{\prime}=\bigcap_{\alpha \in J_{n}\left(d^{\prime}\right)} D\left(A^{\alpha}\right)$ with norms $\|\varphi\|_{p ; n}^{\prime}=\sup _{\alpha \in J_{n}\left(d^{\prime}\right)}\left\|A^{\alpha} \varphi\right\|_{p}$. But for $p \in\langle 1, \infty\rangle$ one has

$$
L_{p ; n}^{\prime}=D\left(\left(I+H_{L}\right)^{n / 2}\right),
$$

where $H_{L}=-\sum_{i=1}^{d^{\prime}} A_{i}^{2}$ is the sublaplacian associated with the algebraic basis $a_{1}, \ldots, a_{d^{\prime}}$, and $\|\cdot\|_{p ; n}^{\prime}$ is equivalent to the norm $\varphi \mapsto\left\|\left(I+H_{L}\right)^{n / 2} \varphi\right\|_{p}$ (see [BER] and the appendix). Next, for $\gamma \in \mathbb{R} \backslash \mathbb{N}_{0}$ and $p \in\langle 1, \infty\rangle$ we define $L_{p ; \gamma}^{\prime}$ as the completion of $L_{p}$ with respect to the norm $\varphi \mapsto\left\|\left(I+H_{L}\right)^{\gamma / 2} \varphi\right\|_{p}$ (see also [EIR2, Section 3]). It then follows from the identity (7) that the $L_{p: \gamma}^{\prime}$ form a scale of complex interpolation spaces. Note also that for $n \in \mathbb{N}_{0}$ the space $L_{p ;-n}^{\prime}$ can be identified with the dual of $L_{p^{*} ; n}^{\prime}$ where $1 / p^{*}+1 / p=1$.

The scale of spaces $L_{\hat{p}: \gamma}^{\prime}, \gamma \in \mathbb{R}$, can be defined in a similar manner relative to the spaces $L_{\hat{p}}$ based on right Haar measure.

We adopt some conventions on the extension of operators. If $X$ is a bounded operator on $L_{2}$ such that $X\left(L_{2} \cap L_{p}\right) \subseteq L_{2} \cap L_{p}$ and $\|X \varphi\|_{p} \leq c_{p}\|\varphi\|_{p}$ for some $c_{p}>0$ and all $\varphi \in L_{2} \cap L_{p}$ then $X$ is norm densely-defined on $L_{p}$ and norm closable if $p \in[1, \infty)$. Hence $X$ extends by closure to a bounded operator on $L_{p}$. In this situation we abbreviate the description by saying that $X$ is bounded on $L_{p}$. More generally, if $X$ is a densely-defined, closable, operator from the Banach space $\mathscr{X}$ to the Banach space $\mathscr{Y}$ with bounded closure we say $X$ is a bounded operator from $\mathscr{X}$

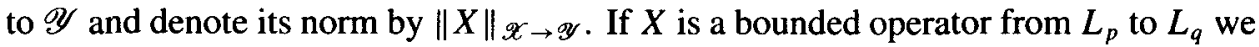
also use $\|X\|_{p \rightarrow q}$ to denote the norm.

Next we note some simple criteria for an operator to be bounded between the Sobolev spaces.

LEMMA 2.2. Let $p \in\langle 1, \infty\rangle$ and $n, m \in \mathbb{N}_{0}$. If $X$ is a bounded operator on $L_{p}$ then the following conditions are equivalent:

I. $X$ is bounded from $L_{p ;-n}^{\prime}$ to $L_{p ; m}^{\prime}$.

II. $X^{*}$ is bounded from $L_{p^{*} ;-m}^{\prime}$ to $L_{p^{*}: n}^{\prime}$, where $1 / p^{*}+1 / p=1$.

III. $\left(I+H_{L}\right)^{m / 2} X\left(I+H_{L}\right)^{n / 2}$ is bounded on $L_{p}$.

IV. $A^{\alpha} X A^{\beta}$ is bounded on $L_{p}$ for all $\alpha \in J_{m}\left(d^{\prime}\right)$ and $\beta \in J_{n}\left(d^{\prime}\right)$.

ProOF. The equivalence of I and II is by duality and the equivalence with III follows from [BER]. But then II is equivalent to boundedness of $\left(I+H_{L}\right)^{m / 2} X^{*}\left(I+H_{L}\right)^{n / 2}$ on $L_{p^{*}}$ and by [BER] this is equivalent to boundedness of the operators $A^{\beta} X^{*}\left(I+H_{L}\right)^{n / 2}$, 
$\beta \in J_{n}\left(d^{\prime}\right)$, on $L_{p^{*}}$ or boundedness of $\left(I+H_{L}\right)^{n / 2} X A^{\beta}, \beta \in J_{n}\left(d^{\prime}\right)$, on $L_{p}$. But this in turn is equivalent to 2.2 .

Note that if $X$ is bounded from $L_{p:-n}^{\prime}$ to $L_{p: m}^{\prime}$ the cross-norm of $X$ between the spaces can be chosen as

$$
\|X\|_{L_{p:-n}^{\prime} \rightarrow L_{p: m}^{\prime}}=\sup _{\alpha \in J_{m}\left(d^{\prime}\right), \beta \in J_{n}\left(d^{\prime}\right)}\left\|A^{\alpha} X A^{\beta}\right\|_{L_{p} \rightarrow L_{p}} .
$$

This is a direct consequence of the above arguments.

Each $\psi \in L_{\infty}$ is identified as a multiplication operator on $L_{p}, L_{p ; \gamma}^{\prime}$ or $L_{\hat{p}}$, etcetera and we next show that multiplication with a $C^{\nu} \cap \cap L_{\infty}$ function, $\nu \in\langle 0,1\rangle$, is a bounded operator on $L_{p: \gamma}^{\prime}$ for all $\gamma \in\langle 0, \nu\rangle$ and $p \in\langle 1, \infty\rangle$.

PROPOSITION 2.3. Let $v \in\langle 0,1\rangle$.

I. If $\gamma \in\langle 0, v\rangle$ and $p \in\langle 1, \infty\rangle$ then $\psi \varphi \in L_{p ; \gamma}^{\prime}$ for all $\psi \in C^{\nu \prime} \cap L_{\infty}$ and $\varphi \in L_{p: \gamma^{\prime}}^{\prime}$. Moreover, for all $\gamma \in\langle 0, \nu\rangle$ and $p \in\langle 1, \infty\rangle$ there exists a $c>0$ such that

$$
\|\psi \varphi\|_{p: \gamma}^{\prime} \leq c\left(\|\psi\|\left\|_{C^{\prime}}+\right\| \psi \|_{\infty}\right)\|\varphi\|_{p: \gamma}^{\prime}
$$

uniformly for all $\varphi \in L_{p ; \gamma}^{\prime}$ and $\psi \in C^{\nu \prime} \cap L_{\infty}$.

II. For all $n \in \mathbb{N}, \gamma \in\langle 0, v\rangle$ and $p \in\langle 1, \infty\rangle$ there exists a $c>0$ such that

$$
\|\psi \varphi\|_{p ; n+\gamma}^{\prime} \leq c\left(\sup _{\alpha \in J_{n}\left(d^{\prime}\right)}\left\|A^{\alpha} \psi\right\|_{C^{v}}+\|\psi\|_{\infty ; n}^{\prime}\right)\|\varphi\|_{p ; n+\gamma}^{\prime}
$$

uniformly for all $\varphi \in L_{p: n+\gamma}^{\prime}$ and $\psi \in C^{n+v}$.

III. There exists $\lambda_{0}>0$ such that for all $\gamma \in\langle 0, v\rangle$ there is ac $>0$ such that

$$
\left\|\left[\left(\lambda I+H_{L}\right)^{\gamma / 2}, \psi\right] \varphi\right\|_{p} \leq c \lambda^{-(\nu-\gamma) / 2}\left(\|\mid \psi\|_{C^{\nu}}+\|\psi\|_{\infty}\right)\|\varphi\|_{p}
$$

uniformly for $\lambda \geq \lambda_{0}, p \in(1, \infty), \psi \in C^{\nu \prime} \cap L_{\infty}$ and $\varphi \in L_{p ; \gamma}^{\prime}$.

IV. If $d^{\prime}=d$ then there exists $\lambda_{0}>0$ such that for all $p \in\langle 1, \infty\rangle, \gamma \in\langle 0, v\rangle$ and $\lambda \geq \lambda_{0}$ there is $a c>0$ such that

$$
\left\|\left[\left(\lambda I+H_{L}\right)^{(1+\gamma) / 2}, \psi\right] \varphi\right\|_{p} \leq c\left(\sup _{i \in\{1, \ldots, d\}}\left\|A_{i} \psi\right\|_{C^{v}}+\|\psi\|_{C^{v}}+\|\psi\|_{\infty}\right)\|\varphi\|_{p ; 1}
$$

uniformly for all $\varphi \in L_{p ; 1+\gamma}$ and $\psi \in C^{1+\nu}$.

Similar statements are valid on the $L_{\hat{p}}$-spaces.

PROOF. We first prove a weaker version of Statement III, since we only know that a multiplication operator is bounded on $L_{p}$. Let $S$ and $K$ be the semigroup generated by $H_{L}$ and its kernel. There exist $a, b, \omega>0$ such that $\left|K_{t}(g)\right| \leq a t^{-D^{\prime} / 2} e^{-b\left(|g|^{\prime}\right)^{2} t^{-1}} e^{\omega t}$ 
for all $t>0$ and $g \in G$. Set $c_{\gamma}=\int_{0}^{\infty} d t t^{-1-\gamma / 2}\left(1-e^{t}\right)$. Then for all $\lambda>0$ and $\varphi \in L_{p ; 1}^{\prime}$ one has

$$
\left(\lambda I+H_{L}\right)^{\gamma / 2} \varphi=c_{\gamma}^{-1} \int_{0}^{\infty} d t t^{-1-\gamma / 2}\left(I-e^{-\lambda t} S_{t}\right) \varphi
$$

So if $\eta \in L_{q ; \gamma}^{\prime}$ with $1 / p+1 / q=1$ then

$$
\begin{aligned}
((\lambda I & \left.\left.+H_{L}\right)^{\gamma / 2} \eta, \psi \varphi\right)-\left(\eta, \psi\left(\lambda I+H_{L}\right)^{\gamma / 2} \varphi\right) \\
& =-c_{\gamma}^{-1} \int_{0}^{\infty} d t t^{-1-\gamma / 2}\left(\eta,\left[e^{-\lambda t} S_{t}, \psi\right] \varphi\right) \\
& =c_{\gamma}^{-1} \int_{0}^{\infty} d t t^{-1-\gamma / 2} \int_{G} d g K_{t}(g) e^{-\lambda t}(\eta,[I-L(g), \psi] \varphi) \\
& =c_{\gamma}^{-1} \int_{0}^{\infty} d t t^{-1-\gamma / 2} \int_{G} d g K_{t}(g) e^{-\lambda t}(\eta,(I-L(g)) \psi \cdot L(g) \varphi),
\end{aligned}
$$

where we used (5). Since $\psi \in C^{\nu \prime} \cap L_{\infty}$ one has $\|(I-L(g)) \psi\|_{\infty} \leq c_{\psi}\left(|g|^{\prime}\right)^{\nu}$ for all $g \in G$, where $c_{\psi}=\|\| \psi\left\|_{C^{\nu}}+2\right\| \psi \|_{\infty}$. Then

$$
\begin{aligned}
\mid((\lambda I & \left.\left.+H_{L}\right)^{\gamma / 2} \eta, \psi \varphi\right)-\left(\eta, \psi\left(\lambda I+H_{L}\right)^{\gamma / 2} \varphi\right) \mid \\
& \leq a c_{\psi} c_{\gamma}^{-1} \int_{0}^{\infty} d t t^{-1+(\nu-\gamma) / 2} \int_{G} d g t^{-D^{\prime} / 2} e^{-b\left(|g|^{\prime}\right)^{2} t^{-1}} e^{-(\lambda-\omega) t}\left(\left(|g|^{\prime}\right)^{2} t^{-1}\right)^{\nu / 2}\|\varphi\|_{p}\|\eta\|_{q} \\
& \leq a^{\prime} c_{\psi} \int_{0}^{\infty} d t t^{-1+(\nu-\gamma) / 2} \int_{G} d g t^{-D^{\prime} / 2} e^{-b^{\prime}\left(|g|^{\prime}\right)^{2} t^{-1}} e^{-(\lambda-\omega) t}\|\varphi\|_{p}\|\eta\|_{q} \\
& \leq a^{\prime \prime} c_{\psi} \int_{0}^{\infty} d t t^{-1+(\nu-\gamma) / 2} e^{-\left(\lambda-\omega^{\prime}\right) t}\|\varphi\|_{p}\|\eta\|_{q} \\
& \leq a^{\prime \prime \prime} c_{\psi}\left(\lambda-\omega^{\prime}\right)^{-(\nu-\gamma) / 2}\|\varphi\|_{p}\|\eta\|_{q}
\end{aligned}
$$

for some constants $a^{\prime}, a^{\prime \prime}, a^{\prime \prime \prime}, b^{\prime}, \omega^{\prime}>0$, independent of $\lambda, \psi, \varphi, \eta$ and $p$ uniformly for all $\lambda \geq \lambda_{0}=2 \omega^{\prime}$. Since $L_{p ; 1}^{\prime}$, and $L_{q ; 1}^{\prime}$, is a core for $\left(\lambda I+H_{L}\right)^{\gamma / 2}$ it follows that

$$
\begin{array}{r}
\left|\left(\left(\lambda I+H_{L}\right)^{\gamma / 2} \eta, \psi \varphi\right)-\left(\eta, \psi\left(\lambda I+H_{L}\right)^{\gamma / 2} \varphi\right)\right| \\
\leq a^{\prime \prime \prime} c_{\psi}\left(\lambda-\omega^{\prime}\right)^{-(\nu-\gamma) / 2}\|\varphi\|_{p}\|\eta\|_{q}
\end{array}
$$

for all $\varphi \in D\left(\left(\lambda I+H_{L}\right)^{\gamma / 2}\right)=L_{p ; \gamma}^{\prime}$ and $\eta \in D\left(\left(\lambda I+H_{L}\right)^{\gamma / 2}\right)=L_{q: \gamma}^{\prime}$.

Next, for all $\varphi \in L_{p ; \gamma}^{\prime}$ and $\lambda=\lambda_{0}$ one has

$$
\begin{aligned}
& \left|\left(\left(\lambda I+H_{L}\right)^{\gamma / 2} \eta, \psi \varphi\right)\right| \\
& \quad \leq c\left(\|\psi\|\left\|_{C^{\prime}}+\right\| \psi \|_{\infty}\right)\|\varphi\|_{p}\|\eta\|_{q}+\|\psi\|_{\infty}\left\|\left(\lambda I+H_{L}\right)^{\gamma / 2} \varphi\right\|_{p}\|\eta\|_{q} \\
& \quad \leq c^{\prime}\left(\|\psi\|\left\|_{C^{\nu}}+\right\| \psi \|_{\infty}\right)\left\|\left(\lambda I+H_{L}\right)^{\gamma / 2} \varphi\right\|_{p}\|\eta\|_{q}
\end{aligned}
$$


for all $\eta \in D\left(\left(\lambda I+H_{L}\right)^{\gamma / 2}\right)=L_{q ; \gamma}^{\prime}$. Therefore $\psi \varphi \in D\left(\left(\left(\lambda I+H_{L}\right)^{\gamma / 2}\right)^{*}\right)=L_{p ; \gamma}^{\prime}$. Statement I follows since the norms $\varphi \mapsto\left\|\left(\lambda I+H_{L}\right)^{\gamma / 2} \varphi\right\|_{p}$ and $\|\cdot\|_{p: \gamma}^{\prime}$ are equivalent (see [Rob, Lemma II.3.2]). Then Statement III is a consequence of (9) and a density argument.

For the proof of Statement II, let $\varphi \in L_{p ; n+\gamma}^{\prime}$ and $\psi \in C^{n+\nu}$. Then for all $\alpha \in J_{n}\left(d^{\prime}\right)$ the function $A^{\alpha}(\psi \varphi)$ can be written as a finite linear combination of terms of the form $\left(A^{\alpha_{1}} \varphi\right)\left(A^{\alpha_{2}} \psi\right)$ with $\alpha_{1}, \alpha_{2} \in J_{n}\left(d^{\prime}\right)$, by the Leibniz rule. But $A^{\alpha_{1}} \varphi \in L_{p ; \gamma}^{\prime}$ and $A^{\alpha_{2}} \psi \in C^{\nu \prime}$ and hence $\left(A^{\alpha_{1}} \varphi\right)\left(A^{\alpha_{2}} \psi\right) \in L_{p ; \gamma}^{\prime}$ by Statement I. So $A^{\alpha}(\psi \varphi) \in L_{p ; \gamma}^{\prime}$ and $\psi \varphi \in L_{p ; n+\gamma}^{\prime}$. The bounds follow from the proof.

Finally we prove Statement IV. Set $\tau=2^{-1}(1+\gamma)$. Then

$$
\begin{aligned}
\|[(\lambda I & \left.\left.+H_{L}\right)^{(1+\gamma) / 2}, \psi\right] \varphi \|_{p} \\
& \leq\left\|\left(\lambda I+H_{L}\right)^{\tau / 2}\left[\left(\lambda I+H_{L}\right)^{\tau / 2}, \psi\right] \varphi\right\|_{p}+\left\|\left[\left(\lambda I+H_{L}\right)^{\tau / 2}, \psi\right]\left(\lambda I+H_{L}\right)^{\tau / 2} \varphi\right\|_{p} \\
& \leq c\left\|\left[\left(\lambda I+H_{L}\right)^{\tau / 2}, \psi\right] \varphi\right\|_{p ; 1}+c\left(\|\psi\|_{C^{\nu}}+\|\psi\|_{\infty}\right)\left\|\left(\lambda I+H_{L}\right)^{\tau / 2} \varphi\right\|_{p} \\
& \leq c\left\|\left[\left(\lambda I+H_{L}\right)^{\tau / 2}, \psi\right] \varphi\right\|_{p ; 1}+c\left(\|\psi \psi\|_{C^{\nu}}+\|\psi\|_{\infty}\right)\|\varphi\|_{p ; 1}
\end{aligned}
$$

where we have used Statement III. For an estimate on the first term we argue as in (8), with $\gamma$ replaced by $\tau$. Now $\|(I-L(g)) \psi \cdot L(g) \varphi\|_{p ; 1} \leq 2 \|(I-$ $L(g)) \psi\left\|_{\infty ; 1}\right\| L(g) \varphi \|_{p ; 1}$. But $C^{1+\nu}=\left(L_{\infty}, L_{\infty ; 1}\right)_{\nu, \infty ; K ; 1}=\left(L_{\infty ; 1}, L_{\infty ; 2}\right)_{v, \infty ; K}$ by [ElR1, Theorem 2.1]. Therefore $\|(I-L(g)) \psi\|_{\infty ; 1} \leq c c_{\psi}^{\prime}|g|^{v} e^{\omega|g|}$ for all $g \in G$, where $c_{\psi}^{\prime}=\sup _{i \in\{1, \ldots, d\}}\left\|A_{i} \psi\right\|_{C^{v}}+\|\psi\|_{C^{v}}+\|\psi\|_{\infty}$. Moreover, $\|L(g) \varphi\|_{p: 1} \leq c^{\prime} e^{\omega^{\prime}|g|}\|\varphi\|_{p: 1}$. So $\|(I-L(g)) \psi \cdot L(g) \varphi\|_{p ; 1} \leq c c^{\prime} c_{\psi}^{\prime}|g|^{\nu} e^{\left(\omega+\omega^{\prime}\right)|g|}$ and one can continue to argue as in the final part of the proof of Statement III.

2.2. De Giorgi estimates The aim of this subsection is to prove the De Giorgi estimates involving derivatives which are crucial for the regularity theorem in the next section. Proposition 2.6 establishes a De Giorgi inequality for operators with $C^{v}$-coefficients but the basic difficulty is to prove the inequality for operators with constant coefficients. For these estimates we need the following space

$$
H_{2 ; 1}^{\prime}\left(B^{\prime}(g ; r)\right)=\left\{\varphi \in L_{2}\left(B^{\prime}(g ; r)\right): A_{i} \varphi \in L_{2}\left(B^{\prime}(g ; r)\right) \quad \text { for all } i \in\left\{1, \ldots, d^{\prime}\right\}\right\} \text {, }
$$

where $g \in G, r>0$ and $A_{i} \varphi$ denotes the distributional derivative in $\mathscr{D}^{\prime}\left(B^{\prime}(g ; r)\right)$. These spaces are equipped with the norms $\varphi \mapsto\left(\|\varphi\|_{2, g, r}^{2}+\left\|\nabla^{\prime} \varphi\right\|_{2, g, r}^{2}\right)^{1 / 2}$ where $\|\varphi\|_{2, g, r}=\left(\int_{B^{\prime}(g ; r)} d h|\varphi(h)|^{2}\right)^{1 / 2}$ and

$$
\left\|\nabla^{\prime} \varphi\right\|_{2, g, r}=\left(\int_{B^{\prime}(g ; r)} d h \sum_{i=1}^{d^{\prime}}\left|\left(A_{i} \varphi\right)(h)\right|^{2}\right)^{1 / 2} .
$$

We denote by $\stackrel{\circ}{H}_{2 ; 1}^{\prime}\left(B^{\prime}(g ; r)\right)$ the closure of $C_{c}^{\infty}\left(B^{\prime}(g ; r)\right)$ in $H_{2 ; 1}^{\prime}\left(B^{\prime}(g ; r)\right)$. 
For $\varphi \in L_{1, \text { loc }}$ we denote by $\langle\varphi\rangle_{g, r}$ the average of $\varphi$ over $B^{\prime}(g ; r)$. If $g=e$ we drop the $e$ in the notation. So $B^{\prime}(r)=B^{\prime}(e ; r),\left\|\nabla^{\prime} \varphi\right\|_{2, r}=\left\|\nabla^{\prime} \varphi\right\|_{2, e, r}$ etcetera.

The proofs of the De Giorgi estimates for strongly elliptic operators and for subelliptic operators on stratified groups are quite distinct.

2.2.1. Strongly elliptic operators with constant coefficients The exponent $d+2$ in the term $(r / R)^{d+2}$ in the next lemma is slightly better than we need for the regularity theorem in the next section. In fact, we only need $(r / R)^{d+2 v}$ for all $v \in\langle 0,1\rangle$, as in [ElR6, Section 3]. On the other hand, we now have an additional error term.

LEMMA 2.4. Let $H=-\sum_{i, j=1}^{d} c_{i j} A_{i} A_{j}$ be a pure second-order strongly elliptic operator with constant coefficients.

Then there exist $c_{D G}, c_{D G}^{\prime}>0$ such that for all $R \in\langle 0,1]$, all $g \in G$ and all $\varphi \in H_{2 ; 1}(B(g ; R))$ satisfying $H \varphi=0$ weakly on $B(g ; R)$ one has

$$
\begin{aligned}
& \sum_{k=1}^{d} \int_{B(g ; r)}\left|A_{k} \varphi-\left\langle A_{k} \varphi\right\rangle_{g, r}\right|^{2} \\
& \quad \leq c_{D G}(r / R)^{d+2} \sum_{k=1}^{d} \int_{B(g ; R)}\left|A_{k} \varphi-\left\langle A_{k} \varphi\right\rangle_{g, R}\right|^{2}+c_{D G}^{\prime} R^{2} \int_{B(g ; R)}|\nabla \varphi|^{2}
\end{aligned}
$$

for all $0<r \leq R$. Moreover, the constants $c_{D G}$ and $c_{D G}^{\prime}$ depend on the coefficients of $H$ only through $\mu_{C}$ and $\|C\|_{\infty}$.

PROOF. Arguing as in the proof of [ElR6, Proposition 3.4] it suffices to consider $g=e$.

Let $\|\cdot\|$ be the Euclidean norm on $\mathfrak{g}$ with respect to the basis $a_{1}, \ldots, a_{d}$. For all $s>0$ set $\widetilde{B}(s)=\{a \in \mathfrak{g}:\|a\|<s\}$. There exist $R_{0} \in\langle 0,1], c>1$ and a real analytic function $\sigma: \widetilde{B}\left(R_{0}\right) \rightarrow\left[c^{-1}, c\right]$ such that exp is a diffeomorphism from $\widetilde{B}\left(2 R_{0}\right)$ onto $\exp \widetilde{B}\left(2 R_{0}\right), c^{-1}\|a\| \leq|\exp a| \leq c\|a\|$ for all $a \in \mathfrak{g}$ with $\|a\| \leq R_{0}$,

$$
\int_{\tilde{B}\left(R_{0}\right)} d a \sigma(a) \varphi(\exp (a))=\int_{G} d g \varphi(g)
$$

for all $\varphi \in C_{c}^{\infty}\left(\exp \widetilde{B}\left(R_{0}\right)\right)$ and $\sigma(0)=1$. Then $B_{c^{-1} s} \subseteq \exp \widetilde{B}(s) \subseteq B_{c s}$ for all $s \in\left\langle 0, c^{-1} R_{0}\right]$. Let $D_{i}$ be the closed operator on $L_{2}(g)$ of left differentiation in the $i$ th direction. So

$$
\left(D_{i} \psi\right)(a)=\left.\frac{d}{d t} \psi\left(a-t a_{i}\right)\right|_{t=0} .
$$

Define the operator $\widetilde{A}_{i}$ on $\widetilde{B}\left(R_{0}\right)$ by $\widetilde{A}_{i}(\varphi \circ \exp )=\left(A_{i} \varphi\right) \circ \exp$. Then there exist real analytic functions $b_{i j}$ on $\widetilde{B}\left(2 R_{0}\right)$ such that $D_{i}=\tilde{A}_{i}+\sum_{j=1}^{d} b_{i j} D_{j}$ and $b_{i j}(0)=0$.

We compare the operator $H$ on $G$ with the operator $\widetilde{H}=-\sum c_{i j} D_{i} D_{j}$ on the commutative group g. By [Gial, Theorem III.2.1], applied to $D_{k} \psi$, there exists a 
constant $c_{D G}>0$, depending on the coefficients only through $\mu_{C}$ and $\|C\|_{\infty}$, such that for all $R \in\langle 0,1]$ and all $\psi \in H_{2: 1}(\widetilde{B}(R))$ satisfying $\widetilde{H} \psi=0$ weakly on $\widetilde{B}(R)$ one has

$$
\sum_{k=1}^{d} \int_{\widetilde{B}(r)}\left|D_{k} \psi-\left\langle D_{k} \psi\right\rangle_{\tilde{B}(r)}\right|^{2} \leq c_{D G}(r / R)^{d+2} \sum_{k=1}^{d} \int_{\widetilde{B}(R)}\left|D_{k} \psi-\left\langle D_{k} \psi\right\rangle_{\widetilde{B}(R)}\right|^{2}
$$

for all $0<r \leq R$, where $\langle\varphi\rangle_{\widetilde{B}(r)}$ denotes the average over the ball $\widetilde{B}(r)$.

Now let $R \in\left\langle 0, R_{0}\right], \varphi \in H_{2 ; 1}(B(R))$ and suppose that $H \varphi=0$ weakly on $B(R)$. Set $R_{1}=c^{-1} R$. Define $\tilde{\varphi} \in H_{2 ; 1}\left(\widetilde{B}\left(R_{1}\right)\right)$ by $\tilde{\varphi}=\varphi \circ \exp$ and let $\eta \in H_{2 ; 1}\left(\widetilde{B}\left(R_{1}\right)\right)$ be such that

$$
\widetilde{H} \eta=0 \text { weakly in } \widetilde{B}\left(R_{1}\right), \quad \chi=\tilde{\varphi}-\eta \in \stackrel{\circ}{H}_{2: 1}\left(\widetilde{B}\left(R_{1}\right)\right) \text {. }
$$

Then, since $\gamma \mapsto \int_{B(r)}|\psi-\gamma|^{2}$ attains its minimum at $\gamma=\langle\psi\rangle_{r}$, one has

$$
\begin{aligned}
\int_{\tilde{B}(r)} & \left|D_{k} \tilde{\varphi}-\left\langle D_{k} \tilde{\varphi}\right\rangle_{\tilde{B}(r)}\right|^{2} \\
& \leq \int_{\widetilde{B}(r)}\left|D_{k} \tilde{\varphi}-\left\langle D_{k} \eta\right\rangle_{\tilde{B}(r)}\right|^{2} \\
& \leq 2 \int_{\tilde{B}(r)}\left|D_{k} \eta-\left\langle D_{k} \eta\right\rangle_{\tilde{B}(r)}\right|^{2}+2 \int_{\tilde{B}(r)}\left|D_{k} \chi\right|^{2} \\
& \leq 2 c_{D G}\left(r / R_{1}\right)^{d+2} \int_{\widetilde{B}\left(R_{1}\right)}\left|D_{k} \eta-\left\langle D_{k} \eta\right\rangle_{\tilde{B}\left(R_{1}\right)}\right|^{2}+2 \int_{\tilde{B}\left(R_{1}\right)}\left|D_{k} \chi\right|^{2} \\
& \leq 2 c_{D G}\left(r / R_{1}\right)^{d+2} \int_{\widetilde{B}\left(R_{1}\right)}\left|D_{k} \eta-\left\langle D_{k} \tilde{\varphi}\right\rangle_{\tilde{B}\left(R_{1}\right)}\right|^{2}+2 \int_{\tilde{B}\left(R_{1}\right)}\left|D_{k} \chi\right|^{2} \\
& \leq 4 c_{D G}\left(r / R_{1}\right)^{d+2} \int_{\widetilde{B}\left(R_{1}\right)}\left|D_{k} \tilde{\varphi}-\left\langle D_{k} \tilde{\varphi}\right\rangle_{\tilde{B}\left(R_{1}\right)}\right|^{2}+\left(2+4 c_{D G}\right) \int_{\widetilde{B}\left(R_{1}\right)}\left|D_{k} \chi\right|^{2}
\end{aligned}
$$

for all $0<r \leq R_{1}$ and $k \in\{1, \ldots, d\}$.

Next introduce $\hat{\chi} \in \stackrel{\circ}{H}_{2 ; 1}(B(R))$ such that $\chi=\hat{\chi} \circ \exp$. Then

$$
0=(\hat{\chi}, H \varphi)=\sum_{i, j} \int_{G} c_{i j} \overline{A_{i} \hat{\chi}} A_{j} \varphi=\sum_{i, j} \int_{\widetilde{B}\left(R_{t}\right)} \sigma c_{i j} \overline{\widetilde{A}_{i} \chi} \widetilde{A_{j}} \tilde{\varphi}
$$

Therefore

$$
\begin{aligned}
u_{C} \sum_{k} \int_{\tilde{B}\left(R_{1}\right)}\left|D_{k} \chi\right|^{2} & \leq \operatorname{Re}(\chi, \widetilde{H} \chi)=\operatorname{Re}(\chi, \widetilde{H} \tilde{\varphi})=\operatorname{Re} \sum_{i, j} \int_{\widetilde{B}\left(R_{1}\right)} c_{i j} \overline{D_{i} \chi} D_{j} \tilde{\varphi} \\
& =\operatorname{Re} \sum_{i, j} c_{i j} \int_{\widetilde{B}\left(R_{1}\right)}\left(\overline{D_{i} \chi} D_{j} \tilde{\varphi}-\sigma \widetilde{\widetilde{A}_{i} \chi} \widetilde{A_{j}} \tilde{\varphi}\right) .
\end{aligned}
$$


Since the functions $\sigma$ and $b_{i j}$ are real analytic, $\sigma(0)=1$ and $b_{i j}(0)=0$, one easily estimates that

$$
\left|\int_{\widetilde{B}\left(R_{1}\right)} \overline{D_{i} \chi} D_{j} \tilde{\varphi}-\sigma \overline{A_{i} \chi} \widetilde{A}_{j} \tilde{\varphi}\right| \leq c^{\prime} R\left(\sum_{i} \int_{\widetilde{B}\left(R_{1}\right)}\left|D_{i} \chi\right|^{2}\right)^{1 / 2}\left(\sum_{i} \int_{B(R)}\left|A_{i} \varphi\right|^{2}\right)^{1 / 2}
$$

for some $c^{\prime}>0$ which depends only on $G$ and the basis $a_{1}, \ldots, a_{d}$. Hence

$$
\sum_{k} \int_{\widetilde{B}\left(R_{1}\right)}\left|D_{k} \chi\right|^{2} \leq\left(c^{\prime} d^{2}\|C\|_{\infty} \mu_{C}^{-1}\right)^{2} \int_{B(R)} R^{2}|\nabla \varphi|^{2}
$$

So

$$
\begin{aligned}
\sum_{k} \int_{\widetilde{B}(r)}\left|D_{k} \tilde{\varphi}-\left\langle D_{k} \tilde{\varphi}\right\rangle_{\tilde{B}(r)}\right|^{2} \leq & 4 c_{D G}\left(r / R_{1}\right)^{d+2} \int_{\tilde{B}\left(R_{1}\right)}\left|D_{k} \tilde{\varphi}-\left\langle D_{k} \tilde{\varphi}\right\rangle_{\tilde{B}\left(R_{1}\right)}\right|^{2} \\
& +\left(2+4 c_{D G}\right)\left(c^{\prime} d^{2}\|C\|_{\infty} \mu_{C}^{-1}\right)^{2} R^{2} \int_{B(R)}|\nabla \varphi|^{2}
\end{aligned}
$$

for all $0<r \leq R_{1}$. Again using the real analytic functions $\sigma$ and $b_{i j}$ one deduces as above that there exist a $c^{\prime \prime}>0$, depending only on $G$ and the basis $a_{1}, \ldots, a_{d}$, such that

$$
\begin{aligned}
\int_{\widetilde{B}(r)}\left|D_{k} \tilde{\varphi}-\left\langle D_{k} \tilde{\varphi}\right\rangle_{\tilde{B}(r)}\right|^{2} & \leq \int_{\widetilde{B}(r)}\left|D_{k} \tilde{\varphi}-\left\langle A_{k} \varphi\right\rangle_{c r}\right|^{2} \\
& \leq 2 \int_{\tilde{B}(r)}\left|\tilde{A}_{k} \tilde{\varphi}-\left\langle A_{k} \varphi\right\rangle_{c r}\right|^{2}+2 \int_{\widetilde{B}(r)}\left|\sum_{j} b_{k j} D_{j} \tilde{\varphi}\right|^{2} \\
& \leq 2 c \int_{B(c r)}\left|A_{k} \varphi-\left\langle A_{k} \varphi\right\rangle_{c r}\right|^{2}+c^{\prime \prime} r^{2} \int_{B(c r)}|\nabla \varphi|^{2}
\end{aligned}
$$

and

$$
\int_{B(r)}\left|A_{k} \varphi-\left\langle A_{k} \varphi\right\rangle_{r}\right|^{2} \leq 2 c \int_{\widetilde{B}(c r)}\left|D_{k} \tilde{\varphi}-\left\langle D_{k} \tilde{\varphi}\right\rangle_{\widetilde{B}(c r)}\right|^{2}+c^{\prime \prime} r^{2} \int_{B(c r)}|\nabla \varphi|^{2}
$$

for all $r \in\left\langle 0, R_{1}\right]$. Combining these estimates it follows that

$$
\begin{gathered}
\sum_{k=1}^{d} \int_{B(r)}\left|A_{k} \varphi-\left\langle A_{k} \varphi\right\rangle_{r}\right|^{2} \leq 16 c^{d+4} c_{D G}\left(r / R_{1}\right)^{d+2} \sum_{k=1}^{d} \int_{B(R)}\left|A_{k} \varphi-\left\langle A_{k} \varphi\right\rangle_{R}\right|^{2} \\
+c_{D G}^{\prime} R^{2} \int_{B(R)}|\nabla \varphi|^{2}
\end{gathered}
$$

for some $c_{D G}^{\prime}>0$, uniformly for all $0<r \leq c^{-1} R$ and $R \leq R_{0}$. One can now extend the estimates to the range $0<r \leq R \leq 1$ by the argument at the end of the proof of [ElR6, Proposition 3.4]. 
2.2.2. Subelliptic operators on stratified groups with constant coefficients Let $G$ be a (connected, simply connected) stratified Lie group and $a_{1}, \ldots, a_{d^{\prime}}$ a basis for $\mathfrak{g}_{1}$ in the stratification $\left(\mathfrak{g}_{n}\right)_{n \in \mathfrak{i} 1 \ldots ., r}$ of $\mathfrak{g}$.

LEMMA 2.5. Let $H=-\sum_{i, j=1}^{d^{\prime}} c_{i j} A_{i} A_{j}$ be a pure second-order subelliptic operator with constant coefficients. Then for all $\nu \in\langle 0,1\rangle$ there exists a $c_{D G}>0$ such that for all $R \in\langle 0,1], g \in G$ and $\varphi \in H_{2 ; 1}^{\prime}\left(B^{\prime}(g ; R)\right)$ satisfying $H \varphi=0$ weakly on $B^{\prime}(g ; R)$ one has

$$
\sum_{k=1}^{d} \int_{B^{\prime}(g ; r)}\left|A_{k} \varphi-\left\langle A_{k} \varphi\right\rangle_{g, r}\right|^{2} \leq c_{D G}(r / R)^{D^{\prime}+2 v} \sum_{k=1}^{d} \int_{B^{\prime}(g ; R)}\left|A_{k} \varphi-\left\langle A_{k} \varphi\right\rangle_{g, R}\right|^{2}
$$

for all $0<r \leq R$. Moreover, $c_{D G}$ depends on the coefficients of $H$ only through $\mu_{C}$ and $\|C\|_{\infty}$.

ProOF. Again it suffices to consider $g=e$. Since $G$ is nilpotent, connected and simply connected it follows that the exponential map is an analytic diffeomorphism from $\mathfrak{g}$ onto $G$. Extend the algebraic basis $a_{1}, \ldots, a_{d^{\prime}}$ to a basis $a_{1}, \ldots, a_{d}$ for $\mathfrak{g}$ such that $a_{i} \in \mathfrak{g}_{n}$ for some $n$, for all $i \in\{1, \ldots, d\}$. For all $i \in\left\{1, \ldots, d^{\prime}\right\}$ define $\chi_{i}: G \rightarrow \mathbb{R}$ by $\chi_{i}\left(\sum_{j=1}^{d} \xi_{j} a_{j}\right)=\xi_{i}$ for all $\xi \in \mathbb{R}^{d}$. Then $\chi_{i}$ is a $C^{\infty}$-function and $A_{j} \chi_{i}=-\delta_{i j}$ for all $i, j \in\left\{1, \ldots, d^{\prime}\right\}$, since $\mathfrak{g}$ is stratified. Hence $A_{k} A_{j} \chi_{i}=0$ for all $i, j, k \in\left\{1, \ldots, d^{\prime}\right\}$.

Now the proof of this lemma is a modification of the proof of [EIR6, Proposition 3.3]. We use the notation of [EIR6]. In particular, let $R_{0} \in\langle 0,1], \sigma \in\langle 0,1\rangle$ and let the cut-off functions $\eta_{R}$ be as in [ElR6, Lemma 2.3].

Let $R \in\left\langle 0, R_{0}\right.$ ], $\varphi \in H_{2 ; 1}^{\prime}\left(B^{\prime}(R)\right)$ and assume $H \varphi=0$ weakly on $B^{\prime}(R)$. For all $i \in\left\{1, \ldots, d^{\prime}\right\}$ set $b_{i}=\left\langle A_{i} \varphi\right\rangle_{R}, \psi=\varphi+\sum_{i=1}^{d^{\prime}} b_{i} \chi_{i}$ and $\tau=\psi-\langle\psi\rangle_{R}$. Then $\langle\tau\rangle_{R}=0, A_{i} \tau=A_{i} \varphi-b_{i}$ and hence $\left\langle A_{i} \tau\right\rangle_{R}=0$ for all $i \in\left\{1, \ldots, d^{\prime}\right\}$. Moreover, $H \tau=H \varphi=0$, since the second-order derivatives of the $\chi_{i}$ vanish. Hence it follows as in the proof of [EIR6, Proposition 3.3], with $\varphi$ replaced by $A_{k} \tau$, that for all $r \leq \sigma R$ one has

$$
\begin{aligned}
\left(\sum_{k=1}^{d^{\prime}} \int_{B^{\prime}\left(\sigma^{-1} r\right)}\left|A_{k} \varphi-\left\langle A_{k} \varphi\right\rangle_{\sigma^{-1} r}\right|^{2}\right)^{1 / 2} & \leq\left(\sum_{k=1}^{d^{\prime}} \int_{B^{\prime}\left(\sigma^{-1} r\right)}\left|A_{k} \tau-\left\langle A_{k} \tau\right\rangle_{\sigma^{-1} r}\right|^{2}\right)^{1 / 2} \\
& \leq c^{\prime} r^{D^{\prime} / 2+\nu} \sum_{k=1}^{d^{\prime}}\left\|\eta_{R} A_{k} \tau\right\|_{C^{\prime \prime}}
\end{aligned}
$$

But

$$
\begin{aligned}
\left\|\eta_{R} A_{k} \tau\right\|_{c^{\nu}} & \leq \varepsilon^{n-D^{\prime} / 2-\nu} N_{2 ; n}^{\prime}\left(\eta_{R} A_{k} \tau\right)+c \varepsilon^{-D^{\prime} / 2-v}\left(\int_{B^{\prime}(R)}\left|A_{k} \tau\right|^{2}\right)^{1 / 2} \\
& =R^{n-D^{\prime} / 2-\nu} N_{2 ; n}^{\prime}\left(\eta_{R} A_{k} \tau\right)+c R^{-D^{\prime} / 2-\nu}\left(\int_{B^{\prime}(R)}\left|A_{k} \varphi-\left\langle A_{k} \varphi\right\rangle_{R}\right|^{2}\right)^{1 / 2}
\end{aligned}
$$


where we have set $\varepsilon=R$ and used inequality (23) of [EIR6]. Next,

$$
\begin{aligned}
N_{2 ; n}^{\prime}\left(\eta_{R} A_{k} \tau\right) & \leq N_{2 ; n+1}^{\prime}\left(\eta_{R} \tau\right)+N_{2 ; n}^{\prime}\left(\left(A_{k} \eta_{R}\right) \tau\right) \\
& \leq c^{\prime} R^{-n}\left(\int_{B^{\prime}(R)}\left|\nabla^{\prime} \tau\right|^{2}\right)^{1 / 2}=c^{\prime} R^{-n}\left(\sum_{i=1}^{d^{\prime}} \int_{B^{\prime}(R)}\left|A_{i} \varphi-\left\langle A_{i} \varphi\right\rangle_{R}\right|^{2}\right)^{1 / 2}
\end{aligned}
$$

by [ElR6, Lemma 3.1.II], where we used $H \tau=0$ weakly on $B^{\prime}(R)$ and $\langle\tau\rangle_{R}=0$. Combining these estimates one deduces that

$$
\sum_{k=1}^{d^{\prime}} \int_{B^{\prime}\left(\sigma^{-1} r\right)}\left|A_{k} \varphi-\left\langle A_{k} \varphi\right\rangle_{\sigma^{-1} r}\right|^{2} \leq c(r / R)^{D^{\prime}+2 \nu} \sum_{i=1}^{d^{\prime}} \int_{B^{\prime}(R)}\left|A_{i} \varphi-\left\langle A_{i} \varphi\right\rangle_{R}\right|^{2}
$$

for all $0<r \leq \sigma^{-1} R<R \leq R_{0}$ and the proof of the lemma is complete.

2.2.3. Operators with variable coefficients Finally we derive De Giorgi estimates involving derivatives for operators with $C^{v}$-coefficients. In the sequel we need various parameters to denote the smoothness of the coefficients of the subelliptic operator $H$. Hence we define the $C^{\nu}$-seminorm of the matrix $C$ of principal coefficients by

$$
\|C\|_{C^{\nu}}=\sup \left\{\left\|c_{i j}\right\|_{C^{\nu^{\prime}}}: i, j \in\left\{1, \ldots, d^{\prime}\right\}\right\},
$$

the $C^{v}$-norm of the first-order coefficients by

$$
\begin{aligned}
\|c\|_{C^{\nu^{\prime}}} & =\sup \left\{\left\|c_{i}\right\|_{C^{\nu^{\prime}}}: i \in\left\{1, \ldots, d^{\prime}\right\}\right\}, \\
\left\|c^{\prime}\right\| \|_{C^{\nu^{\prime}}} & =\sup \left\{\left\|c_{i}^{\prime}\right\|_{C^{\nu^{\prime}}}: i \in\left\{1, \ldots, d^{\prime}\right\}\right\}
\end{aligned}
$$

and the $L_{\infty}$-norm of the first-order coefficients by $\|c\|_{\infty}=\left(\sum_{i=1}^{d^{\prime}}\left\|c_{i}\right\|_{\infty}^{2}+\sum_{i=1}^{d^{\prime}}\left\|c_{i}^{\prime}\right\|_{\infty}^{2}\right)^{1 / 2}$. Note that the definition of $\|c\|_{\infty}$ involves both the $c_{i}$ and the $c_{i}^{\prime}$.

PROPOSITION 2.6. Let $v \in\langle 0,1\rangle$ and $H=-\sum_{i, j=1}^{d^{\prime}} A_{i} c_{i j} A_{j}$ a pure second-order subelliptic operator. Suppose either

(a) $H$ is strongly elliptic, or

(b) $G$ is stratified and $a_{1}, \ldots, a_{d^{\prime}}$ is a basis for $\mathfrak{g}_{1}$ in the stratification $\left(\mathfrak{g}_{m}\right)_{m \in\{1, \ldots, r\}}$ of $\mathfrak{g}$.

If $c_{i j} \in C^{\nu \prime}(G)$ then there exist $c_{D G}, c_{D G}^{\prime}>0$ such that for all $R \in\langle 0,1], g \in G$ and $\varphi \in H_{2: 1}^{\prime}\left(B^{\prime}(g ; R)\right)$ satisfying $H \varphi=0$ weakly on $B^{\prime}(g ; R)$ one has

$$
\begin{aligned}
& \sum_{k=1}^{d^{\prime}} \int_{B^{\prime}(g ; r)}\left|A_{k} \varphi-\left\langle A_{k} \varphi\right\rangle_{g, r}\right|^{2} \\
& \quad \leq c_{D G}(r / R)^{D^{\prime}+2 \bar{v}} \sum_{k=1}^{d^{\prime}} \int_{B^{\prime}(g ; R)}\left|A_{k} \varphi-\left\langle A_{k} \varphi\right\rangle_{g, R}\right|^{2}+c_{D G}^{\prime} R^{2 \nu} \int_{B^{\prime}(g ; R)}\left|\nabla^{\prime} \varphi\right|^{2}
\end{aligned}
$$


for all $0<r \leq R$, where $\tilde{v}=2^{-1}(1+v)$. Moreover, $c_{D G}$ and $c_{D G}^{\prime}$ depend on the coefficients of $H$ only through $\mu_{C},\|C\|_{\infty}$ and $\|C\|_{C^{v}}$.

PROOF. For $g \in G$ define the operator $H^{(g)}=-\sum_{i, j=1}^{d^{\prime}} A_{i} c_{i j}(g) A_{j}$ by fixing the coefficients. By Lemmas 2.4 and 2.5 it follows that there exist $c_{D G}>0$ and $c_{D G}^{\prime} \geq 0$ such that for all $R \in\langle 0,1]$, and $\eta \in H_{2 ; 1}^{\prime}\left(B^{\prime}(g ; R)\right)$ satisfying $H^{(g)} \eta=0$ weakly on $B^{\prime}(g ; R)$ one has

$$
\begin{aligned}
& \sum_{k=1}^{d^{\prime}} \int_{B^{\prime}(g ; r)}\left|A_{k} \eta-\left\langle A_{k} \eta\right\rangle_{g . r}\right|^{2} \\
& \quad \leq c_{D G}(r / R)^{D^{\prime}+2 \bar{v}} \sum_{k=1}^{d^{\prime}} \int_{B^{\prime}(g: R)}\left|A_{k} \eta-\left\langle A_{k} \eta\right\rangle_{g, R}\right|^{2}+c_{D G}^{\prime} R^{2} \int_{B^{\prime}(g ; R)}\left|\nabla^{\prime} \eta\right|^{2}
\end{aligned}
$$

for all $0<r \leq R$. The $c_{D G}$ and $c_{D G}^{\prime}$ depend only on $\mu_{C}$ and $\|C\|_{\infty}$ and are in particular independent of $g$.

Let $R \in\langle 0,1], g \in G, \varphi \in H_{2 ; 1}^{\prime}\left(B^{\prime}(g ; R)\right)$ and suppose $H \varphi=0$ weakly on $B^{\prime}(g ; R)$. Then let $\eta \in H_{2 ; 1}^{\prime}\left(B^{\prime}(g ; R)\right)$ be such that

$$
H^{(g)} \eta=0 \quad \text { weakly in } B^{\prime}(g ; R), \quad \chi=\varphi-\eta \in{\stackrel{\circ}{H_{2 ; 1}^{\prime}}}^{\prime}\left(B^{\prime}(g ; R)\right) .
$$

Arguing as in the proof of inequality (10) one deduces that

$$
\begin{aligned}
& \sum_{k=1}^{d^{\prime}} \int_{B^{\prime}(g ; r)}\left|A_{k} \varphi-\left\langle A_{k} \varphi\right\rangle_{g, r}\right|^{2} \\
& \leq 4 c_{D G}(r / R)^{D^{\prime}+2 \tilde{v}} \sum_{k=1}^{d^{\prime}} \int_{B^{\prime}(g ; R)}\left|A_{k} \varphi-\left\langle A_{k} \varphi\right\rangle_{g, R}\right|^{2} \\
& \quad+2 c_{D G}^{\prime} R^{2} \int_{B^{\prime}(g ; R)}\left|\nabla^{\prime} \eta\right|^{2}+\left(2+4 c_{D G}\right) \int_{B^{\prime}(g ; R)}\left|\nabla^{\prime} \chi\right|^{2} \\
& \leq 4 c_{D G}(r / R)^{D^{\prime}+2 \tilde{v}} \sum_{k=1}^{d^{\prime}} \int_{B^{\prime}(g ; R)}\left|A_{k} \varphi-\left\langle A_{k} \varphi\right\rangle_{g, R}\right|^{2} \\
& \quad+4 c_{D G}^{\prime} R^{2} \int_{B^{\prime}(g: R)}\left|\nabla^{\prime} \varphi\right|^{2}+\left(2+4 c_{D G}+4 c_{D G}^{\prime}\right) \int_{B^{\prime}(g ; R)}\left|\nabla^{\prime} \chi\right|^{2} .
\end{aligned}
$$

Next, since $\chi \in \stackrel{\circ}{H}_{2: 1}^{\prime}\left(B^{\prime}(g ; R)\right)$ and $H^{(g)} \chi=H^{(g)} \varphi$ one has as in [EIR6, Proposition 3.6]

$$
\begin{aligned}
\mu_{C} \int_{B^{\prime}(g ; R)}\left|\nabla^{\prime} \chi\right|^{2} & \leq \operatorname{Re}\left(\chi,\left(H^{(g)}-H_{P}\right) \varphi\right) \\
& \leq d^{\prime} \omega_{r}^{\prime}(C ; R)\left(\int_{B^{\prime}(g ; R)}\left|\nabla^{\prime} \chi\right|^{2}\right)^{1 / 2}\left(\int_{B^{\prime}(g ; R)}\left|\nabla^{\prime} \varphi\right|^{2}\right)^{1 / 2} \\
& \leq d^{\prime}\|C\|_{C^{v}} \cdot R^{v}\left(\int_{B^{\prime}(g ; R)}\left|\nabla^{\prime} \chi\right|^{2}\right)^{1 / 2}\left(\int_{B^{\prime}(g ; R)}\left|\nabla^{\prime} \varphi\right|^{2}\right)^{1 / 2} .
\end{aligned}
$$


Hence

$$
\int_{B^{\prime}(g ; R)}\left|\nabla^{\prime} \chi\right|^{2} \leq\left(d^{\prime}\|C\|_{C^{\nu}} \mu_{C}^{-1}\right)^{2} R^{2 \nu} \int_{B^{\prime}(g ; R)}\left|\nabla^{\prime} \varphi\right|^{2}
$$

and the proof of the proposition is complete.

\section{Operators with $C^{v}$-coefficients, $v<1$}

In this section we prove that Hölder continuity of the coefficients of $H$ ensures the corresponding semigroup kernel is differentiable with Hölder continuous derivatives. The proof is an extension of the arguments of [Aus] and [ElR6]. It relies on the De Giorgi estimates and uses Morrey and Campanato spaces. For a brief introduction to Morrey and Campanato spaces on Lie groups we refer to the appendix of [EIR6].

Proposition 3.1. Let $v \in\langle 0,1\rangle$ and let $H_{P}=-\sum_{i, j=1}^{d^{\prime}} A_{i} c_{i j} A_{j}$ be a pure secondorder subelliptic operator with $C^{\nu '}$-coefficients. Suppose either

(a) $H$ is strongly elliptic, or

(b) $G$ is stratified and $a_{1}, \ldots, a_{d^{\prime}}$ is a basis for $\mathfrak{g}_{1}$ in the stratification $\left(\mathfrak{g}_{m}\right)_{m \in\{1, \ldots, r\}}$ of $\mathfrak{g}$.

Let $\gamma \in\left[0, D^{\prime}\right\rangle, \eta \in\langle 0,2 v], \delta \in\langle 0,2]$ with $\eta \leq \gamma+\delta \leq D^{\prime}+\eta, \varphi \in L_{2 ; 1}^{\prime}$, $\tau \in M_{2, \gamma}$ and $\tau_{1}, \ldots, \tau_{d^{\prime}} \in \mathscr{M}_{2, \gamma+\delta}$. Suppose $A_{i} \varphi \in M_{2, \gamma+\delta-\eta}$ for all $i \in\left\{1, \ldots, d^{\prime}\right\}$ and $H_{P} \varphi=\tau+\sum_{i=1}^{d^{\prime}} A_{i} \tau_{i}$ weakly. Then $A_{i} \varphi \in \mathscr{M}_{2, \gamma+\delta}$ for all $i \in\left\{1, \ldots, d^{\prime}\right\}$ and there exists an $a>0$ such that

$$
\left\|\nabla^{\prime} \varphi\right\|_{\mathscr{M}_{2, \gamma+\delta}} \leq a\left(\varepsilon^{2-\delta}\|\tau\|_{M_{2, \gamma}}+\sum_{i=1}^{d^{\prime}}\left\|\tau_{i}\right\|_{\mathscr{M}_{2, \gamma+\delta}}+\left\|\nabla^{\prime} \varphi\right\|_{M_{2, \gamma+\delta-\eta}}+\varepsilon^{-(\gamma+\delta)}\|\varphi\|_{2 ; 1}^{\prime}\right)
$$

uniformly for all $\varepsilon>0$. The value of $a$ is independent of $\varphi, \tau$ and the $\tau_{i}$, and depends on $v$ and on $H_{P}$ through $\mu_{C},\|C\|_{\infty}$ and $\|C\|_{C^{\nu}}$.

PROOF. Let $c_{D G}$ and $c_{D G}^{\prime}$ be as in Proposition 2.6 and $R_{D}$ the radius and $c_{D}$ the constant in the Poincare inequalities for the Dirichlet Laplacian, Proposition 2.2 of [ElR6]. Let $g \in G$ and $0<r \leq R \leq R_{D}$. There exists an $\eta \in H_{2 ; 1}^{\prime}\left(B^{\prime}(g ; R)\right)$ such that

$$
H_{P} \eta=0 \quad \text { weakly in } B^{\prime}(g ; R), \quad \chi=\varphi-\eta \in \stackrel{\circ}{H_{2 ; 1}^{\prime}}\left(B^{\prime}(g ; R)\right) .
$$

Using Proposition 2.6 it follows as before that

$$
\begin{gathered}
\sum_{k=1}^{d^{\prime}} \int_{B^{\prime}(g: r)}\left|A_{k} \varphi-\left\langle A_{k} \varphi\right\rangle_{g, r}\right|^{2} \leq 2 c_{D G}(r / R)^{D^{\prime}+2 \tilde{v}} \sum_{k=1}^{d^{\prime}} \int_{B^{\prime}(g: R)}\left|A_{k} \varphi-\left\langle A_{k} \varphi\right\rangle_{g, R}\right|^{2} \\
+4 c_{D G}^{\prime} R^{2 v} \int_{B^{\prime}(g ; R)}\left|\nabla^{\prime} \varphi\right|^{2}+\left(2+4 c_{D G}+4 c_{D G}^{\prime}\right) \int_{B^{\prime}(g: R)}\left|\nabla^{\prime} \chi\right|^{2} .
\end{gathered}
$$


We estimate the two error terms in (11) separately. Since $A_{i} \varphi \in M_{2, \gamma+\delta-\eta}$ one has

$$
R^{2 v} \int_{B^{\prime}(g: R)}\left|\nabla^{\prime} \varphi\right|^{2} \leq R^{2 v}\left\|\nabla^{\prime} \varphi\right\|_{M_{2, \gamma+\delta-\eta}}^{2} R^{\gamma+\delta-\eta} \leq\left\|\nabla^{\prime} \varphi\right\|_{M_{2, y+\delta-\eta}}^{2} R^{\gamma+\delta}
$$

where we used that $\eta \leq 2 v$.

The integral of $\left|\nabla^{\prime} \chi\right|^{2}$ in (11) can be estimated as follows. By construction one has

$$
\left(\chi, H_{P} \chi\right)=\left(\chi, H_{P} \varphi\right)=(\chi, \tau)-\sum_{i=1}^{d^{\prime}}\left(A_{i} \chi, \tau_{i}\right)=(\chi, \tau)-\sum_{i=1}^{d^{\prime}}\left(A_{i} \chi, \tau_{i}-\left\langle\tau_{i}\right\rangle_{g, R}\right)
$$

since $\chi \in \stackrel{\circ}{H}_{2: 1}^{\prime}\left(B^{\prime}(g ; R)\right)$. Hence, by ellipticity and the Cauchy-Schwarz inequality,

$$
\begin{aligned}
\mu_{C} \int_{B^{\prime}(g: R)}\left|\nabla^{\prime} \chi\right|^{2} \leq & \left(\int_{B^{\prime}(g: R)}|\chi|^{2}\right)^{1 / 2}\left(\int_{B^{\prime}(g ; R)}|\tau|^{2}\right)^{1 / 2} \\
& +\sum_{i=1}^{d^{\prime}}\left(\int_{B^{\prime}(g: R)}\left|A_{i} \chi\right|^{2}\right)^{1 / 2}\left(\int_{B^{\prime}(g ; R)}\left|\tau_{i}-\left\langle\tau_{i}\right\rangle_{g \cdot R}\right|^{2}\right)^{1 / 2} \\
\leq & \|\tau\|_{M_{2 . \gamma}} R^{\gamma / 2}\left(c_{D} R^{2} \int_{B^{\prime}(g: R)}\left|\nabla^{\prime} \chi\right|^{2}\right)^{1 / 2} \\
& +\sum_{i=1}^{d^{\prime}}\left\|\tau_{i}\right\|_{\mathscr{M}_{2 . \gamma+\delta}} R^{(\gamma+\delta) / 2}\left(\int_{B^{\prime}(g: R)}\left|\nabla^{\prime} \chi\right|^{2}\right)^{1 / 2} .
\end{aligned}
$$

Therefore

$$
\int_{B^{\prime}(g: R)}\left|\nabla^{\prime} \chi\right|^{2} \leq \mu_{C}^{-2}\left(c_{D}^{1 / 2} R^{(2-\delta) / 2}\|\tau\|_{M_{2, \gamma}}+\sum_{i=1}^{d^{\prime}}\left\|\tau_{i}\right\|_{\mathscr{M}_{2 . \gamma+\delta}}\right)^{2} R^{\gamma+\delta} .
$$

Combining these estimates one deduces that

$$
\begin{aligned}
& \sum_{k=1}^{d^{\prime}} \int_{B^{\prime}(g: r)}\left|A_{k} \varphi-\left\langle A_{k} \varphi\right\rangle_{g, r}\right|^{2} \\
& \leq 4 c_{D G}(r / R)^{D^{\prime}+2 \tilde{\nu}} \sum_{k=1}^{d^{\prime}} \int_{B^{\prime}(g: R)}\left|A_{k} \varphi-\left\langle A_{k} \varphi\right\rangle_{g, R}\right|^{2} \\
& \quad+b\left(\left\|\nabla^{\prime} \varphi\right\|_{M_{2, \gamma+\delta-\eta}}+R^{(2-\delta) / 2}\|\tau\|_{M_{2, \gamma}}+\sum_{i=1}^{d^{\prime}}\left\|\tau_{i}\right\|_{\mathscr{M}_{2, \gamma+\delta}}\right)^{2} R^{\gamma+\delta} \\
& \leq 4 c_{D G}(r / R)^{D^{\prime}+2 \tilde{\nu}} \sum_{k=1}^{d^{\prime}} \int_{B^{\prime}(g: R)}\left|A_{k} \varphi-\left\langle A_{k} \varphi\right\rangle_{g, R}\right|^{2} \\
& \quad+b\left(\left\|\nabla^{\prime} \varphi\right\|_{M_{2, \gamma+\delta-\eta}}+\varepsilon^{2-\delta}\|\tau\|_{M_{2, \gamma}}+\sum_{i=1}^{d^{\prime}}\left\|\tau_{i}\right\|_{\mathscr{M}_{2, \gamma+\delta}}\right)^{2} R^{\gamma+\delta}
\end{aligned}
$$


uniformly for all $\varepsilon \in\langle 0,1]$ and $0<r \leq R \leq R_{D} \wedge \varepsilon^{2}$, where $b=4 c_{D G}^{\prime}+\mu_{C}^{-2}(1+$ $\left.c_{D}\right)\left(2+4 c_{D G}+4 c_{D G}^{\prime}\right)$. Since $\gamma+\delta<D^{\prime}+2 \tilde{v}$ one has, by [Gia2, Lemma III.2.1] (for a precise statement of the version we need, see [EIR6, Lemma 3.5]), there exists an $a>0$, depending only on $c_{D G}, \gamma+\delta$ and $v$, such that

$$
\begin{aligned}
\sum_{k=1}^{d^{\prime}} \int_{B^{\prime}(g ; r)}\left|A_{k} \varphi-\left\langle A_{k} \varphi\right\rangle_{g, r}\right|^{2} & \\
\leq a & \left((r / R)^{\gamma+\delta} \sum_{k=1}^{d^{\prime}} \int_{B^{\prime}(g ; R)}\left|A_{k} \varphi-\left\langle A_{k} \varphi\right\rangle_{g, R}\right|^{2}\right. \\
& \left.+b\left(\left\|\nabla^{\prime} \varphi\right\|_{M_{2, \gamma+\delta-\eta}}^{2}+\varepsilon^{2-\delta}\|\tau\|_{M_{2, \gamma}}+\sum_{i=1}^{d^{\prime}}\left\|\tau_{i}\right\|_{\mathscr{M}_{2, \gamma+\delta}}\right)^{2} r^{\gamma+\delta}\right)
\end{aligned}
$$

uniformly for all $g \in G$ and $0<r \leq R \leq R_{D} \wedge \varepsilon^{2}$. Choosing $R=R_{D} \varepsilon^{2}$ it follows that

$$
\begin{aligned}
\sum_{k=1}^{d^{\prime}} \int_{B^{\prime}(g: r)} & \left|A_{k} \varphi-\left\langle A_{k} \varphi\right\rangle_{g, r}\right|^{2} \\
\leq & a\left(R_{D}^{-(\gamma+2)} \varepsilon^{-2(\gamma+\delta)} \int_{G}\left|\nabla^{\prime} \varphi\right|^{2}\right. \\
& \left.\quad+b\left(\left\|\nabla^{\prime} \varphi\right\|_{M_{2, \gamma+\delta-\eta}}+\varepsilon^{2-\delta}\|\tau\|_{M_{2, \gamma}}+\sum_{i=1}^{d^{\prime}}\left\|\tau_{i}\right\|_{\mathscr{M}_{2, \gamma+\delta}}\right)^{2}\right) r^{\gamma+\delta}
\end{aligned}
$$

uniformly for $g \in G$ and $0<r \leq R_{D} \varepsilon^{2}$.

Finally, if $R_{D} \varepsilon^{2} \leq r \leq 1$ then

$$
\sum_{k=1}^{d^{\prime}} \int_{B^{\prime}(g ; r)}\left|A_{k} \varphi-\left\langle A_{k} \varphi\right\rangle_{g, r}\right|^{2} \leq \sum_{k=1}^{d^{\prime}} \int_{B^{\prime}(g ; r)}\left|A_{k} \varphi\right|^{2} \leq R_{D}^{-(\gamma+2)}\left(\varepsilon^{-(\gamma+\delta)}\|\varphi\|_{2 ; 1}^{\prime}\right)^{2}
$$

and combination of the last two inequalities completes the proof.

Next we consider estimates on the Davies perturbation of the semigroup $S$ generated by the subelliptic operator $H$ on $L_{2}$.

Let $\psi \in C_{c}^{\infty}(G)$ be a real-valued function and define the family of bounded multiplication operators $U_{\rho}, \rho \in \mathbb{R}$, by $U_{\rho} \varphi=e^{-\rho \psi} \varphi$. Then $S^{\rho}$ is the strongly continuous semigroup on $L_{2}$ given by $S_{t}^{\rho}=U_{\rho} S_{t} U_{\rho}^{-1}$ where we suppress the dependence on $\psi$ in the notation. The generator of $S_{t}^{\rho}$ is denoted by $H_{\rho}$. In [ElR6] we established, by an iterative argument, that $S^{\rho}$ is bounded from $L_{2}$ to $C_{\Delta}^{1-\varepsilon,}$ whenever the principal coefficients of the operator are uniformly continuous. Now we establish that if the coefficients are Hölder continuous then each $A_{i} S^{\rho}$ satisfies similar estimates. 
First, for each $\gamma \in\left[0, D^{\prime}+2\right)$, introduce the hypothesis $P(\gamma)$ by

$$
S_{t}^{\rho}\left(D^{\infty}\left(H_{\rho}\right)\right) \subset \mathscr{M}_{2, \gamma}, A_{i} S_{t}^{\rho}\left(D^{\infty}\left(H_{\rho}\right)\right) \subset \mathscr{M}_{2, \gamma} \text { for all } t>0, \rho \in \mathbb{R}
$$

and $i \in\left\{1, \ldots, d^{\prime}\right\}$. Moreover, there exist $b_{\gamma}, \omega_{\gamma}>0$, depending on $H$ through $\mu_{C},\|C\|_{\infty},\|C\|_{C^{\nu^{\prime}}},\|c\|_{\infty},\left.\left\|c^{\prime}\right\|\right|_{C^{v}}$, and $\left\|c_{0}\right\|_{\infty}$ such that

$$
\left\|S_{t}^{\rho} \varphi\right\|_{\mathscr{M}_{2 . \gamma}} \leq b_{\gamma} t^{-\gamma / 4} e^{\omega_{\gamma}\left(1+\rho^{2}\right) t}\|\varphi\|_{2}
$$

and

$$
\begin{gathered}
\left\|A_{i} S_{t}^{\rho} \varphi\right\|_{\mathscr{M}_{2, \gamma}} \leq b_{\gamma} t^{-\gamma / 4} t^{-1 / 2} e^{\omega_{\gamma}\left(1+\rho^{2}\right) t}\|\varphi\|_{2} \\
\text { for all } t>0, \rho \in \mathbb{R}, \varphi \in D^{\infty}\left(H_{\rho}\right) \text { and } i \in\left\{1, \ldots, d^{\prime}\right\}
\end{gathered}
$$

Since $L_{2} \cap M_{2, \gamma}=L_{2} \cap \mathscr{M}_{2, \gamma}$ for all $\gamma<D^{\prime}$, with equivalent norms, (see [EIR6, Lemma A.1.III]) we know that $P(\gamma)$ is valid in many cases. As an intermediate step in the proof of [EIR6, Proposition 4.3] we have already proved that $P(\gamma)$ is valid for all $\gamma \in\left[0, D^{\prime}\right\rangle$ if $D^{\prime} \geq 2$. (In fact we proved that the constants depend merely on the modulus of continuity $\omega_{r}^{\prime}(C ; \cdot)$ instead of $\|C\|_{C^{\prime}}$, and $\left\|c^{\prime}\right\| \|_{C^{\prime}}$.) In [ElR6] we used De Giorgi estimates which were valid only for $D^{\prime} \geq 2$. But the estimates of Section 2 are valid in any dimension. If $D^{\prime}=1$ then obviously $P(0)$ is valid. But then one can use Proposition 3.1 with $\eta=\delta$ instead of [EIR6, Proposition 4.2] and argue as in the proof of [ElR6, Lemma 4.4] to establish that $P(\gamma+\delta)$ is valid for all $\gamma \in\left[0, D^{\prime}\right\rangle$ and $\delta \in\langle 0,2 \nu]$ with $\gamma+\delta<D^{\prime}$, whenever $P(\gamma)$ is valid. Therefore $P(\gamma)$ is valid for all $\gamma \in\left[0, D^{\prime}\right)$ in case $D^{\prime}=1$. This argument also works in higher dimensions.

As in the proof of [EIR6, Proposition 4.3] it follows from (13) for $P(\gamma)$ with $\gamma \in\left\langle D^{\prime}-1, D^{\prime}\right\rangle$ that

$$
\left\|S_{t}^{\rho} \varphi\right\|_{\infty}^{\Delta} \leq a t^{-D^{\prime} / 4} e^{\omega\left(1+\rho^{2}\right) t}\|\varphi\|_{2}
$$

for all $\varphi \in D^{\infty}\left(H_{\rho}\right)$, where $a$ and $\omega$ depend only on the allowed parameters.

The main step in the proof of the new proposition is the observation that Hölder continuity of the coefficients ensures $P(\gamma)$ is valid for all $\gamma \in\left[0, D^{\prime}+2 v\right]$.

For $\psi \in C_{c}^{\infty}(G)$ set

$$
n_{2}(\psi)=\sup _{i \in\left\{1, \ldots, d^{\prime}\right\}}\left\|A_{i} \psi\right\|_{\infty} \vee \sup _{i, j \in\left\{1, \ldots, d^{\prime}\right\}}\left\|A_{i} A_{j} \psi\right\|_{\infty}
$$

PROPOSITION 3.2. Let $H$ be a subelliptic operator in divergence form (1). Suppose either

(a) $H$ is strongly elliptic, or

(b) $G$ is stratified and $a_{1}, \ldots, a_{d^{\prime}}$ is a basis for $\mathfrak{g}_{1}$ in the stratification $\left(\mathfrak{g}_{m}\right)_{m \in\{1, \ldots, r\}}$ of $\mathrm{g}$. 
If $c_{i j}, c_{i}^{\prime} \in C^{\nu \prime}$ and $c_{i}, c_{0} \in L_{\infty}$ for some $v \in\langle 0,1\rangle$ then $A_{i} S_{t}^{\rho} L_{2} \subset C_{\Delta}^{\nu '}(G) \cap$ $L_{\infty}^{\Delta}(G)$ for all $t>0$ and $\rho \in \mathbb{R}$ and there exist $a, \omega>0$ such that

$$
\left\|A_{i} S_{t}^{\rho} \varphi\right\|_{\infty}^{\Delta} \leq a t^{-\left(D^{\prime}+2\right) / 4} e^{\omega\left(1+\rho^{2}\right) t}\|\varphi\|_{2}
$$

and

$$
\left\|A_{i} S_{t}^{\rho} \varphi\right\|_{C_{\Delta}^{\nu}} \leq a t^{-\left(D^{\prime}+2\right) / 4} t^{-\nu / 2} e^{\omega\left(1+\rho^{2}\right) t}\|\varphi\|_{2}
$$

uniformly for $\varphi \in L_{2}(G), t>0, \rho \in \mathbb{R}$ and $i \in\left\{1, \ldots, d^{\prime}\right\}$. The constants $a$ and $\omega$ depend on $v$ and on $H$ through $\mu_{C},\|C\|_{\infty},\|C\|_{C^{\prime}},\|c\|_{\infty},\left.\left\|c^{\prime}\right\|\right|_{C^{\nu}}$ and $\left\|c_{0}\right\|_{\infty}$. They depend on the perturbation functions $\psi \in C_{c}^{\infty}(G)$ only through $n_{2}(\psi)$.

PROOF. Set $\delta=\min \left(2,2^{-1}+2 v\right)$ and let $\gamma \in\left\langle\left(D^{\prime}-\delta\right) \vee 0, D^{\prime}+2 \nu-\delta\right\rangle$. Note that this interval is not empty. We show that $P(\gamma+\delta)$ is valid. Fix $\varphi \in D^{\infty}\left(H_{\rho}\right)$. Then (12) for $P(\gamma+\delta)$ follows from (13) and the Poincaré inequality, [EIR6, Lemma A.2], in a similar manner to the proof of [EIR6, Lemma 4.4].

Next we use the decomposition

$$
H_{P} S_{t}^{\rho} \varphi=S_{t}^{\rho} H_{\rho} \varphi-c_{0}^{(\rho)} S_{t}^{\rho} \varphi-\sum_{i=1}^{d^{\prime}} c_{i}^{(\rho)} A_{i} S_{t}^{\rho} \varphi-\sum_{i=1}^{d^{\prime}} A_{i}{c_{i}^{\prime}}^{(\rho)} S_{t}^{\rho} \varphi
$$

with perturbed lower-order coefficients as in [EIR6, Lemma 4.4]. This time we apply Proposition 3.1 to $S_{t}^{\rho} \varphi$ with $\tau$ again the first three terms on the right, $\tau_{i}=c_{i}^{(\rho)} S_{t}^{\rho} \varphi$ and $\eta=2 v$. The proof of (13) for $P(\gamma+\delta)$ is almost the same as the comparable proof in [EIR6, Lemma 4.4], so we indicate the differences.

First we have the term $\left\|\nabla^{\prime} S_{t}^{o} \varphi\right\|_{M_{2, \gamma+2-\eta}}$ which is not present in [ElR6]. Since $\gamma<D^{\prime}+2 v-\delta$ one has $\gamma+\delta-2 v<D^{\prime}$ and hence by (13) for $P(\gamma+\delta-2 v)$ one has

$$
\begin{aligned}
\left\|\nabla^{\prime} S_{t}^{\rho} \varphi\right\|_{M_{2, \gamma+\delta-\eta}} & \leq b_{\gamma+\delta-2 v} t^{-(\gamma+\delta-2 v) / 4} t^{-1 / 2} e^{\omega_{\gamma+\delta-2 v}\left(1+\rho^{2}\right) t}\|\varphi\|_{2} \\
& \leq a^{\prime} t^{-(\gamma+\delta) / 4} t^{-1 / 2} e^{\omega^{\prime}\left(1+\rho^{2}\right) t}\|\varphi\|_{2} .
\end{aligned}
$$

Secondly, we have to estimate the $\left\|\tau_{i}\right\|_{\mathscr{M}_{2, \gamma+\delta}}$. Since $\gamma+\delta>D^{\prime}$ it follows from [ElR6, Lemma A.1.V] that there exists a constant $a>0$, depending only on $\gamma+\delta$, such that

$$
\left\|{c_{i}^{\prime}}^{(\rho)} S_{t}^{\rho} \varphi\right\|_{\mathscr{M}_{2, \gamma+\delta}} \leq a\left\|c_{i}^{\prime(\rho)} S_{t}^{\rho} \varphi\right\|_{C_{\Delta}^{\mu}},
$$

where we have set $\mu=\left(\gamma+\delta-D^{\prime}\right) / 2$. Moreover,

$$
\|\| c_{i}^{(\rho)} S_{t}^{\rho} \varphi\left\|_{C_{\Delta}^{\mu}} \leq\right\|\left\|c_{i}^{\prime(\rho)}\right\|\left\|_{C^{\mu}},\right\| S_{t}^{\rho} \varphi\left\|_{\infty}^{\Delta}+\right\| c_{i}^{\prime(\rho)}\left\|_{\infty}\right\|\left\|S_{t}^{\rho} \varphi\right\|_{C_{\Delta}^{\mu}}
$$


and

$$
\begin{aligned}
\left\|c_{i}^{\prime(\rho)}\right\|_{C^{\mu}} & \leq\left(1+\rho^{2}\right)^{1 / 2}\left(\left\|c_{i}^{\prime}\right\|_{C^{\mu}}+\sum_{j=1}^{d^{\prime}}\left(\left\|\psi_{j}\right\|_{\infty}\left\|c_{i j}\right\|_{C^{\mu}}+\left\|\psi_{j}\right\|\left\|_{C^{\mu}}\right\| c_{i j} \|_{\infty}\right)\right) \\
& \leq a^{\prime} t^{-1 / 2} e^{\omega\left(1+\rho^{2}\right) t}\left(\left\|c_{i}^{\prime}\right\|_{C^{\nu}}+\sum_{j=1}^{d^{\prime}}\left\|\psi_{j}\right\|_{\infty: 1}^{\prime}\left(\left\|c_{i j}\right\|\left\|_{C^{\nu}}+\right\| c_{i j} \|_{\infty}\right)\right)
\end{aligned}
$$

for suitable $a^{\prime}$ and $\omega$, by Lemma 2.1 and (6). Together with the bounds (14) one deduces that

$$
\left\|{c_{i}^{\prime}}^{(\rho)}\right\|\left\|_{C^{\mu}}\right\| S_{t}^{\rho} \varphi\left\|_{\infty}^{\Delta} \leq a t^{-D^{\prime} / 4} t^{-1 / 2} e^{\omega\left(1+\rho^{2}\right) t}\right\| \varphi\left\|_{2} \leq a^{\prime} t^{-(\gamma+\delta) / 4} t^{-1 / 2} e^{\omega^{\prime}\left(1+\rho^{2}\right) t}\right\| \varphi \|_{2}
$$

for suitable $a, a^{\prime}, \omega$ and $\omega^{\prime}$, depending only on $\nu, \mu_{C},\|C\|_{\infty},\|C\|_{C^{\nu}},\|c\|_{\infty},\left\|c^{\prime}\right\|_{C^{\nu}}$, $\left\|c_{0}\right\|_{\infty}\left\|A_{i} \psi\right\|_{\infty}$ and $\left\|A_{i} A_{j} \psi\right\|_{\infty}$.

Alternatively, by [ElR6, Lemma A.1.V], and (12) for $P(\gamma)$

$$
\left\|S_{t}^{\rho} \varphi\right\|_{C_{\Delta}^{\mu}} \leq a\left(\left\|S_{t}^{\rho} \varphi\right\|_{\mathscr{M}_{2, \gamma+\delta}}+\left\|S_{t}^{\rho}\right\|_{2}\right) \leq a^{\prime} t^{-(\gamma+\delta) / 4} e^{\omega\left(1+\rho^{2}\right) t}\|\varphi\|_{2} .
$$

Combining these estimates one deduces that

$$
\left\|{c_{i}^{\prime}}^{(\rho)} S_{t}^{\rho} \varphi\right\|_{\mathscr{M}_{2, \gamma+\delta}} \leq a t^{-(\gamma+\delta) / 4} t^{-1 / 2} e^{\omega\left(1+\rho^{2}\right) t}\|\varphi\|_{2}
$$

for suitable $a$ and $\omega$, as required. Thus (13) for $P(\gamma+\delta)$ follows for all $\gamma$ in the interval $\left\langle\left(D^{\prime}-\delta\right) \vee 0, D^{\prime}+2 v-\delta\right\rangle$.

Now let $\gamma \in\left\langle D^{\prime} \vee \delta, D^{\prime}+2 \nu\right\rangle$. Then by Lemma A.3 of [ElR6] and (13) for $P(\gamma)$ one has

$$
\left\|A_{i} S_{t}^{\rho} \varphi\right\|_{\infty}^{\Delta} \leq \varepsilon^{\left(\gamma-D^{\prime}\right) / 2}\left\|A_{i} S_{t}^{\rho} \varphi\right\|_{\mathscr{M}_{2 . \gamma}}+c \varepsilon^{-D^{\prime} / 2}\left\|A_{i} S_{t}^{\rho} \varphi\right\|_{2} \leq a t^{-D^{\prime} / 4} t^{-1 / 2} e^{\omega\left(1+\rho^{2}\right) t}\|\varphi\|_{2}
$$

for suitable $a$ and $\omega$, by setting $\varepsilon=t^{1 / 2}$. But then

$$
\left\|A_{i} S_{t}^{\rho} \varphi\right\|_{M_{2, D^{\prime}}} \leq a^{\prime} t^{-D^{\prime} / 4} t^{-1 / 2} e^{\omega\left(1+\rho^{2}\right) t}\|\varphi\|_{2}
$$

by an application of [EIR6, Lemma A.I.IV].

Finally we prove $P\left(D^{\prime}+2 v\right)$. We argue as above, with $\gamma=D^{\prime}+2 v-\delta$, but in the first step we now use

$$
\left\|\nabla^{\prime} S_{t}^{\rho} \varphi\right\|_{M_{2, v+\delta-2 v}}=\left\|\nabla^{\prime} S_{t}^{\rho} \varphi\right\|_{M_{2, D^{\prime}}} \leq a t^{-D^{\prime} / 4} t^{-1 / 2} e^{\omega\left(1+\rho^{2}\right) t}\|\varphi\|_{2} .
$$

This was the only place where we previously used $\gamma \neq D^{\prime}-\delta+2 v$. The details are left to the reader. 
It remains to translate the semigroup bounds into kernel bounds. Since we have to do this repeatedly in the sequel as we assume more smoothness of the coefficients, we immediately treat the general case of divergence form operators.

For $N_{2}, N_{1}, N_{1}^{\prime}, N_{0} \in \mathbb{N}_{0}, v_{2}, v_{1}, v_{1}^{\prime}, v_{0} \in[0,1\rangle, \mu>0$ and $M>0$ such that $N_{2}+v_{2}>0$,

$$
N_{2}+v_{2} \geq N_{1}+v_{1} \geq N_{0}+v_{0}, \quad N_{2}+v_{2} \geq N_{1}^{\prime}+v_{1}^{\prime} \geq N_{0}+v_{0}
$$

let $\mathscr{E}^{\text {div }}\left(N_{2}+v_{2}, N_{1}+v_{1}, N_{1}^{\prime}+v_{1}^{\prime}, N_{0}+v_{0}, \mu, M\right)$ be the set of all second-order subelliptic operators of the form

$$
H=-\sum_{i, j=1}^{d^{\prime}} A_{i} c_{i j} A_{j}+\sum_{i=1}^{d^{\prime}}\left(c_{i} A_{i}+A_{i} c_{i}^{\prime}\right)+c_{0} I
$$

such that $c_{i j} \in L_{\infty ; N_{2}}^{\prime},\left\|c_{i j}\right\|_{\infty ; N_{2}}^{\prime} \leq M$, the ellipticity constant $\mu_{C} \geq \mu$ and if $\nu_{2}>0$ then $\left\|A^{\alpha} c_{i j}\right\|_{C^{\nu^{2}}}, \leq M$ for all $\alpha \in J_{N_{2}}\left(d^{\prime}\right)$ and similar conditions on the $c_{i}, c_{i}^{\prime}$ and $c_{0}$. Since $N_{2}+\nu_{2}>0$ it is clear that $H$ and $H^{*}$ generate semigroups on all the $L_{p}$-spaces.

Because of the inequalities (17) it follows that $H_{\rho} \in \bigcup_{M>0} \mathscr{E}^{\text {div }}\left(N_{2}+v_{2}, N_{1}+\right.$ $\left.v_{1}, N_{1}^{\prime}+v_{1}^{\prime}, N_{0}+v_{0}, \mu, M\right)$ for all $H \in \bigcup_{M>0} \mathscr{E}^{\text {div }}\left(N_{2}+v_{2}, N_{1}+v_{1}, N_{1}^{\prime}+v_{1}^{\prime}, N_{0}+\right.$ $\left.v_{0}, \mu, M\right), \rho \in \mathbb{R}, \mu>0$ and $\psi \in C_{c}^{\infty}(G)$. Moreover, for all $M>0$ there exists an $M^{\prime}>0$ such that $H^{\Delta} \in \mathscr{E}^{\text {div }}\left(N_{2}+v_{2}, N_{1}+v_{1}, N_{1}^{\prime}+v_{1}^{\prime}, N_{0}+v_{0}, \mu, M^{\prime}\right)$ for all $H \in \mathscr{E}^{\text {div }}\left(N_{2}+v_{2}, N_{1}+v_{1}, N_{1}^{\prime}+v_{1}^{\prime}, N_{0}+v_{0}, \mu, M\right)$, where the operator $H^{\Delta}$, defined at the end of [EIR6, Subsection 2.4], is formally given by $H^{\Delta}=\Delta^{-1 / 2} H \Delta^{1 / 2}$. The generator $H^{\Delta}$ is obtained by replacing each $A_{i}$ by $A_{i}+2^{-1} b_{i} I$ in $H$, where $b_{i}=\left(A_{i} \Delta\right)(e)$.

Obviously $H^{*} \in \mathscr{E}^{\text {div }}\left(N_{2}+v_{2}, N_{1}^{\prime}+v_{1}^{\prime}, N_{1}+v_{1}, N_{0}+v_{0}, \mu, M\right)$ if, and only if, $H \in \mathscr{E}^{\mathrm{div}}\left(N_{2}+v_{2}, N_{1}+v_{1}, N_{1}^{\prime}+\nu_{1}^{\prime}, N_{0}+v_{0}, \mu, M\right)$.

We reformulate Proposition 3.2 and weaken an intermediate result of [EIR6]:

PROPOSITION 3.3. Adopt the hypotheses of Proposition 3.2.

Let $v>0$. Then for all $M, \mu>0$ there exist a, $\omega>0$ such that $A_{i} S_{t}^{\rho} C_{c}^{\infty}(G) \subset$ $C^{\nu \prime}(G) \cap L_{\infty}(G)$ for all $t>0$ and $\rho \in \mathbb{R}$,

$$
\left\|A_{i} S_{1}^{\rho} \varphi\right\|_{\infty} \leq a t^{-\left(D^{\prime}+2\right) / 4} e^{\omega\left(1+\rho^{2}\right) t}\|\varphi\|_{\hat{2}},
$$

and

$$
\left.\left\|A_{i} S_{t}^{\rho} \varphi\right\|\right|_{C^{\prime \prime}} \leq a t^{-\left(D^{\prime}+2\right) / 4} t^{-v / 2} e^{\omega\left(1+\rho^{2}\right) t}\|\varphi\|_{\hat{2}}
$$

uniformly for all $H \in \mathscr{E}^{\text {div }}(\nu, 0, \nu, 0, \mu, M), \varphi \in C_{c}^{\infty}(G), t>0, i \in\left\{1, \ldots, d^{\prime}\right\}$, $\rho \in \mathbb{R}$ and $\psi \in C_{c}^{\infty}(G)$ with $n_{2}(\psi) \leq 1$.

PROOF. This follows as in the proof of [EIR6, Proposition 4.4]. 
PROPOSITION 3.4. Adopt the hypotheses of Proposition 3.2. Let $v>0$. Then for all $M, \mu>0$ there exist $a, \omega>0$ such that $S_{t}^{\rho} C_{c}^{\infty}(G) \subset C^{\nu}(G) \cap L_{\infty}(G)$ for all $t>0$ and $\rho \in \mathbb{R}$,

$$
\left\|S_{t}^{\rho} \varphi\right\|_{\infty} \leq a t^{-D^{\prime} / 4} e^{\omega\left(1+\rho^{2}\right) t}\|\varphi\|_{\hat{2}},
$$

and

$$
\|\| S_{t}^{\rho} \varphi\|\|_{C^{\nu}} \leq a t^{-D^{\prime} / 4} t^{-v / 2} e^{\omega\left(1+\rho^{2}\right) t}\|\varphi\|_{2}
$$

uniformly for all $H \in \mathscr{E}^{\text {div }}(\nu, 0,0,0, \mu, M), \varphi \in C_{c}^{\infty}(G), t>0, \rho \in \mathbb{R}$ and $\psi \in C_{c}^{\infty}(G)$ with $n_{2}(\psi) \leq 1$.

PROOF. This follows from the inequalities (30) and (34) in [ElR6]. In fact, one only needs a uniform bound on the modulus of right continuity of the second-order coefficients.

The next proposition establishes that one has kernel bounds starting from bounds on derivatives of $S_{t}^{\rho}$ and $S_{t}^{* \rho}=U_{\rho} e^{-t H^{*}} U_{\rho}^{-1}$, provided these bounds are uniform for all $H$ in a set $\mathscr{E}^{\text {div }}\left(N_{2}+v_{2}, N_{1}+v_{1}, N_{1}^{\prime}+v_{1}^{\prime}, N_{0}+v_{0}, \mu, M\right)$ for each $\mu$ and $M$. The seemingly surprising fact is that for this proposition there is no relation needed between the number of derivatives posed on $S_{t}^{\rho}$ and $S_{t}^{* \rho}$ and the $N_{2}, v_{2}$, etcetera.

Proposition 3.5. Fix $N, N^{*} \in \mathbb{N}_{0}$ and $\nu, v^{*} \in\langle 0,1\rangle$. Next, let $N_{2}, N_{1}, N_{1}^{\prime}, N_{0} \in$ $\mathbb{N}_{0}, v_{2}, v_{1}, v_{1}^{\prime}, v_{0} \in[0,1\rangle, \mu>0$ and $N^{\prime} \in \mathbb{N}$. Assume $N_{2}+v_{2}>0$ and the inequalities (17). Suppose for all $M>0$ there exist $a>0$ and $\omega \geq 0$ such that $S_{t}^{\rho} C_{c}^{\infty}(G) \subset L_{2: N}^{\prime}, A^{\alpha} S_{t}^{\rho} C_{c}^{\infty}(G) \subset C^{\nu}(G) \cap L_{\infty}(G)$ for all $\alpha \in J_{N}\left(d^{\prime}\right)$, where $A$ is the $L_{2}$-derivative, $S_{t}^{* \rho} C_{c}^{\infty}(G) \subset L_{2 ; N^{*}}^{\prime}, A^{\beta} S_{t}^{* \rho} C_{c}^{\infty}(G) \subset C^{\nu^{*} \prime}(G) \cap L_{\infty}(G)$ for all $\beta \in J_{N^{*}}\left(d^{\prime}\right)$,

$$
\begin{aligned}
\left\|A^{\alpha} S_{t}^{\rho} \varphi\right\|_{\infty} & \leq a t^{-D^{\prime} / 4} t^{-|\alpha| / 2} e^{\omega\left(1+\rho^{2}\right) t}\|\varphi\|_{\hat{2}}, \\
\left\|A^{\alpha} S_{t}^{\rho} \varphi\right\|_{C^{\prime}} & \leq a t^{-D^{\prime} / 4} t^{-(|\alpha|+\nu) / 2} e^{\omega\left(1+\rho^{2}\right) t}\|\varphi\|_{\hat{2}}, \\
\left\|A^{\beta} S_{t}^{* \rho} \varphi\right\|_{\infty} & \leq a t^{-D^{\prime} / 4} t^{-|\beta| / 2} e^{\omega\left(1+\rho^{2}\right) t}\|\varphi\|_{\hat{2}}, \\
\left\|A^{\beta} S_{t}^{* \rho} \varphi\right\|_{C^{*},} & \leq a t^{-D^{\prime} / 4} t^{-\left(|\beta|+\nu^{*}\right) / 2} e^{\omega\left(1+\rho^{2}\right) t}\|\varphi\|_{\hat{2}},
\end{aligned}
$$

uniformly for all $H \in \mathscr{E}^{\text {div }}\left(N_{2}+v_{2}, N_{1}+v_{1}, N_{1}^{\prime}+v_{1}^{\prime}, N_{0}+v_{0}, \mu, M\right), \varphi \in C_{c}^{\infty}(G)$, $\alpha \in J_{N}\left(d^{\prime}\right), \beta \in J_{N^{*}}\left(d^{\prime}\right), \rho \in \mathbb{R}$ and $\psi \in C_{c}^{\infty}(G)$ with $\left\|A^{\gamma} \psi\right\|_{\infty} \leq 1$ for all $\gamma \in J\left(d^{\prime}\right)$ with $1 \leq|\gamma| \leq N^{\prime}$.

Then for all $\kappa>0$ and $M>0$ there exist $a, b>0$ and $\omega \geq 0$, such that for each $H \in \mathscr{E}^{\text {div }}\left(N_{2}+v_{2}, N_{1}+v_{1}, N_{1}^{\prime}+v_{1}^{\prime}, N_{0}+v_{0}, \mu, M\right)$ the kernel $K$ of the semigroup $S$ generated by $H$ is $N$-times differentiable in the first variable, the derivatives with respect to the first are $N^{*}$-times differentiable with respect to the second, the derivatives are continuous,

$$
\left|\left(A^{\alpha} B^{\beta} K_{t}\right)(g ; h)\right| \leq a t^{-D^{\prime} / 2} t^{-(|\alpha|+|\beta|) / 2} e^{\omega t} e^{-b\left(\mid g h^{-1} l^{\prime}\right)^{2} t^{-1}}
$$


and

$$
\left|\left(A^{\alpha} B^{\beta} K_{t}\right)\left(k^{-1} g ; l^{-1} h\right)-\left(A^{\alpha} B^{\beta} K_{t}\right)(g ; h)\right|
$$

$$
\leq a t^{-D^{\prime} / 2} t^{-(|\alpha|+|\beta|) / 2} e^{\omega t}\left(\left(\frac{|k|^{\prime}}{t^{1 / 2}+\left|g h^{-1}\right|^{\prime}}\right)^{\nu}+\left(\frac{|l|^{\prime}}{t^{1 / 2}+\left|g h^{-1}\right|^{\prime}}\right) e^{v^{*}}\right) e^{-b\left(\left.g h^{-1}\right|^{\prime}\right)^{2} t^{-1}}
$$

uniformly for all $\alpha \in J_{N}\left(d^{\prime}\right), \beta \in J_{N^{*}}\left(d^{\prime}\right), t>0, g, h \in G$ and $k, l \in G$ such that $|k|^{\prime}+|l|^{\prime} \leq \kappa t^{1 / 2}+2^{-1}\left|g h^{-1}\right|^{\prime}$.

PROOF. The proof of this proposition is an elaboration of the proof of [ElR6, Proposition 4.5], so again we indicate the differences. Let $A_{i}^{\rho}=U_{\rho} A_{i} U_{\rho}^{-1}$. It follows from [Rob, p. 191], that for all $\alpha \in J_{N}\left(d^{\prime}\right)$ there exist $c_{\alpha \beta}(\rho ; \psi) \in \mathbb{R}$ such that $\left(A^{\rho}\right)^{\alpha}=\sum_{|\beta| \leq|\alpha|} c_{\alpha \beta}(\rho ; \psi) A^{\beta}$ with $\rho \mapsto c_{\alpha \beta}(\rho ; \psi)$ a polynomial of order $|\alpha|-|\beta|$, in $\rho$, which depends on $\psi$ only through the derivatives $A^{\gamma} \psi$ with $|\gamma| \in\{1, \ldots,|\alpha|-|\beta|\}$. So

$$
\begin{aligned}
\left\|\left(A^{\rho}\right)^{\alpha} S_{t}^{\rho} \varphi\right\|_{\infty} & \leq \sum_{|\beta| \leq|\alpha|}\left|c_{\alpha \beta}(\rho ; \psi)\right|\left\|A^{\beta} S_{\imath}^{\rho} \varphi\right\|_{\infty} \\
& \leq \sum_{|\beta| \leq|\alpha|} a^{\prime}\left(1+\rho^{2}\right)^{(|\alpha|-|\beta|) / 2} a t^{-D^{\prime} / 4} t^{-|\beta| / 2} e^{\omega\left(1+\rho^{2}\right) t}\|\varphi\|_{\hat{2}} \\
& \leq a^{\prime \prime} t^{-D^{\prime} / 4} \sum_{|\beta| \leq|\alpha|} t^{-(|\alpha|-|\beta|) / 2} t^{-|\beta| / 2} e^{\omega^{\prime}\left(1+\rho^{2}\right) t}\|\varphi\|_{\hat{2}} \\
& =a^{\prime \prime} t^{-D^{\prime} / 4} t^{-|\alpha| / 2} e^{\omega^{\prime}\left(1+\rho^{2}\right) t}\|\varphi\|_{\hat{2}} .
\end{aligned}
$$

Here $a^{\prime \prime}$ depends on $\psi$ only through the $\left\|A^{\gamma} \psi\right\|_{\infty}$ with $|\gamma| \in\left\{1, \ldots, N \vee N^{\prime}\right\}$. Similarly,

$$
\begin{aligned}
&\left\|(I-L(k))\left(A^{\rho}\right)^{\alpha} S_{t}^{\rho} \varphi\right\|_{\infty} \leq \sum_{|\beta| \leq|\alpha|}\left\|(I-L(k)) c_{\alpha \beta}(\rho ; \psi) A^{\beta} S_{t}^{\rho} \varphi\right\|_{\infty} \\
& \leq \sum_{|\beta| \leq|\alpha|}\left(\left\|(I-L(k)) c_{\alpha \beta}(\rho ; \psi)\right\|_{\infty}\left\|A^{\beta} S_{t}^{\rho} \varphi\right\|_{\infty}\right. \\
&\left.\quad+\left\|L(k) c_{\alpha \beta}(\rho ; \psi)\right\|_{\infty}\left\|(I-L(k)) A^{\beta} S_{t}^{\rho} \varphi\right\|_{\infty}\right)
\end{aligned}
$$

by (5). Now $\left\|(I-L(k)) c_{\alpha \beta}(\rho ; \psi)\right\|_{\infty} \leq a^{\prime}|k|^{\prime}\left(1+\rho^{2}\right)^{(|\alpha|-|\beta|) / 2}$, where $a^{\prime}$ depends on $\psi$ only through the $\left\|A^{\gamma} \psi\right\|_{\infty}$ with $|\gamma| \in\{2, \ldots,|\alpha|-|\beta|+1\}$ by (6). Therefore

$$
\begin{aligned}
\left\|(I-L(k)) c_{\alpha \beta}(\rho ; \psi)\right\|_{\infty}\left\|A^{\beta} S_{t}^{\rho} \varphi\right\|_{\infty} & \leq a^{\prime}|k|^{\prime}\left(1+\rho^{2}\right)^{\{|\alpha|-|\beta|) / 2} a t^{-D^{\prime} / 4} t^{-|\beta| / 2} e^{\omega\left(1+\rho^{2}\right) t}\|\varphi\|_{\hat{2}} \\
& \leq a^{\prime \prime}|k|^{\prime} t^{-D^{\prime} / 4} t^{-\{\alpha \mid / 2} e^{\omega^{\prime}\left(1+\rho^{2}\right) r}\|\varphi\|_{\hat{2}} \\
& \leq a^{\prime \prime \prime}\left(|k|^{\prime}\right)^{v} t^{-D^{\prime} / 4} t^{-(|\alpha|+\nu) / 2} e^{\omega^{\prime \prime}\left(1+\rho^{2}\right) t}\|\varphi\|_{\hat{2}}
\end{aligned}
$$


for all $k \in B^{\prime}(1)$ and $t>0$. The second term in (25) can be estimated similarly and hence for all $M>0$ there exist $a>0$ and $\omega \geq 0$ such that

$$
\left\|(I-L(k)) A^{\rho \alpha} S_{t}^{\rho} \varphi\right\|_{\infty} \leq a\left(|k|^{\prime}\right)^{\nu} t^{-D^{\prime} / 4} t^{-(|\alpha|+\nu) / 2} e^{\omega\left(1+\rho^{2}\right) t}\|\varphi\|_{2}
$$

uniformly for all $H \in \mathscr{E}^{\text {div }}\left(N_{2}+v_{2}, N_{1}+v_{1}, N_{1}^{\prime}+v_{1}^{\prime}, N_{0}+v_{0}^{\prime}, \mu, M\right), \varphi \in C_{c}^{\infty}(G)$, $\alpha \in J_{N}\left(d^{\prime}\right), k \in B^{\prime}(1), \rho \in \mathbb{R}$ and $\psi \in C_{c}^{\infty}(G)$ with $\left\|A^{\beta} \psi\right\|_{\infty} \leq 1$ for all $\beta \in J\left(d^{\prime}\right)$ with $1 \leq|\beta| \leq N \vee N^{\prime}+1$. But then it follows from (24) that these estimates are valid for all $k \in G$ by increasing $a, \omega$ and $N^{\prime}$.

Now let $S^{\rho \hat{*}}$ denote the $L_{\hat{2}}$-adjoint of $S^{\rho}$. Then $S^{\rho \hat{*}}$ is the semigroup generated by the subelliptic operator $\left(H^{*}\right)_{-\rho}$, where $H^{\hat{*}}$ is formally given by $H^{\hat{*}}=\left(\Delta^{-1} H \Delta\right)^{*}$. Precisely, $H^{\hat{*}}=H^{\Delta \Delta *}$. Hence, arguing as above, given $M>0$ there exist $a>0$ and $\omega \geq 0$ such that

$$
\left|\left\langle\varphi_{1}, S_{t}^{\rho} \widehat{A}^{\rho \beta} \varphi_{2}\right\rangle\right|=\left|\left\langle A^{\rho \beta} S^{\rho \hat{*}} \varphi_{1}, \varphi_{2}\right\rangle\right| \leq a t^{-D^{\prime} / 4} t^{-|\beta| / 2} e^{\omega\left(1+\rho^{2}\right) t}\left\|\varphi_{1}\right\|_{\hat{2}}\left\|\varphi_{2}\right\|_{\hat{i}}
$$

for all $\varphi_{1}, \varphi_{2} \in C_{c}^{\infty}(G)$, where $\widehat{A_{i}}=A_{i}-b_{i} l$. Therefore

$$
\left\|S_{t}^{\rho}\left(\widehat{A}^{\rho}\right)^{\beta} \varphi\right\|_{\hat{2}} \leq a t^{-D^{\prime} / 4} t^{-|\beta| / 2} e^{\omega\left(1+\rho^{2}\right) t}\|\varphi\|_{\hat{1}}
$$

and similarly,

$$
\left\|S_{t}^{\rho}\left(\widehat{A}^{\rho}\right)^{\beta}\left(I-\Delta(l)^{-1} L\left(l^{-1}\right)\right) \varphi\right\|_{\hat{2}} \leq a\left(|l|^{\prime}\right)^{v} t^{-D^{\prime} / 4} t^{-(|\alpha|+v) / 2} e^{\omega\left(1+\rho^{2}\right) t}\|\varphi\|_{\hat{1}}
$$

for all $\varphi \in C_{c}^{\infty}(G)$ and $l \in G$. Combining these estimates one deduces as in [ElR6] that for all $M>0$ there exist $a>0$ and $\omega \geq 0$ such that

$$
\left\|\left(A^{\rho}\right)^{\alpha} S_{t}^{\rho}\left(\widehat{A}^{\rho}\right)^{\beta} \varphi\right\|_{\infty} \leq a t^{-D^{\prime} / 2} t^{-(|\alpha|+|\beta|) / 2} e^{\omega\left(1+\rho^{2}\right) t}\|\varphi\|_{\hat{i}}
$$

and

$$
\left\|\Delta(l)^{-1} L(k)\left(A^{\rho}\right)^{\alpha} S_{t}^{\rho}\left(\widehat{A}^{\rho}\right)^{\beta} L\left(l^{-1}\right)-\left(A^{\rho}\right)^{\alpha} S_{t}^{\rho}\left(\widehat{A}^{\rho}\right)^{\beta}\right\|_{\hat{\imath} \rightarrow \infty}
$$

$$
\leq a\left(\left(|k|^{\prime} t^{-1 / 2}\right)^{\nu}\left(|l|^{\prime} t^{-1 / 2}\right)^{\nu^{*}}+\left(|k|^{\prime} t^{-1 / 2}\right)^{\nu}+\left(|l|^{\prime} t^{-1 / 2}\right)^{\nu^{*}}\right) t^{-D^{\prime} / 2} t^{-(|\alpha| H|\beta|) / 2} e^{\omega\left(1+\rho^{2}\right) t}
$$

uniformly for all $H \in \mathscr{E}^{\text {div }}\left(N_{2}+v_{2}, N_{1}+v_{1}, N_{1}^{\prime}+v_{1}^{\prime}, N_{0}+v_{0}^{\prime}, \mu, M\right), k, l \in G$, $\alpha \in J_{N}\left(d^{\prime}\right), \beta \in J_{N^{*}}\left(d^{\prime}\right), \rho \in \mathbb{R}$ and $\psi \in C_{c}^{\infty}(G)$ with $\left\|A^{\gamma} \psi\right\|_{\infty} \leq 1$ for all $\gamma \in J\left(d^{\prime}\right)$ with $1 \leq|\gamma| \leq N \vee N^{*} \vee N^{\prime}+1$.

Now it follows as in the proof of [EIR6, Proposition 4.5] that for all $\alpha \in J_{N}\left(d^{\prime}\right)$ and $\beta \in J_{N^{*}}\left(d^{\prime}\right)$ the operator $A^{\alpha} S_{t} \widehat{A}^{\beta}$ has a continuous kernel $K_{t}^{(\alpha, \beta)}$. Of course 
$K_{t}=K^{(\alpha, \beta)}$ is the kernel of $S_{t}$ if $|\alpha|=|\beta|=0$. But for all $\varphi_{1}, \varphi_{2} \in C_{c}^{\infty}(G)$ one has

$$
\begin{aligned}
(-1)^{|\alpha|+|\beta|} \int_{G} d g \int_{G} d \hat{h} K_{t}(g ; h)\left(A^{\alpha} \varphi_{1}\right)(g)\left(\widehat{A}^{\beta} \varphi_{2}\right)(h) \\
=(-1)^{|\alpha|+|\beta|}\left(A^{\alpha} \overline{\varphi_{1}}, S_{t} \widehat{A}^{\beta} \varphi\right)=(-1)^{|\beta|}\left(\overline{\varphi_{1}}, A^{\alpha} \widehat{S}_{t} \widehat{A}^{\beta} \varphi_{2}\right) \\
=(-1)^{|\beta|} \int_{G} d g \varphi_{1}(g)\left(A^{\alpha} S_{t} \widehat{A}^{\beta} \varphi_{2}\right)(g) \\
=(-1)^{|\beta|} \int_{G} d g \int_{G} d \hat{h} K_{t}^{(\alpha, \beta)}(g ; h) \varphi_{1}(g) \varphi_{2}(h) .
\end{aligned}
$$

So by density

$$
(-1)^{|\alpha|+|\beta|} \int_{G} d g \int_{G} d \hat{h} K_{t}(g ; h)\left(A^{\alpha} \widehat{B}^{\beta} \varphi\right)(g ; h)=(-1)^{|\beta|} \int_{G} d g \int_{G} d \hat{h} K_{t}^{(\alpha, \beta)}(g ; h) \varphi(g ; h)
$$

for all $\varphi \in C_{c}^{\infty}(G \times G)$ and the $(-1)^{|\beta|} K_{t}^{(\alpha, \beta)}$ are the successive distributional derivatives of $K_{t}$. Since the $K_{t}^{(\alpha, \beta)}$ are continuous one deduces from the lemma of Du Bois-Reymond that $K_{t}$ is $N$ times differentiable in the first variable, the derivatives are $N^{*}$-times differentiable in the second variable and all derivatives are continuous. Then the bounds (26) give

$$
\left|\left(A^{\alpha} B^{\beta} K_{t}\right)(g ; h)\right| \leq a t^{-D^{\prime} / 2} t^{-(|\alpha|+|\beta|) / 2} e^{\omega\left(1+\rho^{2}\right) t} e^{\rho(\psi(g)-\psi(h))}
$$

for all $g, h \in G$ and minimizing over $\rho$ and $\psi$, using [Rob, pp. 201-202], gives the bounds

$$
\left|\left(A^{\alpha} B^{\beta} K_{t}\right)(g ; h)\right| \leq a t^{-D^{\prime} / 2} t^{-(|\alpha|+|\beta|) / 2} e^{\omega^{\prime} t} e^{-b\left|g h^{-1}\right|^{2} t^{-1}}
$$

where $|\cdot|$ is the strongly elliptic distance. This enters because the estimates involve the second and higher derivatives of the $\psi$. Note that in the strongly elliptic case we have now proved the bounds (22).

Next we use a scaling argument to deduce that the bounds (22) are also valid in the stratified case. For $u>0$ let $\gamma_{u}$ be the dilations on $G$. For $u \in[1, \infty)$ let $H_{u}$ be the operator with coefficients $c_{i j}^{(u)}=c_{i j} \circ \gamma_{u}^{-1}, c_{i}^{(u)}=u^{-1} c_{i} \circ \gamma_{u}^{-1}, c_{i}^{\prime(u)}=u^{-1} c_{i}^{\prime} \circ \gamma_{u}^{-1}$ and $c_{0}^{(u)}=u^{-2} c_{0} \circ \gamma_{u}^{-1}$. Then $H_{u} \in \mathscr{E}^{\text {div }}\left(N_{2}+v_{2}, N_{1}+v_{1}, N_{1}^{\prime}+v_{1}^{\prime}, N_{0}+v_{0}^{\prime}, \mu, M\right)$, uniformly for all $u \geq 1$. Moreover, $\left(H\left(\varphi \circ \gamma_{u}\right)\right) \circ \gamma_{u}^{-1}=u^{2} H_{u} \varphi$ and hence $\left(S_{t}\left(\varphi \circ \gamma_{u}\right)\right) \circ \gamma_{u}^{-1}=$ $S_{u^{2} t}^{(u)} \varphi$, where $S^{(u)}$ is the semigroup generated by $H_{u}$. Therefore the kernel $K^{(u)}$ of the semigroup $S^{(u)}$ satisfies $K_{u^{2} t}^{(u)}(g ; h)=u^{-D^{\prime}} K_{t}\left(\gamma_{u}^{-1}(g) ; \gamma_{u}^{-1}(h)\right)$ for all $t>0$. Consequently, setting $u=t^{-1 / 2}$ one finds

$$
K_{t}(g ; h)=t^{-D^{\prime} / 2} K_{1}^{(u)}\left(\gamma_{t^{-1 / 2}}(g) ; \gamma_{t^{-1 / 2}}(h)\right)
$$


for all $t \in\langle 0,1]$ and $g, h \in G$. Similarly,

$$
\left(A^{\alpha} B^{\beta} K_{t}\right)(g ; h)=t^{-D^{\prime} / 2} t^{-(|\alpha|+|\beta|) / 2}\left(A^{\alpha} B^{\beta} K_{1}^{\left(t^{-1 / 2}\right)}\right)\left(\gamma_{t^{-1 / 2}}(g) ; \gamma_{t^{-1 / 2}}(h)\right)
$$

for all $t \in\langle 0,1]$ and $g, h \in G$.

By [VSC, Proposition III.4.2] there exists an $\eta \geq 1$ such that $|g| \geq \eta|g|^{\prime}$ for all $g \in G$ with $|g|^{\prime} \geq 1$. Then

$$
e^{-b|g|^{2} t^{-1}} \leq e^{b \eta} e^{-b \eta\left(|g|^{\prime}\right)^{2} t^{-1}}
$$

for all $g \in G$ and $t \in[1, \infty)$. Therefore, by (28)

$$
\left|\left(A^{\alpha} B^{\beta} K_{t}^{(u)}\right)(g ; h)\right| \leq a t^{-D^{\prime} / 2} t^{-(|\alpha|+|\beta|) / 2} e^{\omega^{\prime} t} e^{-b\left(\left|g h^{-1}\right|^{\prime}\right)^{2} t^{-1}}
$$

uniformly for all $u \geq 1, t \geq 1$ and $g, h \in G$, for some redefined $a$ and $b$. So for all $t \geq 1$ the estimates (22) follow by setting $u=1$.

If $t \in\langle 0,1]$ a combination with (29) yields

$$
\begin{aligned}
\left|\left(A^{\alpha} B^{\beta} K_{t}\right)(g ; h)\right| & =t^{-D^{\prime} / 2} t^{-(|\alpha|+|\beta|) / 2}\left|\left(A^{\alpha} B^{\beta} K_{1}^{\left(t^{-1 / 2}\right)}\right)(g ; h)\right| \\
& \leq a t^{-D^{\prime} / 2} t^{-(|\alpha|+|\beta|) / 2} e^{-b\left(\left|g h^{-1}\right|^{\prime}\right)^{2} t^{-1}}
\end{aligned}
$$

uniformly for all $t \in\langle 0,1]$ and $g, h \in G$ and one deduces the estimates (22) for the subelliptic operators.

Next we turn to the Hölder bounds. Starting from the semigroup bounds (27) it follows as in [EIR6] that there exist $a, a^{\prime}, b, \omega>0$ such that

$$
\begin{aligned}
& \left|\left(A^{\alpha} B^{\beta} K_{t}\right)(g ; h)-\left(A^{\alpha} B^{\beta} K_{t}\right)\left(k^{-1} g ; l^{-1} h\right)\right| \\
& \leq a\left(\left(|k|^{\prime} t^{-1 / 2}\right)^{\nu}\left(|l|^{\prime} t^{-1 / 2}\right)^{\nu^{*}}+\left(|k|^{\prime} t^{-1 / 2}\right)^{\nu}+\left(|l|^{\prime} t^{-1 / 2}\right)^{v^{*}}\right) t^{-D^{\prime} / 2} t^{-(|\alpha|+|\beta|) / 2} e^{\omega t} e^{-c\left|g h^{-1}\right|^{2} t^{-1}} \\
& \quad+a^{\prime}\left|g h^{-1}\right| t^{-1 / 2}(|k|+|l|) t^{-1 / 2} e^{2 c t^{-1}\left|g h^{-1}\right|(|k|+|l|)} t^{-D^{\prime} / 2} t^{-(|\alpha|+|\beta|) / 2} e^{\omega^{\prime} t} e^{-b\left(\left|k^{-1} g h^{-1} l\right|^{\prime}\right)^{2} t^{-1}}
\end{aligned}
$$

uniformly for all $g, h, k, l \in G, t>0$ and large $\omega$, where $c=(4 \omega)^{-1}$. Note that the strongly elliptic distance enters even in the subelliptic case. By increasing $\omega$ one can make $c$ arbitrarily small. Obviously, $\left|g h^{-1}\right| t^{-1 / 2}(|k|+|l|) t^{-1 / 2} e^{2 c t^{-1}\left|g h^{-1}\right|(|k|+|l|)} \leq\left|g h^{-1}\right|^{\prime} t^{-1 / 2}\left(|k|^{\prime}+|l|^{\prime}\right) t^{-1 / 2} e^{2 c t^{-1}\left|g h^{-1}\right|^{\prime}\left(|k|^{\prime}+|l|^{\prime}\right)}$ since $|g| \leq|g|^{\prime}$. Moreover, $e^{-c\left|g h^{-1}\right|^{2} t^{-1}} \leq e^{c \eta} e^{-c \eta\left(\left|g h^{-1}\right|^{\prime}\right)^{2} t^{-1}}$ by the estimates (30) if $t \in[1, \infty)$.

Now suppose either $H$ is strongly elliptic and $t \in\langle 0, \infty\rangle$ or $G$ is stratified and $t \in[1, \infty)$. Then

$$
\begin{aligned}
& \left|\left(A^{\alpha} B^{\beta} K_{t}\right)(g ; h)-\left(A^{\alpha} B^{\beta} K_{t}\right)\left(k^{-1} g ; l^{-1} h\right)\right| \\
& \leq a\left(\left(|k|^{\prime} t^{-1 / 2}\right)^{v}\left(|l|^{\prime} t^{-1 / 2}\right)^{v^{*}}+\left(|k|^{\prime} t^{-1 / 2}\right)^{\nu}+\left(|l|^{\prime} t^{-1 / 2}\right)^{v^{*}}\right) t^{-D^{\prime} / 2} t^{-(|\alpha|+|\beta|) / 2} e^{\omega t} e^{-c\left(\left|g h^{-1}\right|^{\prime}\right)^{2} t^{-1}} \\
& \quad+a^{\prime}\left|g h^{-1}\right|^{\prime} t^{-1 / 2}\left(|k|^{\prime}+|l|^{\prime}\right) t^{-1 / 2} e^{2 c t^{-1}\left|g h^{-1}\right|^{\prime}\left(|k|^{\prime}+\mid l l^{\prime}\right)} t^{-D^{\prime} / 2} t^{-(|\alpha|+|\beta|) / 2} e^{\omega^{\prime} t} e^{-b\left(\left|k^{-1} g h^{-1} l\right|^{\prime}\right)^{2} t^{-1}}
\end{aligned}
$$


for some redefined constants. One can now continue to argue as in [EIR6] and deduce that for all $M>0$ and $\kappa>0$ there exist $a, b, \omega>0$ such that

$$
\begin{aligned}
& \left|K_{t}(g ; h)-K_{t}\left(k^{-1} g ; l^{-1} h\right)\right| \\
& \quad \leq a t^{-D^{\prime} / 2} e^{\omega t}\left(\left(\frac{|k|^{\prime}}{t^{1 / 2}+\left|g h^{-1}\right|^{\prime}}\right)^{\nu}+\left(\frac{|l|^{\prime}}{t^{1 / 2}+\left|g h^{-1}\right|^{\prime}}\right)^{v^{*}}\right) e^{-b\left(\left|g h^{-1}\right|^{\prime}\right)^{2} t^{-1}},
\end{aligned}
$$

uniformly for all $H \in \mathscr{E}^{\text {div }}\left(N_{2}+\nu_{2}, N_{1}+v_{1}, N_{1}^{\prime}+v_{1}^{\prime}, N_{0}+v_{0}, \mu, M\right)$ and $g, h, k, l \in G$, whenever $|k|^{\prime}+|l|^{\prime} \leq \kappa t^{1 / 2}+2^{-1}\left|g h^{-1}\right|^{\prime}$. This establishes the estimates (23), except in the stratified case if $t \in\langle 0,1\rangle$. But these can be deduced by a scaling argument from the bounds with $t=1$.

We combine Propositions 3.3, 3.4 and 3.5.

THEOREM 3.6. Let $v_{2} \in\langle 0,1\rangle, v_{1}, v_{1}^{\prime} \in\left[0, v_{2}\right]$ and $H$ and subelliptic operator in divergence form (1). Suppose either

(a) $H$ is strongly elliptic, or

(b) $G$ is stratified and $a_{1}, \ldots, a_{d^{\prime}}$ is a basis for $\mathfrak{g}_{1}$ in the stratification $\left(\mathfrak{g}_{m}\right)_{m \in\{1, \ldots, r\}}$ of $\mathrm{g}$.

Let $N, N^{*} \in\{0,1\}, v, v^{*} \in[0,1\rangle$ and suppose that $N+v \leq 1+v_{1}^{\prime}$ and $N^{*}+v^{*} \leq$ $1+v_{1}$ with the exception of the case $N+v=1$ if $v_{1}^{\prime}=0$ and with the exception of the case $N^{*}+v^{*}=1$ if $v_{1}=0$. If $c_{i j} \in C^{v_{2} \prime}, c_{i} \in C^{\nu_{1} \prime}$ if $v_{1}>0, c_{i}^{\prime} \in C^{\nu_{1}^{\prime} '}$ if $v_{1}^{\prime}>0$ and $c_{0} \in L_{\infty}$ then for all $\kappa>0$ there exist $a, b>0$ and $\omega \geq 0$, such that the kernel $K$ of the semigroup $S$ generated by $H$ is $N$-times differentiable in the first variable, the derivatives with respect to the first are $N^{*}$-times differentiable with respect to the second, the derivatives are continuous,

$$
\left|\left(A^{\alpha} B^{\beta} K_{t}\right)(g ; h)\right| \leq a t^{-D^{\prime} / 2} t^{-(|\alpha|+|\beta|) / 2} e^{\omega^{\prime} t} e^{-b\left(\left|g h^{-1}\right|^{\prime}\right)^{2} t^{-1}}
$$

and

$$
\begin{aligned}
& \left|\left(A^{\alpha} B^{\beta} K_{t}\right)\left(k^{-1} g ; l^{-1} h\right)-\left(A^{\alpha} B^{\beta} K_{t}\right)(g ; h)\right| \\
& \quad \leq a t^{-D^{\prime} / 2} t^{-(|\alpha|+|\beta|) / 2} e^{(\omega t}\left(\left(\frac{|k|^{\prime}}{t^{1 / 2}+\left|g h^{-1}\right|^{\prime}}\right)^{v}+\left(\frac{|l|^{\prime}}{t^{1 / 2}+\left|g h^{-1}\right|^{\prime}}\right)^{v^{*}}\right) e^{-b\left(\left|g h^{-1}\right|^{\prime}\right)^{2} t^{-1}}
\end{aligned}
$$

uniformly for all $\alpha \in J_{N}\left(d^{\prime}\right), \beta \in J_{N^{*}}\left(d^{\prime}\right), t>0, g, h \in G$ and $k, l \in G$ such that $|k|^{\prime}+|l|^{\prime} \leq \kappa t^{1 / 2}+2^{-1}\left|g h^{-1}\right|^{\prime}$.

Moreover, for all $M, \mu>0, N, N^{*}, \nu, \nu^{*}$ and $\kappa$ the constants $a, b$ and $\omega$ are uniform for all $H \in \mathscr{E}^{\text {div }}\left(\nu_{2}, v_{1}, v_{1}^{\prime}, 0, \mu, M\right)$

PROOF. This follows immediately from Propositions 3.3, 3.4 and 3.5. 
COROLLARY 3.7. Adopt the hypotheses of Proposition 3.2. If $c_{i j}, c_{i}^{\prime} \in C^{\nu \prime}$ for some $v \in\langle 0,1\rangle$ and $c_{i}, c_{0} \in L_{\infty}$ then $S_{t}$ maps $L_{p}$ into $L_{p: 1}^{\prime}$ and there exist $a>0$ and $\omega \geq 0$ such that

$$
\begin{aligned}
\left\|S_{t}^{\rho} \varphi\right\|_{p} & \leq a e^{\omega\left(1+\rho^{2}\right) t}\|\varphi\|_{p}, \\
\left\|A_{i} S_{t}^{\rho} \varphi\right\|_{p} & \leq a t^{-1 / 2} e^{\omega\left(1+\rho^{2}\right) t}\|\varphi\|_{p}
\end{aligned}
$$

and

$$
\left\|(I-L(k)) A_{i} S_{t} \varphi\right\|_{p} \leq a\left(|k|^{\prime}\right)^{\nu} t^{-(1+v) / 2} e^{\omega t}\|\varphi\|_{p}
$$

uniformly for all $t>0, p \in[1, \infty], i \in\left\{1, \ldots, d^{\prime}\right\}, k \in G, \varphi \in L_{p}, \rho \in \mathbb{R}$ and $\psi \in C_{c}^{\infty}(G)$ with $\left\|A_{j} \psi\right\|_{\infty} \leq 1$ for all $j \in\left\{1, \ldots, d^{\prime}\right\}$. Moreover, for all $M, \mu>0$ the constants $a$ and $\omega$ can be chosen uniform for all $H \in \mathscr{E}^{\text {div }}(\nu, 0, \nu, 0, \mu, M)$.

Similar results are valid on $L_{\hat{p}}$.

PROOF. The operator $U_{\rho} A_{i} S_{t} U_{\rho}^{-1}$ has kernel $(g, h) \mapsto e^{-\rho(\psi(g)-\psi(h))}\left(A_{i} K_{t}\right)(g ; h)$ and

$$
\left|e^{-\rho(\psi(g)-\psi(h))}\left(A_{i} K_{t}\right)(g ; h)\right| \leq a t^{-1 / 2} e^{\omega t} G_{t}^{\rho}\left(g h^{-1}\right)
$$

where $G_{t}^{\rho}(g)=t^{-D^{\prime} / 2} e^{-b\left(|g|^{\prime}\right)^{2} t^{-1}} e^{\left.|\rho \||\right|^{\prime}}$ for some $a, b>0$ and $\omega \geq 0$. So

$$
\begin{aligned}
\left|\left(U_{\rho} A_{i} S_{t} U_{\rho}^{-1} \varphi\right)(g)\right| & \leq \int_{G} d \hat{h}\left|e^{-\rho(\psi(g)-\psi(h))}\left(A_{i} K_{t}\right)(g ; h)\right||\varphi(h)| \\
& \leq \int_{G} d \hat{h} a t^{-1 / 2} e^{\omega t} G_{t}^{\rho}\left(g h^{-1}\right)|\varphi(h)|=a t^{-1 / 2} e^{\omega t}\left(G_{t}^{\rho} *|\varphi|\right)(g) .
\end{aligned}
$$

Therefore

$$
\left\|U_{\rho} A_{i} S_{t} U_{\rho}^{-1} \varphi\right\|_{p} \leq a t^{-1 / 2} e^{\omega t}\left\|G_{t}^{\rho}\right\|_{1}\|\varphi\|_{p} \leq a^{\prime} t^{-1 / 2} e^{\omega^{\prime}\left(1+\rho^{2}\right) t}\|\varphi\|_{p} .
$$

Similarly $\left\|S_{t}^{\rho} \varphi\right\|_{p} \leq a e^{\omega\left(1+\rho^{2}\right) r}\|\varphi\|_{p}$. Then

$$
\left\|A_{i} S_{t}^{\rho} \varphi\right\|_{p}=\left\|U_{\rho} A_{i} S_{t} U_{\rho}^{-1} \varphi-\rho \psi_{i} S_{t}^{\rho} \varphi\right\|_{p} \leq a t^{-1 / 2} e^{\omega\left(1+\rho^{2}\right) t}\|\varphi\|_{p} .
$$

Next we prove the Hölder bounds. If $|k|^{\prime} \geq t^{1 / 2}$ then

$$
\left\|(I-L(k)) A_{i} S_{t} \varphi\right\|_{p} \leq 2\left\|A_{i} S_{t} \varphi\right\|_{p} \leq 2 a t^{-1 / 2} e^{\omega t}\|\varphi\|_{p} \leq 2 a\left(|k|^{\prime}\right)^{v} t^{-(1+\nu) / 2} e^{\omega t}\|\varphi\|_{p} .
$$

Alternatively, if $|k|^{\prime} \leq t^{1 / 2}$ then

$$
\left|\left(A_{i} K_{t}\right)\left(k^{-1} g ; h\right)-\left(A_{i} K_{t}\right)(g ; h)\right| \leq a\left(|k|^{\prime}\right)^{v} t^{-(1+v) / 2} e^{\omega t} G_{t}\left(g h^{-1}\right)
$$

where $G_{t}(g)=t^{-D^{\prime} / 2} e^{-b\left(|g|^{\prime}\right)^{2} t^{-1}}$ for some $a, b>0$ and $\omega \geq 0$. So for all $g \in G$ one has

$$
\left|\left((I-L(k)) A_{i} S_{t} \varphi\right)(g)\right| \leq a\left(|k|^{\prime}\right)^{\nu} t^{-(1+\nu) / 2} e^{\omega t}\left(G_{t} *|\varphi|\right)(g) .
$$


Therefore

$$
\left\|(I-L(k)) A_{i} S_{t} \varphi\right\|_{p} \leq a\left(|k|^{\prime}\right)^{v} t^{-(1+v) / 2} e^{\omega t}\left\|G_{t}\right\|_{1}\|\varphi\|_{p} \leq a^{\prime}\left(|k|^{\prime}\right)^{\nu} t^{-(1+v) / 2} e^{\omega^{\prime} t}\|\varphi\|_{p},
$$

and the corollary follows.

COROLLARY 3.8. Adopt the hypotheses of Proposition 3.2. If $c_{i j}, c_{i}^{\prime} \in C^{\nu \prime}$ for some $v \in\langle 0,1\rangle$ and $c_{i}, c_{0} \in L_{\infty}$ then there exist $\lambda_{0}>0$ and $a>0$ such that $(\lambda I+H)^{-1}$ maps $L_{p}$ into $L_{p: 1}^{\prime}$ and $\left\|A_{i}(\lambda I+H)^{-1}\right\|_{p \rightarrow p} \leq a \lambda^{-1 / 2}$ uniformly for all $\lambda \geq \lambda_{0}$ and $p \in[1, \infty]$. Moreover, for all $M, \mu>0$ the constants $a$ and $\lambda_{0}$ can be chosen uniform for all $H \in \mathscr{E}^{\text {div }}(\nu, 0, \nu, 0, \mu, M)$

Similar results are valid on $L_{\hat{p}}$.

PROOF. This follows from the Laplace transform of the bounds

$$
\left\|A_{i} S_{t} \varphi\right\|_{p} \leq a t^{-1 / 2} e^{\omega t}\|\varphi\|_{p}
$$

of the previous corollary.

COROLLARY 3.9. Let $H$ be a subelliptic operator in divergence form (1). Suppose either

(a) $H$ is strongly elliptic, or

(b) $G$ is stratified and $a_{1}, \ldots, a_{d^{\prime}}$ is a basis for $\mathfrak{g}_{1}$ in the stratification $\left(\mathfrak{g}_{m}\right)_{m \in\{1, \ldots, r\}}$ of $\mathfrak{g}$.

If $c_{i j}, c_{i} \in C^{\nu}$ for some $v \in\langle 0,1\rangle$ and $c_{i}^{\prime}, c_{0} \in L_{\infty}$ then there exist $\lambda_{0}>0$ and $a>0$ such that $(\lambda I+H)^{-1}$ maps $L_{p ;-1}^{\prime}$ into $L_{p}$ and

$$
\left\|(\lambda I+H)^{-1} A_{i}\right\|_{p \rightarrow p} \leq a \lambda^{-1 / 2}
$$

uniformly for all $\lambda \geq \lambda_{0}$ and $p \in[1, \infty]$. Similar results are valid on $L_{\hat{p}}$.

\section{Resolvent estimates}

Let $H$ be a subelliptic operator in divergence form (1). We next examine properties of the resolvents $(\lambda I+H)^{-1}$ acting on the $L_{p}$-spaces, $L_{p}(G ; d g)$, with $p \in\langle 1, \infty\rangle$, and on the associated spaces $L_{\hat{p}}=L_{p}(G ; d \hat{g})$.

The operator $H$ is initially defined on $L_{2}$ through the sectorial form $h$ given by (3) and the resolvents $(\lambda I+H)^{-1}$ do not necessarily extend to the $L_{p}$-spaces or the related Sobolev spaces (see $[\mathrm{ACT}]$ ). This requires some smoothness of the coefficients.

If the principal coefficients of $H$ are right uniformly continuous then $H$ generates a continuous holomorphic semigroup $S$ on $L_{2}$ which extends to all the $L_{p^{-}}$, and 
$L_{\hat{p}^{-}}$, spaces by [ElR6, Theorem 1.1]. The action of $S_{t}$ is determined by a kernel $K_{t}$ which satisfies Gaussian bounds and is Hölder continuous. One cannot expect a Gaussian kernel to exist for general strongly elliptic, complex, operators [ACT]. It is essential that the principal coefficients are real or satisfy some smoothness condition such as uniform continuity. The existence of a kernel satisfying (Hölder continuous) Gaussian bounds does, however, imply many more structural properties of $H$ and $S$. For example, it follows as in [EIR3] using arguments as in the proof of Theorem 4.6 below, that $H$ has a bounded holomorphic functional calculus on each of the $L_{p^{-}}$, or $L_{\hat{p}^{-}}$, spaces with $p \in\langle 1, \infty\rangle$. This in turn implies some domain properties for $H$ and its fractional powers.

If $\lambda>0$ is large enough then $\lambda I+H$ generates an exponentially decreasing semigroup uniformly on all the $L_{p}$-spaces, where $p \in[1, \infty]$. Then the fractional powers $(\lambda I+H)^{\gamma}, \gamma \in[0,1]$, are defined on $L_{p}$. Note that the (graph-)norms on the spaces $D\left(\left(\lambda_{1} I+H\right)^{\gamma}\right)$ and $D\left(\left(\lambda_{2} I+H\right)^{\gamma}\right)$ are equivalent whenever $\lambda_{1}, \lambda_{2} \geq \lambda$ (see [Rob, Lemma II.3.2]). Next the existence of a bounded holomorphic functional calculus implies $D\left((\lambda I+H)^{\gamma}\right)=\left[L_{p}, D(H)\right]_{\gamma}$, for all $\gamma \in\langle 0,1\rangle$, and $D((\lambda I+$ $\left.H)^{\gamma}\right)=\left[L_{p}, D\left((\lambda I+H)^{1 / 2}\right)\right]_{2 \gamma}$, for all $\gamma \in\langle 0,1 / 2\rangle$, where $[\cdot, \cdot]_{\gamma}$ denotes the complex interpolation space. But these identities are not particularly useful in relating the domains with the Sobolev spaces unless one has some further identification of $D(H)$ or $D\left((\lambda I+H)^{1 / 2}\right)$.

The Kato problem consists of establishing conditions under which $D((\lambda I+$ $\left.H)^{1 / 2}\right)=L_{2 ; 1}^{\prime}$. Once this condition is verified one then has $D\left((\lambda I+H)^{\gamma / 2}\right)=L_{2 ; \gamma}^{\prime}$ for $\gamma \in\langle 0,1]$. Note that it follows from [Kat1, Theorem 3.1], that $D\left((\lambda I+H)^{\gamma / 2}\right)=L_{2 ; \gamma}^{\prime}$ for all $\gamma \in\langle 0,1\rangle$ even if the coefficients are only measurable. The difficulty is with the value $\gamma=1$.

If $G=\mathbb{R}^{d}$ and the coefficients are Hölder continuous the Kato problem has been resolved positively by Lions [Lio], McIntosh [McI] and [AMT]. In [McI] domains in $\mathbb{R}^{d}$ are also allowed under some weak smoothness assumptions. On (general) Lie groups these results are slightly weakened.

THEOREM 4.1. Let $H$ be a subelliptic operator in divergence form (1). If $c_{i j} \in C^{\nu \prime}$ for some $v \in\langle 0,1\rangle$ and $c_{i}, c_{i}^{\prime}, c_{0} \in L_{\infty}$ then $D\left((\lambda I+H)^{1 / 2}\right)=L_{2 ; 1}^{\prime}$ for all large $\lambda$.

Moreover, for all $M, \mu>0$ there exists a $\lambda_{0}>0$ such that for all $\gamma \in\langle 0, \nu\rangle$ and $\lambda \geq \lambda_{0}$ there exists an $a>0$ such that $L_{2 ; 1+\gamma}^{\prime} \subseteq D\left((\lambda I+H)^{(1+\gamma) / 2}\right)$ and

$$
\left\|(\lambda I+H)^{(1+\gamma) / 2} \varphi\right\|_{2} \leq a\|\varphi\|_{2 ; 1+\gamma}^{\prime}
$$

uniformly for all $\varphi \in L_{2 ; 1+\gamma}^{\prime}$ and for all operators $H$ with $\mu_{C} \geq \mu,\|C\|_{C^{\prime}} \leq M$, $\|C\|_{\infty} \leq M,\|c\|_{\infty} \leq M$ and $\left\|c_{0}\right\|_{\infty} \leq M$.

Similar conclusions are valid on $L_{\hat{2}}$. 
PROOF. This theorem is basically proved in [ElR5]. The lower order terms $\sum A_{i} c_{i}^{\prime}+$ $c_{0} I$ can be added directly to the principal part in [EIR5] and then the lower order terms $\sum c_{i} A_{i}$ can be added by the perturbation-interpolation argument. The uniformity of the constants follows from the proof, together with the next lemma.

LEMMA 4.2. For all $M, \mu>0$ there exists $a \lambda_{0}>0$ such that for all $\gamma \in\langle 0,1\rangle$ there exists an $a>0$ such that for all subelliptic operators $H$ of the form (1) with $\mu_{C} \geq \mu$, $\|C\|_{\infty} \leq M,\|c\|_{\infty} \leq M$ and $\left\|c_{0}\right\|_{\infty} \leq M$ one has $D\left((\lambda I+H)^{\gamma}\right)=\left[L_{2}, D(\lambda I+H)\right]_{\gamma}$ and

$$
a^{-1}\|\varphi\|_{\left[L_{2}, D(\lambda I+H)\right]_{Y}} \leq\left\|(\lambda I+H)^{\gamma} \varphi\right\|_{2} \leq a\|\varphi\|_{\left[L_{2}, D(\lambda I+H)\right]_{\gamma}}
$$

uniformly for all $\lambda \geq \lambda_{0}$ and $\varphi \in D\left((\lambda I+H)^{\gamma}\right)$. Moreover,

$$
\left\|\left(\lambda_{0} I+H\right)^{\gamma} \varphi\right\|_{2} \leq\left\|(\lambda I+H)^{\gamma} \varphi\right\|_{2}
$$

for all $\lambda \geq \lambda_{0}$ and $\varphi \in D\left(\left(\lambda_{0} I+H\right)^{\gamma}\right)$.

PROOF. Let $\lambda_{0} \in \mathbb{R}$ be such that $\left(\lambda_{0}-1\right) I+H$ is a maximal accretive operator. The constant $\lambda_{0}$ depends only on $M$ and $\mu$. Then for all $\lambda \geq \lambda_{0}$ the operator $\lambda I+H$ is an injective closed maximal accretive operator. So by [ADM, Theorem G], the operator $\lambda I+H$ has a bounded $H_{\infty}(\Lambda(\theta))$-functional calculus, in the sense of [CDMY], for any $\theta \in\langle 0, \pi / 2\rangle$, and

$$
\|f(\lambda I+H)\|_{2 \rightarrow 2} \leq \sup \{|f(z)|: z \in \Lambda(\theta)\}
$$

for all $f \in H_{\infty}(\Lambda(\theta))$. Therefore the operator $\lambda I+H$ has bounded imaginary powers and $\left\|(\lambda I+H)^{i t}\right\|_{2 \rightarrow 2} \leq e^{\pi|t| / 2}$ for all $t \in \mathbb{R}$. Then the first part of the lemma follows from [Tri, Theorem 1.15.3].

Finally, applying the bounded $H_{\infty}(\Lambda(\theta))$-functional calculus of the operator $\lambda_{0} I+$ $H$ to the function $f(z)=z^{(1+\gamma) / 2}\left(\lambda-\lambda_{0}+z\right)^{-(1+\gamma) / 2}$ yields

$\left\|\left(\lambda_{0} I+H\right)^{(1+\gamma) / 2} \varphi\right\|_{2} \leq\left\|f\left(\lambda_{0} I+H\right)\right\|_{2 \rightarrow 2}\left\|(\lambda I+H)^{(1+\gamma) / 2} \varphi\right\|_{2} \leq\left\|(\lambda I+H)^{(1+\gamma) / 2} \varphi\right\|_{2}$ for all $\lambda \geq \lambda_{0}$ and $\varphi \in D\left((\lambda I+H)^{(1+\gamma) / 2}\right)$.

COROLlARY 4.3. For all $M, \mu>0$ there exists $a \lambda_{0}>0$ such that for all $\delta \in$ $\langle 0,1+\nu\rangle$ and $\lambda \geq \lambda_{0}$ there exists an $a>0$ such that $L_{2 ; \delta}^{\prime} \subseteq D\left((\lambda I+H)^{\delta / 2}\right)$ and

$$
\left\|(\lambda I+H)^{\delta / 2} \varphi\right\|_{2} \leq a\|\varphi\|_{2 ; \delta}^{\prime}
$$

uniformly for all $\varphi \in L_{2 ; \delta}^{\prime}$ and for all operators $H$ in divergence form (1) with $\mu_{C} \geq \mu$, $\|C\|_{C^{\prime \prime}} \leq M,\|C\|_{\infty} \leq M,\|c\|_{\infty} \leq M$ and $\left\|c_{0}\right\|_{\infty} \leq M$.

Similar conclusions are valid on $L_{\hat{2}}$. 
It follows from Theorem 4.1 that $L_{2 ; \delta}^{\prime} \subseteq D\left((\lambda I+H)^{\delta / 2}\right)$ on a general Lie group if $c_{i j} \in C^{\nu \prime}$ and $c_{i}, c_{i}^{\prime}, c_{0} \in L_{\infty}$. We next show that the spaces $L_{2 ; \delta}^{\prime}$ and $D\left((\lambda I+H)^{\delta / 2}\right)$ are equal, if, in addition, the $c_{i}^{\prime}$ are Hölder continuous and the operator is strongly elliptic or the group is stratified, by exploitation of the heat kernel bounds developed in Section 3.

THEOREM 4.4. Let $H$ be a subelliptic operator in divergence form (1). Suppose either

(a) $H$ is strongly elliptic, or

(b) $G$ is stratified and $a_{1}, \ldots, a_{d^{\prime}}$ is a basis for $\mathfrak{g}_{1}$ in the stratification $\left(\mathfrak{g}_{m}\right)_{m \in\{1, \ldots, r\}}$ of $\mathfrak{g}$.

If $v \in\langle 0,1\rangle, \gamma \in\langle 0, v\rangle, c_{i j}, c_{i}^{\prime} \in C^{\nu \prime}$, and $c_{i}, c_{0} \in L_{\infty}$ then $D\left((\lambda I+H)^{(1+\gamma) / 2}\right) \subseteq$ $L_{2: 1+\gamma}^{\prime}$ for large $\lambda$ and the embedding is continuous.

Moreover, for all $M, \mu>0$ there exists a $\lambda_{0}>0$ such that for all $\gamma \in\langle 0, \nu\rangle$ there exists an $a>0$ such that

$$
\left.\|\varphi\|_{2: 1+\gamma}^{s} \leq a \|\langle\lambda\rangle+H\right)^{\left(1+5 \zeta \zeta S_{2}\right.} \varphi \|_{2}
$$

uniformly for all $H \in \mathscr{E}^{\text {div }}(\nu, 0, \nu, 0, \mu, M), \lambda \geq \lambda_{0}$ and $\varphi \in D\left((\lambda I+H)^{(1+\gamma) / 2}\right)$.

Similar conclusions are valid on $L_{\hat{2}}$.

PROOF. The proof consists of several steps.

STEP 1. First we reintroduce the Lipschitz spaces associated with left translations on $L_{2}$ of [EIR1], but now we clearly indicate the $p$-dependence in the notation. Let $\mathscr{O}$ be a fixed bounded open neighbourhood of the identity of $G$. For $p \in[1, \infty]$ and $\gamma \in\langle 0,1\rangle$ define $\|\cdot\|_{\gamma}^{L, p}: L_{2} \rightarrow[0, \infty]$ by

$$
\|\varphi\|_{\gamma}^{L, p}=\|\varphi\|_{2}+\left(\int_{0} d g\left(|g|^{\prime}\right)^{-D^{\prime}}\left(\left(|g|^{\prime}\right)^{-\gamma}\|(I-L(g)) \varphi\|_{2}\right)^{p}\right)^{1 / p}
$$

if $p<\infty$ with the obvious modification if $p=\infty$. Then define the Lipschitz space $L_{2 ; \gamma}^{(p)}(L)=\left\{\varphi \in L_{2}(G):\|\varphi\|_{\gamma}^{L, p}<\infty\right\}$. It follows from Corollary 3.7 that $A_{i} S_{t} \varphi \in L_{2 ; \gamma}^{(\infty)}(L)$ and

$$
\left\|A_{i} S_{t} \varphi\right\|_{\gamma}^{L, \infty} \leq a_{\gamma} t^{-(1+\gamma) / 2} e^{\omega_{\gamma} t}\|\varphi\|_{2}
$$

for all $\gamma \in\langle 0, \nu]$ and $\varphi \in L_{2}$. The constants $a_{\gamma}$ and $\omega_{\gamma}$ depend on $H$ through $M$ and $\mu$.

STEP 2. For any interpolation pair $(\mathscr{X}, \mathscr{Y}), \gamma \in\langle 0,1\rangle$ and $p \in[1, \infty]$ let $(\mathscr{X}, \mathscr{Y})_{\gamma, p ; K}$ be the interpolation space by the K-method of Peetre (see [BuB, Definition 3.2.4]). Then it follows from [EIR1, Theorem 3.2], that $L_{2 ; \gamma}^{(p)}(L)=\left(L_{2}, L_{2 ; 1}^{\prime}\right)_{\gamma, p ; K}$, with equivalent norms. Therefore

$$
\left\|A_{i} S_{t} \varphi\right\|_{\left(L_{2}, L_{2: 1}^{\prime}\right)_{\gamma, x: K}} \leq a_{\gamma} t^{-(1+\gamma) / 2} e^{\omega_{\gamma} t}\|\varphi\|_{2}
$$


for all $\gamma \in\langle 0, v]$, and some redefined value of $a_{\gamma}$. But this means that $S_{t}$ maps $L_{2}$ continuously into $\left(L_{2}, L_{2 ; 1}^{\prime}\right)_{\gamma, p ; K ; 1}^{\prime}$, the once-differentiable functions with derivatives in the Lipschitz space. But $\left(L_{2}, L_{2 ; 1}^{\prime}\right)_{\gamma, p ; K ; 1}=\left(L_{2}, L_{2 ; 2}^{\prime}\right)_{(1+\gamma) / 2, p ; K}$ by [EIR1], Theorem 2.1.III, with equivalent norms. Hence

$$
\left\|S_{t} \varphi\right\|_{\left(L_{2}, L_{2: 2}^{\prime}\right)(1+\gamma) / 2 . \infty: K} \leq a_{\gamma} t^{-(1+\gamma) / 2} e^{\omega_{\gamma} t}\|\varphi\|_{2}
$$

for all $\gamma \in\langle 0, \nu]$, for some redefined $a_{y}$. Then, by the reiteration theorem ([BuB, Theorem 3.2.20 and Corollary 3.2.17]), one can deduce information about the interpolation spaces with $p<\infty$. In particular the foregoing bounds can be transferred to the spaces $\left(L_{2}, L_{2 ; 2}^{\prime}\right)_{(1+\gamma) / 2.2: K}$. One deduces that

$$
\left\|S_{t} \varphi\right\|_{\left(L_{2}, L_{2: 2}^{\prime}\right)_{(1+\gamma) / 2,2: K}} \leq a_{\gamma}^{\prime} t^{-(1+\gamma) / 2} e^{\omega_{\gamma}^{\prime} t}\|\varphi\|_{2}
$$

for all $\gamma \in\langle 0, v\rangle$.

STEP 3. Now fix $\gamma \in\langle 0, v\rangle$. We shall prove that

$$
\left(L_{2}, D(H)\right)_{(1+\gamma) / 2,2 ; K} \subseteq\left(L_{2}, L_{2 ; 2}^{\prime}\right)_{(1+\gamma) / 2,2 ; K}
$$

and the inclusion is continuous. Let $\delta \in\langle(1+\gamma) / 2,(1+\nu) / 2\rangle \subset\left\langle 2^{-1}, 1\right\rangle$ and set $\theta=(2 \delta)^{-1}(1+\gamma) \in\langle 0,1\rangle$. By the reiteration theorem it suffices to prove that

$$
\left(L_{2}, D(H)\right)_{(1+y) / 2,2: K} \subseteq\left(L_{2},\left(L_{2}, L_{2 ; 2}^{\prime}\right)_{\delta, 2 ; K}\right)_{\theta, 2: K}
$$

with continuous embedding. Now each $\varphi \in L_{2}$ can be decomposed as $\varphi=(I-$ $\left.S_{t^{\alpha}}\right) \varphi+S_{t^{\alpha}} \varphi$, where $\alpha=\delta^{-1}$. Then one can argue as in Step 4 of the proof of [EIR1, Theorem 3.2], using the bounds of the present Step 2 instead of [EIR 1, Lemma 3.3], that

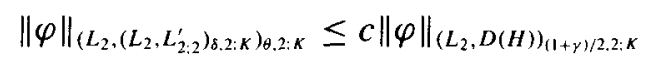

for some $c>0$, independent of $\varphi$, but dependent on $\gamma$ and $H$ through $M$ and $\mu$. Then (32) follows.

STEP 4. The spaces $\left(L_{2}, D(H)\right)_{(1+\gamma) / 2.2: K}$ and $\left(L_{2}, D(|H|)\right)_{(1+\gamma) / 2,2 ; K}$ are equal, with equal norms. Next, for all $\theta \in\langle 0,1\rangle$ there exists an $a\rangle 0$ such that $\left(L_{2}, D(T)\right)_{\theta, 2 ; K}=D\left(T^{\theta}\right)$ and

$$
a^{-1}\|\varphi\|_{\left(L_{2}, D(T) \eta_{\theta, 2: K}\right.} \leq\left\|T^{\theta} \varphi\right\|_{2} \leq a\|\varphi\|_{\left(L_{2}, D(T)\right)_{\theta, 2: \kappa}}
$$

for any positive self-adjoint operator $T$ and all $\varphi \in D\left(T^{\theta}\right)$ (see [LiM, Theorem I.15.1]). But $D\left(T^{\theta}\right)=\left[L_{2}, D(T)\right]_{\theta}$, with equivalent norms, the norms are independent of $T$ (see [Tri, Theorem 1.15.3]). Finally, $\left[L_{2}, D(|H|)\right]_{\theta}=\left[L_{2}, D(H)\right]_{\theta}$, 
with equal norms. So if $\lambda_{0}$ is as in Lemma 4.2, then $\left(L_{2}, D(H)\right)_{(1+\gamma) / 2,2 ; K}=$ $\left[L_{2}, D(H)\right]_{(1+\gamma) / 2}=D\left(\left(\lambda_{0}+H\right)^{(1+\gamma) / 2}\right)$, with equivalent norms, independent of the operator. Repeating these estimates for the Laplacian $H_{L}$ and using the equality $L_{2 ; 2}^{\prime}=D\left(H_{L}\right)$ it follows that there exists an $a>0$, depending only on $\gamma, M$ and $\mu$, such that

$$
\|\varphi\|_{2 ; 1+\gamma}^{\prime} \leq a\left\|\left(\lambda_{0}+H\right)^{(1+\gamma) / 2} \varphi\right\|_{2}
$$

uniformly for all $\varphi \in L_{2 ; 1+\gamma}^{\prime}$.

Finally the bounds uniformly for all $\lambda \geq \lambda_{0}$ follow from inequality (31) of Lemma 4.2.

COROLlaRY 4.5. Adopt the assumptions of Theorem 4.4. For all $v \in\langle 0,1\rangle$, $M, \mu>0$ there exists $a \lambda_{0}>0$ such that for all $\delta \in[0,1+v\rangle$ and $\lambda \geq \lambda_{0}$ there exists an $a>0$ such that $D\left((\lambda I+H)^{\delta / 2}\right)=L_{2 ; \delta}^{\prime}$ and

$$
a^{-1}\left\|(\lambda I+H)^{\delta / 2} \varphi\right\|_{2} \leq\|\varphi\|_{2 ; \delta}^{\prime} \leq a\left\|(\lambda I+H)^{\delta / 2} \varphi\right\|_{2}
$$

uniformly for all $H \in \mathscr{E}^{\text {div }}(\nu, 0, \nu, 0, \mu, M)$ and $\varphi \in L_{2: \delta}^{\prime}$.

Similar conclusions are valid on the $L_{\hat{2}}$-spaces.

Next we extend the Kato regularity to other $L_{p}$-spaces.

THEOREM 4.6. Let $H$ be a subelliptic operator in divergence form (1). Suppose either

(a) $H$ is strongly elliptic, or

(b) $G$ is stratified and $a_{1}, \ldots, a_{d^{\prime}}$ is a basis for $\mathfrak{g}_{1}$ in the stratification $\left(\mathfrak{g}_{m}\right)_{m \in\{1, \ldots, r]}$ of $\mathbf{g}$.

If $c_{i j}, c_{i}^{\prime} \in C^{\nu \prime}$ for some $v \in\langle 0,1\rangle$ and if $p \in\langle 1, \infty\rangle$ then $D\left((\lambda I+H)^{1 / 2}\right) \subseteq L_{p: 1}^{\prime}$ for all large $\lambda$. Moreover, for all $M, \mu>0$ there exists $a \lambda_{0}>0$ such that for all $p \in\langle 1, \infty\rangle$ there exists an $a>0$ such that

$$
\|\varphi\|_{p ; 1}^{\prime} \leq a\left\|(\lambda I+H)^{1 / 2} \varphi\right\|_{p}
$$

uniformly for all $H \in \mathscr{E}^{\text {div }}(\nu, 0, \nu, 0, \mu, M), \lambda \geq \lambda_{0}$ and $\varphi \in D\left((\lambda I+H)^{1 / 2}\right)$.

Similar conclusions are valid on the $L_{\hat{p}}$-spaces.

PROOF. The proof is nearly the same as that of [BER, Theorem 2.3], so we only indicate the significant differences. We prove the embeddings and inclusions on the spaces with the right Haar measure. They then follow for the left Haar measure by the arguments given in [BER, Lemma 2.1].

It suffices to prove the bounds for one particular $\lambda$ since the uniform bounds then follow by the argument used in Step 4 of the proof of Theorem 4.4. This latter 
argument relies on the fact that there exists a $\lambda>0$ such that $\lambda I+H$ has a bounded $H_{\infty}$-functional calculus on the $L_{p}$-spaces uniformly for $p \in\langle 1, \infty\rangle$. If $p=2$ this follows because $\lambda I+H$ is maximal accretive (see [ADM, Theorem G]) and for the other values of $p$ it follows from a similar adaptation of the proof of [EIR3, Theorem 3.1] as the present proof is an adaptation of the proof of [BER].

We may assume that $H$ generates an exponentially decreasing semigroup uniformly on all the $L_{p}$-spaces. Fix $N \in \mathbb{N}, N>D^{\prime}$ and fix a large $\lambda>0$. It will be clear from the proof which value of $\lambda$ is required. For $j \in \mathbb{N}$ consider the operators

$$
X_{j}=j^{N}(j I+H)^{-N}(\lambda I+H)^{-1 / 2} .
$$

Then for fixed $i_{0} \in\left\{1, \ldots, d^{\prime}\right\}$

$$
\left\|A_{i_{0}} X_{j} \varphi\right\|_{\hat{2}} \leq c\left\|(\lambda I+H)^{1 / 2} X_{j} \varphi\right\|_{\hat{2}}=c j^{N}\left\|(j I+H)^{-N} \varphi\right\|_{\hat{2}} \leq c^{\prime}\|\varphi\|_{\hat{2}}
$$

uniformly for all $j \in \mathbb{N}$ and $\varphi \in L_{\hat{2}}$ by the bounds of Theorem 4.4. Thus the operators $A_{i} X_{j}$ are uniformly bounded on $L_{\hat{2}}$.

It follows, as in [BER], that the $X_{j}$ have 'good' kernels, but now they are functions of two variables,

$$
\left(X_{j} \varphi\right)(g)=\int_{G} d \hat{h} k_{j}(g ; h) \varphi(h),
$$

where $k_{j}: G \backslash\{e\} \rightarrow \mathbb{C}$ is defined by $k_{j}(g ; h)=\int_{0}^{\infty} d t f_{j}(t) K_{t}(g ; h)$ and $f_{j}:\langle 0, \infty\rangle \rightarrow$ $\mathbb{R}$ is a function such that

$$
f_{j}(t) \leq a t^{-1 / 2}(j t)^{\tau} e^{-\lambda t}
$$

for some $a>0$, uniformly for all $t>0, \lambda>0, \tau \in[0, N]$ and $j \in \mathbb{N}$ with $j \geq 2 \lambda$ (see [BER, Lemma 2.4]). Choosing $\tau=D^{\prime}$ and using the Gaussian bounds of Theorem 3.6 together with the estimates in the Appendix of [EIR3] one can then show that $k_{j}$ is differentiable in the first variable,

$$
\left(A_{i_{0}} k_{j}\right)(g ; h)=\int_{0}^{\infty} d t f_{j}(t)\left(A_{i_{0}} K_{t}\right)(g ; h)
$$

and

$$
\left|\left(A_{i_{0}} k_{j}\right)(g ; h)\right| \leq c_{j} e^{-b\left(\left|g h^{-1}\right|^{\prime}\right)^{2} t^{-1}}
$$

if $\lambda$ is large enough. In particular the bounds show that the action of $X_{j}$ can be defined with the kernel $k_{j}$ on all the $L_{\hat{p}}$-spaces, and in particular on $L_{\hat{1}}$, for all large $\lambda$. These bounds depend on $j$. Similar bounds starting from $\tau=0$ give $j$ independent bounds for the $A_{i_{0}} k_{j}$,

$$
\left|\left(A_{i_{0}} k_{j}\right)(g ; h)\right| \leq c\left(\left|g h^{-1}\right|^{\prime}\right)^{-D^{\prime}} e^{-b\left(\left|g h^{-1}\right|^{\prime}\right)^{2} t^{-1}}
$$


if $\lambda$ is large enough. But the integral of the latter bounds with respect to left or right Haar measure is logarithmically divergent. Therefore one has to apply the methods of singular integration theory, for example [CoW, Theorem III.2.4], to obtain $L_{\hat{1}^{-}}$ boundedness properties of $A_{i_{0}} X_{j}$.

At this point a problem occurs. The usual theory of singular integrals is restricted to spaces of homogeneous type, for example, spaces with the doubling property. This is sufficient to deal with operators on compact Lie groups or Lie groups of polynomial type but it is not adequate for Lie groups whose volume grows exponentially. In particular it does not apply to non-unimodular groups. For groups of exponential growth one needs a supplementary argument which was first given in [Bur] and later used in [BER] and [DuR].

The starting point is the observation that each Lie group $G$ has a local doubling property. Therefore one can use the estimates in [CoW] on any bounded subset of $G$, whether or not this subset has the doubling property. In the appendix we explain how one can adapt the results of [CoW] to this more general situation.

Because of the bounds (34) we localize the problem as in [BER]. Let $\chi, \chi_{1} \in$ $C_{c}^{\infty}\left(B^{\prime}(2)\right)$ be such that $\chi(g)=\chi_{1}(g)=1$ for all $g \in B^{\prime}(1)$. Consider the operator $T_{j}: L_{\hat{p}}(G) \rightarrow L_{\hat{p}}(G)$ defined by

$$
\left(T_{j} \varphi\right)(g)=\int_{G} d \hat{h} \kappa_{j}(g ; h) \varphi(h)
$$

where

$$
\kappa_{j}(g ; h)=\left(A_{i_{0}} k_{j}\right)(g ; h) \chi\left(g h^{-1}\right) \chi_{1}(h)=\int_{0}^{\infty} d t f_{j}(t)\left(A_{i_{0}} K_{t}\right)(g ; h) \chi\left(g h^{-1}\right) \chi_{1}(h) .
$$

So $\kappa_{j}(g ; h)=0$ if $g \notin B^{\prime}(4)$ or $h \notin B^{\prime}(4)$. Obviously $\kappa_{j} \in L_{2}(G \times G, d \hat{g} \otimes d \hat{h})$ and by the arguments [BER] there exists a $c>0$ such that $\left\|T_{j}\right\|_{\hat{2} \rightarrow \hat{2}} \leq c$ uniformly for all $j \in \mathbb{N}$. These are the first two conditions of the theorem of [CoW] (see the appendix).

For the third and most difficult condition, it suffices to prove that

$$
\sup _{j} \sup _{h, h_{0} \in G} \int_{\Omega\left(h, h_{0}\right)} d \hat{g}\left|\kappa_{j}(g ; h)-\kappa_{j}\left(g ; h_{0}\right)\right|<\infty
$$

where $\Omega\left(h, h_{0}\right)=\left\{g \in G: d^{\prime}\left(g ; h_{0}\right)>4 d^{\prime}\left(h ; h_{0}\right)\right\}$ with $d^{\prime}(\cdot ; \cdot)$ the subelliptic distance on $G$. One deduces from the triangle inequality that the integral is zero if $h \notin B^{\prime}(6)$ or $h_{0} \in B^{\prime}(20 / 3)$.

Then by right invariance

$$
\begin{aligned}
\sup _{j} & \sup _{h . h_{0} \in G} \int_{\Omega\left(h, h_{0}\right)} d \hat{g}\left|\kappa_{j}(g ; h)-\kappa_{j}\left(g ; h_{0}\right)\right| \\
& \leq \sup _{j} \sup _{h, h_{0} \in G} \int_{\Omega_{1}\left(h, h_{0}\right)} d \mu(g)\left|\kappa_{j}\left(g h_{0} ; h\right)-\kappa_{j}\left(g h_{0} ; h_{0}\right)\right|,
\end{aligned}
$$


where $\Omega_{1}\left(h, h_{0}\right)=\left\{g \in B^{\prime}(11):|g|^{\prime}>4\left|h h_{0}^{-1}\right|^{\prime}\right\}$.

Let $h \in B^{\prime}(6), h_{0} \in B^{\prime}(7), g \in B^{\prime}(11)$ and suppose that $|g|^{\prime}>4\left|h h_{0}^{-1}\right|^{\prime}>0$. By the definition of $\kappa_{j}$ we estimate

$$
\begin{gathered}
\left|\left(A_{i_{0}} K_{t}\right)\left(g h_{0} ; h\right) \chi\left(g h_{0} h^{-1}\right) \chi_{1}(h)-\left(A_{i_{0}} K_{t}\right)\left(g h_{0} ; h_{0}\right) \chi\left(g h_{0} h_{0}^{-1}\right) \chi_{1}\left(h_{0}\right)\right| \\
\leq\left|\left(A_{i_{0}} K_{t}\right)\left(g h_{0} ; h\right)-\left(A_{i_{0}} K_{t}\right)\left(g h_{0} ; h_{0}\right)\right|\left|\chi\left(g h_{0} h^{-1}\right)\right|\left|\chi_{1}(h)\right| \\
\quad+\left|\left(A_{i_{0}} K_{t}\right)\left(g h_{0} ; h_{0}\right)\right|\left|\chi\left(g h_{0} h^{-1}\right)-\chi(g) \| \chi_{1}(h)\right| \\
\quad+\left|\left(A_{i_{0}} K_{t}\right)\left(g h_{0} ; h_{0}\right) \| \chi(g)\right|\left|\chi_{1}(h)-\chi_{1}\left(h_{0}\right)\right| \\
\leq a^{\prime}\left(\left|h h_{0}\right|^{\prime}\right)^{v} t^{-D^{\prime} / 2} t^{-(1+\nu) / 2} e^{-b\left(|g|^{\prime}\right)^{2} t^{-1}} e^{(\omega-\lambda) t}
\end{gathered}
$$

by the bounds of Theorem 3.6. These can be applied to the first term since $\left|h h_{0}^{-1}\right|^{\prime} \leq$ $4^{-1}|g|^{\prime}=4^{-1}\left|g h_{0} h_{0}^{-1}\right|^{\prime}$ and for the last two terms one estimates the differences of the functions $\chi$ and $\chi_{1}$ in terms of their derivatives. Using the bounds (33) and estimating as in the Appendix of [ElR3] one deduces that

$$
\begin{aligned}
\left|\kappa_{j}\left(g h_{0} ; h\right)-\kappa_{j}\left(g h_{0} ; h_{0}\right)\right| & \leq \int_{0}^{\infty} d t a t^{-1 / 2} e^{-\lambda t} a^{\prime}\left(\left|h h_{0}\right|^{\prime}\right)^{\nu} t^{-D^{\prime} / 2} t^{-(1+v) / 2} e^{-b\left(|g|^{\prime}\right)^{2} t^{-1}} e^{(\omega-\lambda) t} \\
& \leq a^{\prime \prime}\left(|g|^{\prime}\right)^{-D^{\prime}-v}\left(\left|h h_{0}\right|^{\prime}\right)^{v}
\end{aligned}
$$

uniformly for all $j \geq 2 \lambda$, if $\lambda$ is large enough. But if $c=\sup _{t \in\{0,11]} t^{-D^{\prime}}\left|B_{t}^{\prime}\right|$, $s=d^{\prime}\left(h ; h_{0}\right)=\left|h h_{0}^{-1}\right|^{\prime}$ and $N_{s} \in \mathbb{N}_{0}$ is such that $2^{N_{s}-4} \leq s^{-1} \leq 2^{N_{s}-3}$ then we obtain

$$
\begin{aligned}
\int_{B^{\prime}(11) \backslash B^{\prime}(2 s)} d \hat{g} s^{\nu}\left(|g|^{\prime}\right)^{-D^{\prime}-\nu} & \leq \sum_{n=0}^{N_{s}} \int_{B^{\prime}\left(2^{-n+4}\right) \backslash B^{\prime}\left(2^{-n+3}\right)} d \hat{g} s^{\nu}\left(|g|^{\prime}\right)^{-D^{\prime}-\nu} \\
& \leq \sum_{n=0}^{N_{s}} c s^{\nu}\left(2^{-n+3}\right)^{-D^{\prime}-v}\left(2^{-n+4}\right)^{D^{\prime}} \\
& =2^{D^{\prime}-3 v} c s^{\nu}\left(2^{\nu}-1\right)^{-1}\left(2^{\left(N_{s}+1\right) v}-1\right) \leq 2^{D^{\prime}+v}\left(2^{\nu}-1\right)^{-1} c .
\end{aligned}
$$

Hence

$$
\left.\left.\int_{\Omega_{1}\left(h . h_{0}\right)} d \hat{g} \mid \kappa_{j}\left(g h_{0} ; h\right)\right)-\kappa_{j}\left(g h_{0} ; h_{0}\right)\right) \mid \leq 2^{D^{\prime}+\nu}\left(2^{\nu}-1\right)^{-1} a^{\prime \prime},
$$

which is the third and last condition of Theorem $\mathrm{A} 1$ in the appendix, uniform in $j$.

So the operators $T_{j}$ satisfy uniform weak- $L_{\hat{\imath}}$ estimates and arguing as in Theorem 2.3 in [BER] we obtain the estimates

$$
\rho\left(\left\{g \in G:\left|\left(P_{j} \varphi\right)(g)\right|>\gamma\right\}\right) \leq M_{0} \gamma^{-1}\|\varphi\|_{\hat{1}}
$$

uniformly for $j \in \mathbb{N}$ with $j \geq 2 \lambda$, for $\gamma>0$ and $\varphi \in L_{1}\left(B^{\prime}(1)\right) \cap L_{2}\left(B^{\prime}(1)\right)$, where

$$
\left(P_{j} \varphi\right)(g)=\int_{G} d \hat{h}\left(A_{i_{0}} k_{j}\right)(g ; h) \chi\left(g h^{-1}\right) \varphi(h)
$$


and $\rho$ is the right Haar measure. The value of $M_{0}$ can be chosen uniformly for all $H \in \mathscr{E}^{\text {div }}(\nu, 0, \nu, 0, \mu, M)$ for each prescribed $M>0$.

By [BER, Lemma 2.5] there exist $g_{1}, g_{2}, \ldots \in G$ and $N_{0} \in \mathbb{N}$ such that $G=$ $\bigcup_{i=1}^{\infty} B^{\prime}(1) g_{i}$ and each $g \in G$ is an element of at most $N_{0}$ balls $B^{\prime}(3) g_{i}$. Let $\left(\psi_{i}\right)_{i}$ be a partition of unity relative to the cover $G=\bigcup_{i=1}^{\infty} B^{\prime}(1) g_{i}$. Then supp $P_{j}\left(\psi_{i} \varphi\right) \subseteq$ $B^{\prime}(3) g_{i}$ for all $\varphi \in L_{\hat{1}}(G)$ and $i, j \in \mathbb{N}$ and

$$
\begin{aligned}
\rho(\{g & \left.\left.\in G:\left|\left(P_{j} \varphi\right)(g)\right|>\gamma\right\}\right) \\
& \leq \sum_{i=1}^{\infty} \rho\left(\left\{g \in G:\left|\left(P_{j} R_{g_{i}^{-1}} R_{g_{i}}\left(\psi_{i} \varphi\right)\right)(g)\right|>\gamma N_{0}^{-1}\right\}\right) \\
& =\sum_{i=1}^{\infty} \rho\left(\left\{g \in G:\left|\left(R_{g_{i}} P_{j} R_{g_{i}^{-1}} R_{g_{i}}\left(\psi_{i} \varphi\right)\right)(g)\right|>\gamma N_{0}^{-1}\right\}\right)
\end{aligned}
$$

if $j \geq 2 \lambda$. But if $k \in G$ and $\varphi \in L_{\hat{p}}$ then

$$
\left(R_{k} P_{j} R_{k^{-1}} \varphi\right)(g)=\int_{G} d \hat{h}\left(A_{i_{0}} k_{j}\right)(g k ; h k) \chi\left(g h^{-1}\right) \varphi(h) .
$$

Now if one replaces the operator $H$ with coefficients $c_{i j}, c_{i}, c_{i}^{\prime}$ and $c_{0}$ by the operator $H^{k}$ with coefficients $R_{k} c_{i j}$ etcetera, then the kernel $k_{j}$ has to be replaced by $(g, h) \mapsto$ $k_{j}(g k ; h k)$. But $H^{k} \in \mathscr{E}^{\text {div }}(\nu, 0, \nu, 0, \mu, M)$ if $H \in \mathscr{E}^{\text {div }}(\nu, 0, \nu, 0, \mu, M)$ for all $k \in G$. Therefore

$$
\rho\left(\left\{g \in G:\left|\left(R_{k} P_{j} R_{k^{-1}} \varphi\right)(g)\right|>\gamma\right\}\right) \leq M_{0} \gamma^{-1}\|\varphi\|_{\hat{i}}
$$

uniformly for all $H \in \mathscr{E}^{\text {div }}(\nu, 0, v, 0, \mu, M), k \in G, j \in \mathbb{N}$ with $j \geq 2 v, \gamma>0$ and $\varphi \in L_{1}\left(B_{1}^{\prime}\right) \cap L_{2}\left(B_{1}^{\prime}\right)$. So with (35) one establishes that

$$
\rho\left(\left\{g \in G:\left|\left(P_{j} \varphi\right)(g)\right|>\gamma\right\}\right) \leq \sum_{i=1}^{\infty} M_{0} N_{0} \gamma^{-1}\left\|R_{g_{i}}\left(\psi_{i} \varphi\right)\right\|_{\hat{\imath}}=M_{0} N_{0} \gamma^{-1}\|\varphi\|_{\hat{\imath}} .
$$

The remaining part of the proof is precisely as in [BER]. It follows that the operators $A_{i_{0}} X_{j}$ satisfy a uniform weak- $L_{\hat{1}}$ estimate and by interpolation the theorem is valid for all $p \in\langle 1,2]$.

Next assume $p \in[2, \infty)$. Now it suffices to show that the operators $\left(A_{i_{0}} X_{j}\right)^{\hat{*}}$ satisfy a uniform weak- $L_{\hat{\imath}}$ estimate since this implies that the $\left(A_{i_{0}} X_{j}\right)^{*}$ are uniformly bounded on $L_{\hat{q}}$ for all $q \in\langle 1,2]$ and then the $A_{i_{0}} X_{j}$ are uniformly bounded on $L_{\hat{p}}$. Here $\hat{*}$ denotes the dual operator with respect to the right Haar measure. But the operator $\left(A_{i_{0}} X_{j}\right)^{\hat{*}}$ has the kernel

$$
(g, h) \mapsto \overline{\left(A_{i_{0}} k_{j}\right)(h ; g)}=\int_{0}^{\infty} d t f_{j}(t) \overline{\left(A_{i_{0}} K_{t}\right)(h ; g)}
$$


Since $\left(A_{i_{0}} K_{t}\right)(h ; g)$ is Hölder continuous in $h$, with the right kind of Gaussian bounds, by Theorem 3.6, one can repeat the above arguments to deduce the validity of the theorem if $p \in[2, \infty)$.

COROLlaRY 4.7. Adopt the hypotheses of Theorem 4.6. If $c_{i j}, c_{i} \in C^{\nu '}$ for some $v \in\langle 0,1\rangle$ and $c_{i}^{\prime}, c_{0} \in L_{\infty}$ then $L_{p: 1}^{\prime} \subseteq D\left((\lambda I+H)^{1 / 2}\right)$ for all large $\lambda$ and each $p \in\langle 1, \infty\rangle$. Moreover, for all $M, \mu>0$ there exists a $\lambda_{0}>0$ such that for all $p \in\langle 1, \infty\rangle$ and $\lambda \geq \lambda_{0}$ there exists an $a>0$ such that

$$
\left\|(\lambda I+H)^{1 / 2} \varphi\right\|_{p} \leq a\|\varphi\|_{p ; 1}^{\prime}
$$

uniformly for all $H \in \mathscr{E}^{\mathrm{div}}(\nu, \nu, 0,0, \mu, M)$ and $\varphi \in L_{p ; 1}^{\prime}$.

Similar conclusions are valid on $L_{\hat{p}}$.

PROOF. This follows by duality as in [BER, p. 182].

COROLLARY 4.8. Adopt the hypotheses of Theorem 4.6. If $c_{i j}, c_{i}, c_{i}^{\prime} \in C^{\nu \prime}$ for some $v \in\langle 0,1\rangle$ and $c_{0} \in L_{\infty}$ then $L_{p ; 1}^{\prime}=D\left((\lambda I+H)^{1 / 2}\right)$ for all large $\lambda>0$ and for each $p \in\langle 1, \infty\rangle$. More generally, $L_{p ; \gamma}^{\prime}=D\left((\lambda I+H)^{\gamma / 2}\right)$ for all $\gamma \in[-1,1]$ and all large $\lambda>0$. Hence $(\lambda I+H)^{-\alpha / 2} L_{p ; \gamma}^{\prime} \subseteq L_{p ; \alpha+\gamma}^{\prime}$ for all $\gamma \in[-1,1], \alpha \in[-1-\gamma, 1-\gamma]$ and all large $\lambda>0$.

Similar conclusions are valid on the $L_{\hat{p}}$-spaces.

PROOF. The first statement follows from combination of Theorem 4.6 and Corollary 4.7. The second statement follows by the complex interpolation argument discussed prior to Theorem 4.4. The third statement is a straightforward consequence.

By definition of the quadratic form operator one immediately concludes that the operators $A_{i}(\lambda I+H)^{-1} A_{j}$ are bounded on $L_{2}$, even if all the coefficients are merely measurable. Now we prove bounds on $A_{i}(\lambda I+H)^{-1} A_{j}$ on $L_{p}$ for Hölder continuous principal coefficients. In Taylor [Tay, Theorem 2.2.H], it was established, for pure second-order strongly elliptic operators in divergence form on $\mathbb{R}^{d}$, that

$$
\left\|A_{i}(\lambda I+H)^{-1} A_{j} \varphi\right\|_{p} \leq c \sum_{k=1}^{d}\left\|(\lambda I+H)^{-1} A_{k} \varphi\right\|_{L_{q, \sigma}}+c\|\varphi\|_{p}
$$

if $1<q<p<\infty, c_{i j} \in C^{\nu}\left(\mathbb{R}^{d}\right)$ and $\sigma>1-\nu$, using the machinery of pseudodifferential operators. The next theorem improves these bounds.

THEOREM 4.9. Let $H$ be a subelliptic elliptic operator in divergence form (1). Suppose either 
(a) $H$ is strongly elliptic, or

(b) $G$ is stratified and $a_{1}, \ldots, a_{d^{\prime}}$ is a basis for $\mathfrak{g}_{1}$ in the stratification $\left(\mathfrak{g}_{m}\right)_{m \in\{1, \ldots, r\}}$ of $\mathfrak{g}$.

If $c_{i j} \in C^{\nu \prime}$, where $v \in\langle 0,1\rangle$, and $c_{i}, c_{i}^{\prime}, c_{0} \in L_{\infty}$ then $(\lambda I+H)^{-1} L_{p ;-1}^{\prime} \subseteq L_{p ; 1}^{\prime}$ for all $p \in(1, \infty)$ and large $\lambda$.

Moreover, for all $M>0$ and $p \in\langle 1, \infty\rangle$ there exist $\lambda_{0}, a>0$ such that

$$
\begin{gathered}
\left\|(\lambda I+H)^{-1}\right\|_{L_{p,-1}^{\prime} \rightarrow L_{p: 1}^{\prime}} \leq a, \\
\left\|(\lambda I+H)^{-1}\right\|_{L_{p:-1}^{\prime} \rightarrow L_{p}} \leq a \lambda^{-1 / 2} \quad \text { and }\left\|(\lambda I+H)^{-1}\right\|_{L_{p} \rightarrow L_{p: 1}^{\prime}} \leq a \lambda^{-1 / 2}
\end{gathered}
$$

uniformly for all $\lambda \geq \lambda_{0}$ and $H \in \mathscr{E}^{\mathrm{div}}(\nu, 0,0,0, \mu, M)$.

Similar statements are valid on the $L_{\hat{p}}$-spaces.

PROOF. Let $H_{0}=-\sum_{i, j=1}^{d^{\prime}} A_{i} c_{i j} A_{j}+c_{0} I$. It follows from Theorem 4.6 and duality that the operators $A_{i}\left(\lambda I+H_{0}\right)^{-1 / 2}$ and $A_{j}\left(\lambda I+H_{0}^{*}\right)^{-1 / 2}$ are bounded on $L_{p}$ and $L_{p^{*}}$, respectively, uniformly for all large $\lambda$, where $1 / p+1 / p^{*}=1$ and $p \in(1, \infty)$. Therefore there exists a $\lambda_{0}>0$ such that for all $p \in\langle 1, \infty\rangle$ there exists an $a_{p}>0$ such that

$$
\left\|A_{i}\left(\lambda I+H_{0}\right)^{-1} A_{j}\right\|_{p \rightarrow p}=\left\|A_{i}\left(\lambda I+H_{0}\right)^{-1 / 2}\left(A_{j}\left(\lambda I+H_{0}^{*}\right)^{-1 / 2}\right)^{*}\right\|_{p \rightarrow p} \leq a_{p}
$$

uniformly for all $\lambda \geq \lambda_{0}$. Moreover, Corollaries 3.8 and 3.9 give the bounds $\| A_{i}(\lambda I+$ $\left.H_{0}\right)^{-1} \|_{p \rightarrow p} \leq a \lambda^{-1 / 2}$ and $\left\|\left(\lambda I+H_{0}\right)^{-1} A_{j}\right\|_{p \rightarrow p} \leq a \lambda^{-1 / 2}$ for a suitable $a>0$, uniformly for all $\lambda \geq \lambda_{0}$ and $p \in[1, \infty]$, possibly by increasing $\lambda_{0}$.

Next let $V=\sum_{k=1}^{d^{\prime}} c_{i} A_{i}$. Then

$$
\left\|V\left(\lambda I+H_{0}\right)^{-1}\right\|_{p \rightarrow p} \leq \sum_{k=1}^{d^{\prime}}\left\|c_{k}\right\|_{\infty}\left\|A_{k}\left(\lambda I+H_{0}\right)^{-1}\right\|_{p \rightarrow p} \leq a^{\prime} \lambda^{-1 / 2}
$$

and similarly $\left\|V\left(\lambda I+H_{0}\right)^{-1} A_{j}\right\|_{p \rightarrow p} \leq a_{p}^{\prime}$ uniformly for all $\lambda \geq \lambda_{0}$. The value of $a^{\prime}$ is independent of $p$. Therefore, if $\lambda \geq \lambda_{0} \vee\left(2 a^{\prime}\right)^{2}$ the series

$$
R^{(p)}=\left(\lambda I+H_{0}\right)^{-1} \sum_{n=0}^{\infty}\left(-V\left(\lambda I+H_{0}\right)^{-1}\right)^{n}
$$

is norm convergent on $L_{\rho}$. But a standard perturbation argument establishes that $R_{\lambda}^{(p)}=\left(\lambda I+H^{(p)}\right)^{-1}$ where $H^{(p)}$ is the $L_{p}$-closure of the operator sum $H_{0}+V$. Moreover, by comparison of convergent series $R_{\lambda}^{(p)} \varphi=R_{\lambda}^{(q)} \varphi$ for all $\varphi \in L_{p} \cap L_{q}$, each pair $p, q \in\langle 1, \infty\rangle$ and all appropriately large $\lambda$. Now we must argue that the $H^{(p)}$ are the $L_{p}$-versions of $H_{1}$, the sectorial operator with coefficients $c_{i j}, c_{i}, c_{0}$ defined on $L_{2}$ by the form (3). Thus we must establish that $\left(\lambda I+H_{1}\right)^{-1} \varphi=R_{\lambda}^{(2)} \varphi$ for 
all $\varphi \in L_{2}$. If $h_{1}$ and $h_{0}$ are the sesquilinear forms defining the operators $H$ and $H_{0}$, respectively, then it suffices to prove that

$$
\lambda\left(\psi, R_{\lambda}^{(2)} \varphi\right)+h_{1}\left(\psi, R_{\lambda}^{(2)} \varphi\right)=(\psi, \varphi)
$$

for all $\psi \in L_{2 ; 1}^{\prime}$ and $\varphi \in L_{2}$. But $R_{\lambda}^{(2)} \varphi \in\left(\lambda I+H_{0}\right)^{-1} L_{2} \subset D\left(H_{0}\right) \subset L_{2 ; 1}^{\prime}$ and

$$
R_{\lambda}^{(2)} \varphi=\sum_{n=0}^{\infty}\left(\lambda I+H_{0}\right)^{-1}\left(-V\left(\lambda I+H_{0}\right)^{-1}\right)^{n} \varphi
$$

in $L_{2 ; 1}^{\prime}$. So

$$
\begin{aligned}
\lambda\left(\psi, R_{\lambda}^{(2)} \varphi\right)+h_{1}\left(\psi, R_{\lambda}^{(2)} \varphi\right) & \\
= & \lambda\left(\psi, R_{\lambda}^{(2)} \varphi\right)+\sum_{n=0}^{\infty} h_{0}\left(\psi,\left(\lambda I+H_{0}\right)^{-1}\left(-V\left(\lambda I+H_{0}\right)^{-1}\right)^{n} \varphi\right)+\left(\psi, V R_{\lambda}^{(2)} \varphi\right) \\
= & \lambda \sum_{n=0}^{\infty}\left(\psi,\left(\lambda I+H_{0}\right)^{-1}\left(-V\left(\lambda I+H_{0}\right)^{-1}\right)^{n} \varphi\right) \\
& +\sum_{n=0}^{\infty}\left(\psi, H_{0}\left(\lambda I+H_{0}\right)^{-1}\left(-V\left(\lambda I+H_{0}\right)^{-1}\right)^{n} \varphi\right) \\
& +\sum_{n=0}^{\infty}\left(\psi, V\left(\lambda I+H_{0}\right)^{-1}\left(-V\left(\lambda I+H_{0}\right)^{-1}\right)^{n} \varphi\right)=(\psi, \varphi) .
\end{aligned}
$$

Therefore $\left(\lambda I+H_{1}\right)^{-1}=R^{(2)}$.

Next it follows that

$$
A_{i}\left(\lambda I+H_{1}\right)^{-1}=A_{i}\left(\lambda I+H_{0}\right)^{-1} \sum_{n=0}^{\infty}\left(-V\left(\lambda I+H_{0}\right)^{-1}\right)^{n}
$$

and

$$
\begin{aligned}
\left\|A_{i}\left(\lambda I+H_{1}\right)^{-1}\right\|_{p \rightarrow p} & \leq\left\|A_{i}\left(\lambda I+H_{0}\right)^{-1}\right\|_{p \rightarrow p} \sum_{n=0}^{\infty}\left(a^{\prime} \lambda^{-1 / 2}\right)^{n} \\
& \leq a \lambda^{-1 / 2}\left(1-a^{\prime} \lambda^{-1 / 2}\right)^{-1} \leq 2 a \lambda^{-1 / 2}
\end{aligned}
$$

Alternatively,

$$
\begin{aligned}
A_{i}(\lambda I & \left.+H_{1}\right)^{-1} A_{j} \\
& =A_{i}\left(\lambda I+H_{0}\right)^{-1} A_{j}-A_{i}\left(\lambda I+H_{0}\right)^{-1} \sum_{n=1}^{\infty}\left(-V\left(\lambda I+H_{0}\right)^{-1}\right)^{n-1} V\left(\lambda I+H_{0}\right)^{-1} A_{j}
\end{aligned}
$$


and

$$
\begin{aligned}
\left\|A_{i}\left(\lambda I+H_{1}\right)^{-1} A_{j}\right\|_{p \rightarrow p} & \leq\left\|A_{i}\left(\lambda I+H_{0}\right)^{-1} A_{j}\right\|_{p \rightarrow p}+\sum_{n=1}^{\infty} a \lambda^{-1 / 2}\left(a^{\prime} \lambda^{-1 / 2}\right)^{n-1} a_{p}^{\prime} \\
& \leq\left\|A_{i}\left(\lambda I+H_{0}\right)^{-1} A_{j}\right\|_{p \rightarrow p}+a a_{p}^{\prime} \lambda^{-1 / 2}\left(1-a^{\prime} \lambda^{-1 / 2}\right)^{-1} \leq a_{p}^{\prime \prime}
\end{aligned}
$$

for some $a_{p}^{\prime \prime}>0$, independent of $\lambda$ whenever $\lambda \geq \lambda_{0} \vee\left(2 a^{\prime}\right)^{2}$. Analogously, $\|(\lambda I+$ $\left.H_{1}\right)^{-1} A_{j} \|_{p \rightarrow p} \leq a_{p}^{\prime \prime} \lambda^{-1 / 2}$.

By duality one then obtains bounds

$$
\left\|A_{i}\left(\lambda I+H_{1}^{*}\right)^{-1}\right\|_{p \rightarrow p} \leq a_{p}^{\prime \prime} \lambda^{-1 / 2}, \quad\left\|\left(\lambda I+H_{1}^{*}\right)^{-1} A_{j}\right\|_{p \rightarrow p} \leq 2 a \lambda^{-1 / 2}
$$

and $\left\|A_{i}\left(\lambda I+H_{1}^{*}\right)^{-1} A_{j}\right\|_{p \rightarrow p} \leq a_{p}^{\prime \prime}$ uniformly for all $\lambda \geq \lambda_{0}$ and $p \in\langle 1, \infty\rangle$. Therefore by repetition of these arguments one can add the term $V^{\prime}=-\sum_{k=1}^{d^{\prime}} c_{k}^{\prime} A_{k}$ to $H_{1}^{*}$ and deduce the required bounds on the derivatives of $\left(\lambda I+H^{*}\right)^{-1}$. These bounds require that $a_{p}^{\prime \prime} \lambda^{-1 / 2} \leq 1 / 2$, so now $\lambda_{0}$ depends on $p$. Then, by duality, one obtains the bounds on $(\lambda I+H)^{-1}$. The uniformity of the constants for all $H \in \mathscr{E}^{\text {div }}(\nu, 0,0,0, \mu, M)$ follows from the proof.

\section{Operators with $L_{\infty: n}$-coefficients, $n \in \mathbb{N}$}

In this section we consider strongly elliptic operators in non-divergence form (4),

$$
H=-\sum_{i, j=1}^{d} c_{i j} A_{i} A_{j}+\sum_{i=1}^{d} c_{i} A_{i}+c_{0} I,
$$

with coefficients $c_{i j} \in L_{\infty ; 1}$ and $c_{i}, c_{0} \in L_{\infty}$ and again examine differentiability and smoothness of the corresponding kernels. Since the operators are no longer expressed in a symmetric form one would expect an imbalance between the differentiability properties of the kernel with respect to the first and second variable. For example, the kernel should be less smooth with respect to the second variable than in the earlier case of divergence form operators. What is less evident is that the kernel is even smoother with respect to the first variable. There is an improvement of regularity by two derivatives and not just one as before.

For $N_{2} \in \mathbb{N}, N_{1}, N_{0} \in \mathbb{N}_{0}, \nu_{2}, v_{1}, v_{0} \in[0,1\rangle, \mu>0$ and $M>0$ such that $N_{2}+v_{2} \geq N_{1}+v_{1} \geq N_{0}+v_{0}$ let $\mathscr{E}^{\text {nondiv }}\left(N_{2}+v_{2}, N_{1}+v_{1}, N_{0}+v_{0}, \mu, M\right)$ be the set of all second-order strongly elliptic operators of the form (4) such that $c_{i j} \in L_{\infty ; N_{2}}$, $\left\|c_{i j}\right\|_{\infty ; N_{2}} \leq M$, the ellipticity constant $\mu_{C} \geq \mu$ and if $\nu_{2}>0$ then $\left\|A^{\alpha} c_{i j}\right\|_{C^{\nu}} \leq M$ for all $\alpha \in J_{N_{2}}(d)$ and similar conditions on the $c_{i}$ and $c_{0}$. Since $c_{i j} \in L_{\infty ; 1}$ each operator 
in non-divergence form can be written as an operator in divergence form without the terms with $c_{i}^{\prime}$. Explicitly,

$$
\mathscr{E}^{\text {nondiv }}\left(N_{2}+v_{2}, N_{1}+v_{1}, N_{0}+v_{0}, \mu, M\right)
$$

(36)

$$
\subset \mathscr{E}^{\mathrm{div}}\left(N_{2}+v_{2},\left(N_{1}+v_{1}\right) \wedge\left(N_{2}+v_{2}-1\right), P, N_{0}+v_{0}, \mu,(d+1) M\right)
$$

for all $P \geq 0$. Moreover, if $N_{1}^{\prime} \in \mathbb{N}$ and $\nu_{1}^{\prime} \in[0,1\rangle$ then

$$
\begin{gathered}
\mathscr{E}^{\text {div }}\left(N_{2}+v_{2}, N_{1}+v_{1}, N_{1}^{\prime}+v_{1}^{\prime}, N_{0}+v_{0}, \mu, M\right) \\
\subset \mathscr{E}^{\text {nondiv }}\left(N_{2}+v_{2},\left(N_{1}+v_{1}\right) \wedge\left(N_{1}^{\prime}+v_{1}^{\prime}\right) \wedge\left(N_{2}+v_{2}-1\right),\right. \\
\left.\left(N_{0}+v_{0}\right) \wedge\left(N_{1}^{\prime}+v_{1}^{\prime}-1\right), \mu,(d+2) M\right) .
\end{gathered}
$$

Similarly,

$$
\left\{H^{*}: H \in \mathscr{E}^{\text {nondiv }}\left(N_{2}+v_{2}, N_{1}+v_{1}, N_{0}+v_{0}, \mu, M\right)\right\}
$$

$$
\subset \mathscr{E}^{\mathrm{div}}\left(N_{2}+v_{2}, P,\left(N_{1}+v_{1}\right) \wedge\left(N_{2}+v_{2}-1\right), N_{0}+v_{0}, \mu,(d+1) M\right)
$$

for all $P \geq 0$.

PROPOSITION 5.1. Let $H$ be a strongly elliptic operator in non-divergence form (4) with complex coefficients $c_{i j} \in L_{\infty ; 1}$ and $c_{i}, c_{0} \in L_{\infty}$. If $p \in\langle 1, \infty\rangle$ then $(\lambda I+$ $H)^{-1} L_{p} \subseteq L_{p: 2}$ for large $\lambda$.

Moreover, for all $M, \mu>0$ there exists a $\lambda_{0}>0$ such that for all $p \in\langle 1, \infty\rangle$ there exists an $a>0$ such that

$$
\left\|A^{\alpha}(\lambda I+H)^{-1}\right\|_{p \rightarrow p} \leq a \lambda^{-(2-|\alpha|) / 2}
$$

for all $\alpha$ with $|\alpha| \leq 2$ uniformly for all $\lambda \geq \lambda_{0}$ and $H \in \mathscr{E}^{\text {nondiv }}(1,0,0, \mu, M)$.

Similar statements are valid on the $L_{\hat{p}}$-spaces.

PROOF. By Corollary 3.8 the bounds (39) for $|\alpha|=1$ are valid for large $\lambda$, uniformly for all $p \in[1, \infty]$.

For the case $|\alpha|=2$ it suffices to prove the bounds (38) for one particular $\lambda$, dependent on $p$, by the argument at the beginning of the proof of Theorem 4.4. So fix $p \in\langle 1, \infty\rangle$. Let $H_{2}=-\sum_{i, j=1}^{d} A_{i} c_{i j} A_{j}$. Then $H=H_{2}+H_{1}$, where $H_{1}=\sum_{i, j=1}^{d}\left(\left(A_{i} c_{i j}\right)+c_{j}\right) A_{j}+c_{0} I$. 
Next let $i_{0}, j_{0} \in\{1, \ldots, d\}$. Then the operators $A_{i_{0}}\left(\lambda I+H_{2}\right)^{-1} A_{j_{0}}$ are uniformly bounded for all large $\lambda$ by an application of Theorem 4.9. Moreover,

$$
\begin{aligned}
A_{j_{0}}\left(\lambda I+H_{2}\right)^{-1}= & \left(\lambda I+H_{2}\right)^{-1} A_{j_{0}}-\left(\lambda I+H_{2}\right)^{-1}\left[A_{j_{0}}, H_{2}\right]\left(\lambda I+H_{2}\right)^{-1} \\
= & \left(\lambda I+H_{2}\right)^{-1} A_{j_{0}}+\sum_{i, j, k=1}^{d} c_{j_{0} i}^{k}\left(\lambda I+H_{2}\right)^{-1} A_{k} c_{i j} A_{j}\left(\lambda I+H_{2}\right)^{-1} \\
& +\sum_{i, j=1}^{d}\left(\lambda I+H_{2}\right)^{-1} A_{i}\left(A_{j_{0}} c_{i j}\right) A_{j}\left(\lambda I+H_{2}\right)^{-1} \\
& +\sum_{i, j, k=1}^{d} c_{j_{0} j}^{k}\left(\lambda I+H_{2}\right)^{-1} A_{i} c_{i j} A_{k}\left(\lambda I+H_{2}\right)^{-1}
\end{aligned}
$$

where $c_{i j}^{k}$ are the structure constants of the Lie algebra with respect to the basis $a_{1}, \ldots, a_{d}$. Therefore

$$
\begin{aligned}
A_{i_{0}} A_{j_{0}}\left(\lambda I+H_{2}\right)^{-1}= & A_{i_{0}}\left(\lambda I+H_{2}\right)^{-1} A_{j_{0}} \\
& +\sum_{i, j, k=1}^{d} c_{j_{0} i}^{k}\left(A_{i_{0}}\left(\lambda I+H_{2}\right)^{-1} A_{k}\right) c_{i j}\left(A_{j}\left(\lambda I+H_{2}\right)^{-1}\right) \\
& +\sum_{i, j=1}^{d}\left(A_{i_{0}}\left(\lambda I+H_{2}\right)^{-1} A_{i}\right)\left(A_{j_{0}} c_{i j}\right)\left(A_{j}\left(\lambda I+H_{2}\right)^{-1}\right) \\
& +\sum_{i, j, k=1}^{d} c_{j_{0} j}^{k}\left(A_{i_{0}}\left(\lambda I+H_{2}\right)^{-1} A_{i}\right) c_{i j}\left(A_{k}\left(\lambda I+H_{2}\right)^{-1}\right) .
\end{aligned}
$$

It now follows easily from Theorem 4.9 that the operators $A_{i_{0}} A_{j_{0}}\left(\lambda I+H_{2}\right)^{-1}$ are uniformly bounded on $L_{p}$ for all large $\lambda$. Again by Corollary 3.8 one has

$$
\left\|H_{1}\left(\lambda I+H_{2}\right)^{-1}\right\|_{p \rightarrow p} \leq a \lambda^{-1 / 2}
$$

for some $a>0$ uniformly for all large $\lambda$ and $p \in[1, \infty]$. Then the perturbation series

$$
(\lambda I+H)^{-1}=\sum_{n=0}^{\infty}\left(\lambda I+H_{2}\right)^{-1}\left(-H_{1}\left(\lambda I+H_{2}\right)^{-1}\right)^{n}
$$

converges and

$$
A_{i_{0}} A_{j_{0}}(\lambda I+H)^{-1}=\sum_{n=0}^{\infty}\left(A_{i_{0}} A_{j_{0}}\left(\lambda I+H_{2}\right)^{-1}\right)\left(-H_{1}\left(\lambda I+H_{2}\right)^{-1}\right)^{n}
$$

is a bounded operator on $L_{p}$. 
COROLLARY 5.2. Let $H$ be a strongly elliptic operator in non-divergence form (4) with complex coefficients $c_{i j}, c_{i}, c_{0} \in L_{\infty: n}$, where $n \in \mathbb{N}$. If $p \in(1, \infty)$ then $D\left((\lambda I+H)^{(n+2) / 2}\right)=L_{p ; n+2}$ for all large $\lambda$, with equivalent norms.

Moreover, for all $M, \mu>0$ there exists a $\lambda_{0}>0$ such that for all $p \in\langle 1, \infty\rangle$ there exists an $a>0$ such that

$$
\left\|A^{\alpha}(\lambda I+H)^{-(n+2) / 2}\right\|_{p \rightarrow p} \leq a \lambda^{-(n+2-|\alpha|) / 2}
$$

uniformly for all $H \in \mathscr{E}^{\text {nondiv }}(n, n, n, \mu, M), \lambda \geq \lambda_{0}$ and $\alpha \in J_{n+2}(d)$. Also, for all $\lambda \geq \lambda_{0}$ and $p \in\langle 1, \infty\rangle$ there exists an $a>0$ such that

$$
\left\|(\lambda I+H)^{(n+2) / 2} \varphi\right\|_{p} \leq a\|\varphi\|_{p ; n+2}
$$

uniformly for all $\varphi \in L_{p: n+2}$ and $H \in \mathscr{E}^{\text {nondiv }}(n, n, n, \mu, M)$.

Similar statements are valid on the $L_{\hat{p}}$-spaces.

PROOF. For each $n \in \mathbb{N}_{0}$ introduce the hypothesis $P(n)$ by

for all $M, \mu>0$ and $p \in\langle 1, \infty\rangle$ there exist $\lambda_{0}>0$ and $a>0$ such that

$$
\begin{aligned}
& (\lambda I+H)^{-(n+2) / 2} L_{p} \subseteq L_{p ; n+2} \text { and } \\
& \quad\left\|A^{\alpha}(\lambda I+H)^{-(n+2) / 2}\right\|_{p \rightarrow p} \leq a \lambda^{-(n+2-|\alpha|) / 2}
\end{aligned}
$$

uniformly for all $\lambda \geq \lambda_{0}, \alpha \in J_{n+2}(d)$ and $H \in \mathscr{E}^{\text {enondiv }}(n \vee 1, n, n, \mu, M)$.

Then Proposition 5.1 states that $P(0)$ is valid. If we can prove that $P(n)$ is valid for all $n$ then one deduces again from the holomorphic functional calculus that the first part of the corollary is valid uniformly for all large $\lambda$, independent of $p$.

Let $n \in \mathbb{N}$ and suppose that $P(n-1)$ is valid. We have to distinguish between even and odd $n$.

CASE 1. Suppose $n=2 m-1$ with $m \in \mathbb{N}$.

Let $H$ be a strongly elliptic operator in non-divergence form with $n$-times differentiable coefficients. Fix $i \in\{1, \ldots, d\}$ then

$$
\left[(\lambda I+H)^{m}, A_{i}\right]=\sum_{k=1}^{m}\left(\begin{array}{c}
m \\
k
\end{array}\right)(\operatorname{ad} H)^{k}\left(A_{i}\right)(\lambda I+H)^{m-k}
$$

and

$$
(\operatorname{ad} H)^{k}\left(A_{i}\right)=\sum_{\alpha \in J_{k+1}(d)} g_{k, \alpha} A^{\alpha}
$$

with $g_{k, \alpha} \in L_{\infty}$. Therefore 


$$
\begin{aligned}
\left\|\left[A_{i},(\lambda I+H)^{m}\right](\lambda I+H)^{-m}\right\|_{p \rightarrow p} & \leq \sum_{k=1}^{m}\left(\begin{array}{c}
m \\
k
\end{array}\right) \sum_{\alpha \in J_{k+1}(d)}\left\|g_{k, \alpha}\right\|_{\infty}\left\|A^{\alpha}(\lambda I+H)^{-k}\right\|_{p \rightarrow p} \\
& \leq a \sum_{k=1}^{m}\left(\begin{array}{c}
m \\
k
\end{array}\right) \sum_{\alpha \in J_{k+1}(d)}\left\|g_{k, \alpha}\right\|_{\infty} \lambda^{-(k-|\alpha| / 2)} \leq a^{\prime}
\end{aligned}
$$

by the induction hypothesis $P(n-1)$, uniformly for all large $\lambda$. Hence

$$
\begin{aligned}
A_{i}(\lambda I+H)^{-(n+2) / 2}= & A_{i}(\lambda I+H)^{-m}(\lambda I+H)^{-1 / 2} \\
= & (\lambda I+H)^{-m} A_{i}(\lambda I+H)^{-1 / 2} \\
& +(\lambda I+H)^{-m}\left(\left[(\lambda I+H)^{m}, A_{i}\right](\lambda I+H)^{-m}\right)(\lambda I+H)^{-1 / 2} .
\end{aligned}
$$

So for all $\alpha \in J_{n+1}(d)=J_{2 m}(d)$ one has

$$
\begin{aligned}
& \left\|A^{\alpha} A_{i}(\lambda I+H)^{-(n+2) / 2}\right\|_{p \rightarrow p} \\
& \quad \leq\left\|\left(A^{\alpha}(\lambda I+H)^{-m}\right)\left(A_{i}(\lambda I+H)^{-1 / 2}\right)\right\|_{p \rightarrow p} \\
& \quad+\left\|\left(A^{\alpha}(\lambda I+H)^{-m}\right)\left(\left[A_{i},(\lambda I+H)^{m}\right](\lambda I+H)^{-m}\right)(\lambda I+H)^{-1 / 2}\right\|_{p \rightarrow p} \\
& \leq a \lambda^{-(m-|\alpha| / 2)}+a^{\prime} \lambda^{-(m-|\alpha| / 2)} \lambda^{-1 / 2} \leq a^{\prime \prime} \lambda^{-(n+2-(|\alpha|+1)) / 2}
\end{aligned}
$$

uniformly for all large $\lambda$, by the induction hypothesis $P(n-1)$. Therefore $P(n)$ is valid if $n$ is odd, since one always has the bounds $\left\|(\lambda I+H)^{-(n+2) / 2}\right\|_{p \rightarrow p} \leq a \lambda^{-(n+2) / 2}$ for large $\lambda$.

CASE 2. Suppose $n=2 m$ with $m \in \mathbb{N}$.

Let $H$ be a strongly elliptic operator in non-divergence form with $n$-times differentiable coefficients. Now one has

$$
\left[(\lambda I+H)^{m}, A_{i} A_{j}\right]=\sum_{k=1}^{m}\left(\begin{array}{c}
m \\
k
\end{array}\right)(\operatorname{ad} H)^{k}\left(A_{i} A_{j}\right)(\lambda I+H)^{m-k}
$$

and

$$
(\operatorname{ad} H)^{k}\left(A_{i} A_{j}\right)=\sum_{\alpha \in J_{k+2}(d)} g_{k . \alpha} A^{\alpha}
$$

where $g_{k, \alpha} \in L_{\infty}$. One concludes, again, that $\left\|\left[A_{i} A_{j},(\lambda I+H)^{m}\right](\lambda I+H)^{-(2 m+1) / 2}\right\|_{p \rightarrow p}$ is bounded uniformly for large $\lambda$ by the induction hypothesis. On the other hand,

$$
\begin{aligned}
& A_{i} A_{j}(\lambda I+H)^{-(n+2) / 2}=(\lambda I+H)^{-m} A_{i} A_{j}(\lambda I+H)^{-1} \\
& \quad+(\lambda I+H)^{-m}\left(\left[(\lambda I+H)^{m}, A_{i} A_{j}\right](\lambda I+H)^{-(2 m+1) / 2}\right)(\lambda I+H)^{-1 / 2}
\end{aligned}
$$


and, arguing as above, one deduces that $P(n)$ is valid for $n$ even.

Finally we show that $L_{p ; n+2} \subseteq D\left((\lambda I+H)^{(n+2) / 2}\right)$ for each $\lambda \geq \lambda_{0}$. Fix $\lambda \geq \lambda_{0}$. Suppose $n=2 m$ is even, with $m \in \mathbb{N}$. Then

$$
(\lambda I+H)^{(n+2) / 2}=(\lambda I+H)^{m+1}=\sum_{\alpha \in J_{n+2}(d)} f_{\alpha} A^{\alpha}
$$

with $f_{\alpha} \in L_{\infty}$, and $\left\|f_{\alpha}\right\|_{\infty}$ depends only on $M, \lambda$ and $m$. Then

$$
\left\|(\lambda I+H)^{(n+2) / 2} \varphi\right\|_{p} \leq \sum_{\alpha \in J_{n+2}(d)}\left\|f_{\alpha}\right\|_{\infty}\|\varphi\|_{p ; n+2} .
$$

The case $n=2 m+1$, with $m \in \mathbb{N}_{0}$ is slightly more complicated. By Corollary 4.7 there exists an $a>0$, depending only on $\mu, M$ and $p$, such that $\left\|(\lambda I+H)^{1 / 2} \varphi\right\|_{p} \leq$ $a\|\varphi\|_{p ; 1}$ for all $\varphi \in L_{p ; 1}$. Now

$$
(\lambda I+H)^{(n+1) / 2}=(\lambda I+H)^{m+1}=\sum_{\alpha \in J_{n+1}(d)} f_{\alpha} A^{\alpha}
$$

with $f_{\alpha} \in L_{\infty ; 1}$, and $\left\|f_{\alpha}\right\|_{\infty ; 1}$ depends only on $M, \lambda$ and $m$. Hence one has

$$
\begin{aligned}
\left\|(\lambda I+H)^{(n+2) / 2} \varphi\right\|_{p} & =\left\|(\lambda I+H)^{1 / 2}(\lambda I+H)^{m+1} \varphi\right\|_{p} \leq a\left\|(\lambda I+H)^{m+1} \varphi\right\|_{p ; 1} \\
& \leq a \sum_{\alpha \in J_{n+1}(d)}\left\|f_{\alpha} A^{\alpha} \varphi\right\|_{p ; 1} \leq a_{\lambda}\|\varphi\|_{p: n+2}
\end{aligned}
$$

for all $\varphi \in L_{p: n+2}$ and an appropriate $a_{\lambda}>0$.

Next we use these bounds to improve heat kernel bounds for semigroups generated by non-divergence form operators with differentiable coefficients. We need several lemmas.

LEMMA 5.3. For all $n \in\{-1,0,1,2, \ldots\}, p \in\langle 1, \infty)$ and $M, \mu>0$ there exist $a, \omega>0$ such that $\left\|A^{\alpha} S_{t}^{\rho}\right\|_{\hat{p} \rightarrow \hat{p}} \leq a^{-|\alpha| / 2} e^{\omega\left(1+\rho^{2}\right) t}$ uniformly for all $H \in \mathscr{E}^{\text {nondiv }}(n \vee$ $1, n \vee 0, n \vee 0, \mu, M), \alpha \in J_{n+2}(d), t>0, \rho \in \mathbb{R}$ and perturbation functions $\psi \in C_{c}^{\infty}(G)$ with $\left\|A^{\beta} \psi\right\|_{\infty} \leq 1$ for all $\beta \in J(d)$ with $1 \leq|\beta| \leq n+2$.

PROOF. The proof is by induction on $n$. The case $n=-1$ has been proved in Corollary 3.7.

Let $n \in \mathbb{N}_{0}, H \in \mathscr{E}^{\text {nondiv }}(n \vee 1, n, n, \mu, M)$ and $\alpha \in J_{n+2}(d)$. We may assume that $|\alpha|=n+2$. Set $H_{2}=-\sum_{i, j=1}^{d} c_{i j} A_{i} A_{j}$. By Corollary 5.2 there exist $a, \lambda_{0}>0$, depending only on $M, \mu$ and $p$, such that

$$
\|\eta\|_{\hat{p} ; n+2} \leq a\left\|\left(\lambda_{0} I+H_{2}\right)^{(n+2) / 2} \eta\right\|_{\hat{p}}, \quad\left\|\left(\lambda_{0} I+H_{2}\right)^{n / 2} \eta\right\|_{\hat{p}} \leq a\|\eta\|_{\hat{p} ; n}
$$


uniformly for all $\eta \in L_{\hat{p} ; n+2}$. Then for all $\varphi \in L_{\hat{p} ; n+2}$ one has $\left\|A^{\alpha} S_{t}^{\rho} \varphi\right\|_{\hat{p}} \leq\left\|S_{t}^{\rho} \varphi\right\|_{\hat{p} ; n+2} \leq a\left\|\left(\lambda_{0} I+H_{2}\right)^{n / 2}\left(\lambda_{0} I+H_{2}\right) S_{t}^{\rho} \varphi\right\|_{\hat{p}} \leq a^{2}\left\|\left(\lambda_{0} I+H_{2}\right) S_{t}^{\rho} \varphi\right\|_{\hat{p} ; n}$. Now

$$
\begin{aligned}
H_{2} & =H_{\rho}+\rho \sum_{i, j=1}^{d} c_{i j} A_{i} \psi_{j}+\rho \sum_{i, j=1}^{d} c_{i j} \psi_{i} A_{j}+\rho^{2} \sum_{i, j=1}^{d} c_{i j} \psi_{i} \psi_{j}-\sum_{i=1}^{d} c_{i} A_{i}-\rho \sum_{i=1}^{d} c_{i} \psi_{i}-c_{0} I \\
& =H_{\rho}+\rho^{2} V_{1}+\rho V_{2}+V_{3},
\end{aligned}
$$

where $V_{1}, V_{2}, V_{3}$ are differential operators in non-divergence form of order 0,1 and 1 with $L_{\infty ; n}$ coefficients, whose $L_{\infty ; n}$-norm depend only on $M$ and the $\left\|A^{\beta} \psi\right\|_{\infty}$ with $1 \leq|\beta| \leq 2$. So

$$
\begin{gathered}
\left\|A^{\alpha} S_{t}^{\rho} \varphi\right\|_{\hat{p}} \leq a^{2} \lambda_{0}\left\|S_{t}^{\rho} \varphi\right\|_{\hat{p} ; n}+a^{2}\left\|H_{\rho} S_{t}^{\rho} \varphi\right\|_{\hat{p} ; n}+a^{2} \rho^{2}\left\|V_{1} S_{t}^{\rho} \varphi\right\|_{\hat{p} ; n} \\
+a^{2}|\rho|\left\|V_{2} S_{t}^{\rho} \varphi\right\|_{\hat{p} ; n}+a^{2}\left\|V_{3} S_{t}^{\rho} \varphi\right\|_{\hat{p} ; n} .
\end{gathered}
$$

We estimate the five contributions separately. First, by the induction hypothesis

$$
\left\|S_{t}^{\rho} \varphi\right\|_{\hat{p} ; n} \leq a^{\prime} t^{-n / 2} e^{\omega\left(1+\rho^{2}\right) t}\|\varphi\|_{\hat{p}} \leq a^{\prime \prime} t^{-(n+2) / 2} e^{\omega^{\prime}\left(1+\rho^{2}\right) t}\|\varphi\|_{\hat{p}} .
$$

Secondly, it follows from Corollary 3.7 and [Rob, Lemma III.4.4], that

$$
\left\|H_{\rho} S_{t}^{\rho}\right\|_{\hat{p} \rightarrow \hat{p}} \leq a^{\prime} t^{-1} e^{\omega\left(1+\rho^{2}\right) t}
$$

for suitable $a^{\prime}, \omega>0$. Hence, by the induction hypothesis,

$$
\begin{aligned}
\left\|H_{\rho} S_{t}^{\rho} \varphi\right\|_{\hat{p} ; n} & =\left\|S_{t / 2}^{\rho} H_{\rho} S_{t / 2}^{\rho} \varphi\right\|_{\hat{p} ; n} \leq\left\|S_{t / 2}^{\rho}\right\|_{L_{\hat{p}} \rightarrow L_{\hat{p} ; n}}\left\|H_{\rho} S_{t / 2}^{\rho}\right\|_{\hat{p} \rightarrow \hat{p}}\|\varphi\|_{\hat{p}} \\
& \leq a^{\prime \prime} t^{-(n+2) / 2} e^{\omega^{\prime}\left(1+\rho^{2}\right) t}\|\varphi\|_{\hat{p}} .
\end{aligned}
$$

Thirdly, $\rho^{2}\left\|V_{\mathrm{I}} S_{t}^{\rho} \varphi\right\|_{\hat{p} ; n}=\rho^{2} \max _{\beta \in J_{n}(d)}\left\|A^{\beta} V_{\mathrm{I}} S_{t}^{\rho} \varphi\right\|_{\hat{p}}$. But $A^{\beta} V_{\mathrm{I}}$ is a differential operator in non-divergence form of order $|\beta| \leq n$ with $L_{\infty}$-coefficients, whose $L_{\infty}$ norm depends on $M$ and the $\left\|A^{\gamma} \psi\right\|_{\infty}$ with $1 \leq|\gamma| \leq n+2$. So

$$
\rho^{2}\left\|V_{1} S_{t}^{\rho} \varphi\right\|_{\hat{p} ; n} \leq \rho^{2} a^{\prime} t^{-n / 2} e^{\omega\left(1+\rho^{2}\right) t}\|\varphi\|_{\hat{p}} \leq a^{\prime \prime} t^{-(n+2) / 2} e^{\omega^{\prime}\left(1+\rho^{2}\right) t}\|\varphi\|_{\hat{p}} .
$$

Fourthly, for all $\beta \in J_{n}(d)$ the operator $A^{\beta} V_{2}$ is a differential operator in nondivergence form of order $|\beta|+1 \leq n+1$ with $L_{\infty}$-coefficients, whose $L_{\infty}$-norm depends on $M$ and the $\left\|A^{\gamma} \psi\right\|_{\infty}$ with $1 \leq|\gamma| \leq n+2$. So

$$
|\rho|\left\|V_{2} S_{t}^{\rho} \varphi\right\|_{\hat{p} ; n} \leq\left(1+\rho^{2}\right)^{1 / 2} a^{\prime} t^{-(n+1) / 2} e^{\omega\left(1+\rho^{2}\right) t}\|\varphi\|_{\hat{p}} \leq a^{\prime \prime} t^{-(n+2) / 2} e^{\omega^{\prime}\left(1+\rho^{2}\right) t}\|\varphi\|_{\hat{p}} .
$$

The fifth term can be estimated similarly to the fourth. Adding the five contributions one obtains the required induction step. 
Proposition 5.4. Let $n \in \mathbb{N}$ and $H$ a strongly elliptic operator in non-divergence form (4) with complex coefficients $c_{i j}, c_{i}, c_{0} \in L_{\infty ; n}$.

Then $A^{\alpha} S_{t}^{\rho} L_{\hat{2}} \subset C^{\nu}(G) \cap L_{\infty}(G)$ for all $\alpha \in J_{n+1}(d), t>0, \rho \in \mathbb{R}$ and $v \in\langle 0,1\rangle$. Moreover, for all $v \in\langle 0,1\rangle$ and $M, \mu>0$ there exist $a, \omega>0$ such that

$$
\left\|A^{\alpha} S_{t}^{\rho} \varphi\right\|_{\infty} \leq a t^{-d / 4} t^{-|\alpha| / 2} e^{\omega\left(1+\rho^{2}\right) t}\|\varphi\|_{\hat{2}}
$$

and

$$
\left\|A^{\alpha} S_{t}^{\rho} \varphi\right\|_{C^{v}} \leq a t^{-d / 4} t^{-(|\alpha|+v) / 2} e^{\omega\left(1+\rho^{2}\right) t}\|\varphi\|_{\hat{2}}
$$

uniformly for all $H \in \mathscr{E}^{\text {nondiv }}(n, n, n, \mu, M), \varphi \in L_{\hat{2}}(G), t>0, \rho \in \mathbb{R}, \alpha \in J_{n+1}(d)$ and perturbation functions $\psi \in C_{c}^{\infty}(G)$ with $\left\|A^{\beta} \psi\right\|_{\infty} \leq 1$ for all $\beta \in J(d)$ with $1 \leq|\beta| \leq n+2$.

PROOF. It follows by interpolation from the $L_{\hat{2}}$-version of (7) and from (29) in [ElR6] that for all $p \in[2, \infty]$ there exist $a, \omega>0$ such that

$$
\left\|S_{t}^{\rho}\right\|_{\hat{2} \rightarrow \hat{p}} \leq a t^{-2^{-1} d\left(2^{-1}-p^{-1}\right)} e^{\omega\left(1+\rho^{2}\right) t}
$$

uniformly for all $\psi \in C_{c}^{\infty}(G)$ with $\left\|A_{i} \psi\right\|_{\infty} \leq 1$ for all $i \in\{1, \ldots, d\}$. Hence by Lemma 5.3 for each $p \in[2, \infty)$ there exist $a, \omega>0$ such that

$$
\left\|A^{\alpha} S_{t}^{\rho} \varphi\right\|_{\hat{p}: 1} \leq a t^{-2^{-1} d\left(2^{-1}-p^{-1}\right)} t^{-(|\alpha|+1) / 2} e^{\omega\left(1+\rho^{2}\right) t}\|\varphi\|_{\hat{2}}
$$

and

$$
\left\|A^{\alpha} S_{t}^{\rho} \varphi\right\|_{\hat{p}} \leq a t^{-2^{-1} d\left(2^{-1}-p^{-1}\right)} t^{-|\alpha| / 2} e^{\omega\left(1+\rho^{2}\right) t}\|\varphi\|_{\hat{2}}
$$

uniformly for all $\varphi \in L_{\hat{2}}, \alpha \in J_{n+1}(d), t>0, \rho \in \mathbb{R}$ and perturbation functions $\psi \in C_{c}^{\infty}(G)$ with $\left\|A^{\beta} \psi\right\|_{\infty} \leq 1$ for all $\beta \in J(d)$ with $1 \leq|\beta| \leq n+2$.

Next, if $p \in\langle d, \infty\rangle$ then there exists a $b>0$ such that

$$
\|\varphi\|_{\infty} \leq \varepsilon^{1-d / p}\|\varphi\|_{\hat{p} ; 1}+b \varepsilon^{-d / p}\|\varphi\|_{\hat{p}}
$$

uniformly for all $\varphi \in L_{\hat{p}: 1}$ and $\varepsilon \in\langle 0,1]$ by inequality [Rob, (IV.5.25)]. Setting $\varepsilon=t^{1 / 2}$ one obtains the bounds

$$
\left\|A^{\alpha} S_{t}^{\rho} \varphi\right\|_{\infty} \leq a t^{-d / 4} t^{-1} e^{\omega\left(1+\rho^{2}\right) t}\|\varphi\|_{\hat{2}}
$$

for all $\alpha \in J_{n+1}(d)$ and $t \leq 1$, and then, by the semigroup property, for all $t>0$ with increased values of $a$ and $\omega$.

Finally, the Hölder bounds follow from the Sobolev inequality

$$
\|\varphi\|_{C^{r}} \leq \varepsilon^{1-v-d / p}\|\varphi\|_{\hat{p} ; 1}+b_{p} \varepsilon^{-v-d / p}\|\varphi\|_{\hat{p}}
$$

for all $p>d(1-v)^{-1}$. The latter inequality follows as in [Rob, Section IV.5]. 
We can translate the bounds for non-divergence form operators into bounds for divergence form operators.

COROLlaRY 5.5. For all $n \in \mathbb{N}, v \in\langle 0,1\rangle$ and $M, \mu>0$ there exist a, $\omega>0$ such that

$$
\left\|A^{\alpha} S_{t}^{\rho} \varphi\right\|_{\infty} \leq a t^{-d / 4} t^{-|\alpha| / 2} e^{\omega\left(1+\rho^{2}\right) t}\|\varphi\|_{\hat{2}}
$$

and

$$
\left\|A^{\alpha} S_{t}^{\rho} \varphi\right\|_{C^{v}} \leq a t^{-d / 4} t^{-(|\alpha|+\nu) / 2} e^{\omega\left(1+\rho^{2}\right) t}\|\varphi\|_{\hat{2}}
$$

uniformly for all $H \in \mathscr{E}^{\text {div }}(n, n-1, n, n-1, \mu, M), \varphi \in L_{\hat{2}}(G), t>0, \rho \in \mathbb{R}$, $\alpha \in J_{n}(d)$ and perturbation functions $\psi \in C_{c}^{\infty}(G)$ with $\left\|A^{\beta} \psi\right\|_{\infty} \leq 1$ for all $\beta \in J(d)$ with $1 \leq|\beta| \leq n+1$.

This follows from Proposition 5.4 and (37).

The following is a non-divergence form version of Proposition 3.5. The proof is almost the same, with minor changes, which we leave to reader.

Proposition 5.6. Fix $N, N^{*} \in \mathbb{N}_{0}$ and $\nu, v^{*} \in\langle 0,1\rangle$. Next, let $N_{2} \in \mathbb{N}, N_{1}, N_{0} \in$ $\mathbb{N}_{0}, v_{2}, v_{1}, v_{0} \in[0,1\rangle, \mu>0$ and $N^{\prime} \in \mathbb{N}$. Suppose for all $M>0$ there exist $a>0$ and $\omega \geq 0$ such that $S_{t}^{\rho} C_{c}^{\infty}(G) \subset L_{2 ; N}, A^{\alpha} S_{t}^{\rho} C_{c}^{\infty}(G) \subset C^{\nu}(G) \cap L_{\infty}(G)$ for all $\alpha \in J_{N}(d)$, where $A$ is the $L_{2}$-derivative, $S_{t}^{* \rho} C_{c}^{\infty}(G) \subset L_{2: N^{*}}, A^{\beta} S_{t}^{* \rho} C_{c}^{\infty}(G) \subset C^{\nu^{*}}(G) \cap L_{\infty}(G)$ for all $\beta \in J_{N^{*}}(d)$,

$$
\begin{aligned}
\left\|A^{\alpha} S_{t}^{\rho} \varphi\right\|_{\infty} & \leq a t^{-d / 4} t^{-|\alpha| / 2} e^{\omega\left(1+\rho^{2}\right) t}\|\varphi\|_{\hat{2}}, \\
\left\|A^{\alpha} S_{t}^{\rho} \varphi\right\|_{C^{\nu}} & \leq a t^{-d / 4} t^{-(|\alpha|+\nu) / 2} e^{\omega\left(1+\rho^{2}\right) t}\|\varphi\|_{\hat{2}}, \\
\left\|A^{\beta} S_{t}^{* \rho} \varphi\right\|_{\infty} & \leq a t^{-d / 4} t^{-|\beta| / 2} e^{\omega\left(1+\rho^{2}\right) t}\|\varphi\|_{\hat{2}}, \\
\left\|A^{\beta} S_{t}^{* \rho} \varphi\right\|_{C^{*}} & \leq a t^{-d / 4} t^{-\left(|\beta|+\nu^{*}\right) / 2} e^{\omega\left(1+\rho^{2}\right) t}\|\varphi\|_{\hat{2}},
\end{aligned}
$$

uniformly for all $H \in \mathscr{E}^{\text {nondiv }}\left(N_{2}+\nu_{2}, N_{1}+v_{1}, N_{0}+v_{0}, \mu, M\right), \varphi \in C_{c}^{\infty}(G), \alpha \in$ $J_{N}\left(d^{\prime}\right), \beta \in J_{N^{*}}\left(d^{\prime}\right), \rho \in \mathbb{R}$ and $\psi \in C_{c}^{\infty}(G)$ with $\left\|A^{\gamma} \psi\right\|_{\infty} \leq 1$ for all $\gamma \in J(d)$ with $1 \leq|\gamma| \leq N^{\prime}$.

Then for all $\kappa>0$ and $M>0$ there exist $a, b>0$ and $\omega \geq 0$, such that for each $H \in \mathscr{E}^{\text {nondiv }}\left(N_{2}+v_{2}, N_{1}+v_{1}, N_{0}+v_{0}, \mu, M\right)$ the kernel $K$ of the semigroup $S$ generated by $H$ is $N$-times differentiable in the first variable, the derivatives are $N^{*}$ times differentiable with respect to the second variable, the derivatives are continuous,

$$
\left|\left(A^{\alpha} B^{\beta} K_{t}\right)(g ; h)\right| \leq a t^{-d / 2} t^{-(|\alpha|+|\beta|) / 2} e^{\omega t} e^{-b\left|g h^{-1}\right|^{2} t^{-1}}
$$

and

$$
\begin{aligned}
& \left|\left(A^{\alpha} B^{\beta} K_{t}\right)\left(k^{-1} g ; l^{-1} h\right)-\left(A^{\alpha} B^{\beta} K_{t}\right)(g ; h)\right| \\
& \quad \leq a t^{-d / 2} t^{-(|\alpha|+|\beta|) / 2} e^{\omega t}\left(\left(\frac{|k|}{t^{1 / 2}+\left|g h^{-1}\right|}\right)^{\nu}+\left(\frac{|l|}{t^{1 / 2}+\left|g h^{-1}\right|}\right)^{v^{*}}\right) e^{-b\left|g h^{-1}\right|^{2} t^{-1}}
\end{aligned}
$$


uniformly for all $\alpha \in J_{N}(d), \beta \in J_{N^{*}}(d), t>0, g, h \in G$ and $k, l \in G$ such that $|k|+|l| \leq \kappa t^{1 / 2}+2^{-1}\left|g h^{-1}\right|$.

THEOREM 5.7. Let $n \in \mathbb{N}$ and let $H$ be a strongly elliptic operator in nondivergence form (4) with complex coefficients $c_{i j}, c_{i}, c_{0} \in L_{\infty ; n}$. Then for all $\kappa>0$ and $v \in\langle 0,1\rangle$ there exist $a, b>0$ and $\omega \geq 0$, such that the kernel $K$ of the semigroup $S$ generated by $H$ is $(n+1)$-times differentiable in the first variable, the derivatives are $(n-1)$-times differentiable with respect to the second variable, the derivatives are continuous,

$$
\left|\left(A^{\alpha} B^{\beta} K_{t}\right)(g ; h)\right| \leq a t^{-d / 2} t^{-(|\alpha|+|\beta|) / 2} e^{\omega t} e^{-b\left|g h^{-1}\right|^{2} t^{-1}}
$$

and

$$
\begin{aligned}
& \left|\left(A^{\alpha} B^{\beta} K_{t}\right)\left(k^{-1} g ; l^{-1} h\right)-\left(A^{\alpha} B^{\beta} K_{t}\right)(g ; h)\right| \\
& \quad \leq a t^{-d / 2} t^{-(|\alpha|+|\beta|) / 2} e^{\omega t}\left(\frac{|k|+|l|}{t^{1 / 2}+\left|g h^{-1}\right|}\right)^{\nu} e^{-b\left|g h^{-1}\right|^{2} t^{-1}}
\end{aligned}
$$

uniformly for all $\alpha \in J_{n+1}(d), \beta \in J_{n-1}(d), t>0, g, h \in G$ and $k, l \in G$ such that $|k|+|l| \leq \kappa t^{1 / 2}+2^{-1}\left|g h^{-1}\right|$.

For all $\nu, \kappa, M$ and $\mu$ the constants $a, b$ and $\omega$ can be chosen uniformly for all $H \in \mathscr{E}^{\text {onondiv }}(n, n, n, \mu, M)$.

PROOF. This follows by a combination of the inclusion (38) with Propositions 3.4, 5.4, 5.6 and Corollary 5.5.

\section{Operators with $C^{n+v}$-coefficients, $n \in \mathbb{N}, 0<v<1$}

In this section we consider strongly elliptic operators in non-divergence form (4) with coefficients $c_{i j}, c_{i}, c_{0} \in C^{n+v}$ and again examine differentiability and smoothness of the corresponding kernels. The aim is to prove that the kernel is again almost $(n+2+v)$-times differentiable in the first variable. Precisely, for all $\varepsilon \in\langle 0, v\rangle$ we shall prove that the kernel is $(n+2)$-times differentiable and the $(n+2)$-nd derivative is Hölder continuous of order $\nu-\varepsilon$ in the first variable.

For all $k \in G$ introduce the operator $\delta_{k}=I-L(k)$ on the $L_{p}$-spaces with respect to the left-, or right-, Haar measure. Since $L(k) A_{i} L\left(k^{-1}\right)=d L\left(\operatorname{Ad}(k) a_{i}\right)$, it follows that $\left[\delta_{k}, A_{i}\right]=-\left[L(k), A_{i}\right]=d L\left((I-\operatorname{Ad}(k)) a_{i}\right) L(k)$. Moreover, $\|L(k) \varphi\|_{\hat{p}}=$ $\Delta(k)^{-1 / p}\|\varphi\|_{\hat{p}}$ and $k \mapsto \operatorname{Ad}(k)$ is a continuous representation of $G$ in the finitedimensional space $g$. From this it easily follows that for all $\alpha, \beta \in J(d), i \in$ $\{1, \ldots, d\}$ and $p \in[1, \infty]$ there exists an $a>0$ such that

$$
\left\|A^{\alpha}\left[\delta_{k}, A_{i}\right] A^{\beta} \varphi\right\|_{\hat{p}} \leq a|k|\|\varphi\|_{\hat{p}:|\alpha|+|\beta|+1}
$$


uniformly for all $\varphi \in L_{\hat{p} ;|\alpha|+|\beta|+1}$ and $k \in B(1)$. This is essential in the proof of the next lemma.

LEMMA 6.1. For all $n \in \mathbb{N}, v \in\langle 0,1\rangle, p \in\langle 1, \infty\rangle$ and $M, \mu>0$ there exist $a, \omega>0$ such that

$$
\left\|\delta_{k} A^{\alpha} S_{t}^{\rho}\right\|_{\hat{p} \rightarrow \hat{p}} \leq a|k|^{\nu} t^{-(|\alpha|+v) / 2} e^{\omega\left(1+\rho^{2}\right) t}
$$

uniformly for all $H \in \mathscr{E}^{\text {nondiv }}(n+\nu, n+\nu, n+\nu, \mu, M), \alpha \in J_{n+2}(d), k \in B(1)$, $t>0, \rho \in \mathbb{B}$ and perturbation functions $\psi \in C_{c}^{\infty}(G)$ with $\left\|A^{\beta} \psi\right\|_{\infty} \leq 1$ for all $\beta \in J(d)$ with $1 \leq|\beta| \leq n+2$.

ProOF. Let $H \in \mathscr{E}^{\text {nondiv }}(n+v, n+v, n+v, \mu, M)$. First by Lemma 5.3 and the $L_{\hat{p}}$-version of (6) one has

$$
\left\|\delta_{k} A^{\alpha} S_{t}^{\rho} \varphi\right\|_{\hat{p}} \leq\left(1+\Delta(k)^{-1 / p}\right)\left\|A^{\alpha} S_{t}^{\rho} \varphi\right\|_{\hat{p}} \leq c t^{-|\alpha| / 2} e^{\omega\left(1+\rho^{2}\right) t}\|\varphi\|_{\hat{p}}
$$

and

$$
\left\|\delta_{k} A^{\alpha} S_{t}^{\rho} \varphi\right\|_{\hat{p}} \leq d|k|\left\|A^{\alpha} S_{t}^{\rho} \varphi\right\|_{\hat{p} ; 1} \leq c|k| t^{-(|\alpha|+1) / 2} e^{\omega\left(1+\rho^{2}\right) t}\|\varphi\|_{\hat{p}}
$$

for all $\alpha \in J_{n+1}(d)$, for some $c>0$ which depends only on $n, M, \mu,\left\|A^{\gamma} \psi\right\|_{\infty}$ with $1 \leq|\gamma| \leq n+2$ and $p$. Hence, by interpolation,

$$
\left\|\delta_{k} A^{\alpha} S_{t}^{\rho} \varphi\right\|_{\hat{p}} \leq c|k|^{\nu} t^{-(|\alpha|+\nu) / 2} e^{\omega\left(1+\rho^{2}\right) t}\|\varphi\|_{\hat{p}}
$$

for all $\alpha \in J_{n+1}(d)$ and $k \in B(1)$.

Next we use the notation of Lemma 5.3, in particular the operators $H_{2}, H_{\rho}, V_{1}, V_{2}$ and $V_{3}$ for the proof of the bounds of the lemma if $|\alpha|=n+2$. Now, however, the operators $V_{1}, V_{2}$ and $V_{3}$ are non-divergence form operators with $C^{n+v}$-coefficients of order 0,1 and 1 , respectively.

Let $k \in B(1), l, m \in\{1, \ldots, d\}, \alpha \in J(d),|\alpha|=n, t>0, \rho \in \mathbb{R}$ and $\varphi \in L_{\hat{p} ; n+2}$. We estimate $\left\|\delta_{k} A_{l} A_{m} A^{\alpha} S_{t}^{\rho} \varphi\right\|_{\hat{p}}$. By Corollary 5.2 there exist $a, \lambda_{0}>0$, depending only on $M, \mu$ and $p$, such that $\|\eta\|_{\hat{p}: 2} \leq a\left\|\left(\lambda_{0} I+H_{2}\right) \eta\right\|_{\hat{p}}$ uniformly for all $\eta \in L_{\hat{p} ; 2}$. Therefore,

$$
\begin{aligned}
\left\|\delta_{k} A_{l} A_{m} A^{\alpha} S_{t}^{\rho} \varphi\right\|_{\hat{p}} \leq & \left\|A_{l} A_{m} \delta_{k} A^{\alpha} S_{t}^{\rho} \varphi\right\|_{\hat{p}}+\left\|\left[\delta_{k}, A_{l} A_{m}\right] A^{\alpha} S_{t}^{\rho} \varphi\right\|_{\hat{p}} \\
\leq & a\left\|\left(\lambda_{0} I+H_{2}\right) \delta_{k} A^{\alpha} S_{t}^{\rho} \varphi\right\|_{\hat{p}}+\left\|\left[\delta_{k}, A_{l} A_{m}\right] A^{\alpha} S_{t}^{\rho} \varphi\right\|_{\hat{p}} \\
\leq & a \lambda_{0}\left\|\delta_{k} A^{\alpha} S_{t}^{\rho} \varphi\right\|_{\hat{p}}+a\left\|H_{2} \delta_{k} A^{\alpha} S_{t}^{\rho} \varphi\right\|_{\hat{p}} \\
& \quad+\left\|A_{l}\left[\delta_{k}, A_{m}\right] A^{\alpha} S_{t}^{\rho} \varphi\right\|_{\hat{p}}+\left\|\left[\delta_{k}, A_{l}\right] A_{m} A^{\alpha} S_{t}^{\rho} \varphi\right\|_{\hat{p}} .
\end{aligned}
$$


Next,

$$
\begin{aligned}
& H_{2} \delta_{k} A^{\alpha} S_{t}^{\rho} \varphi \\
& =\delta_{k} H_{2} A^{\alpha} S_{t}^{\rho} \varphi+\left[H_{2}, \delta_{k}\right] A^{\alpha} S_{t}^{\rho} \varphi \\
& =\delta_{k} A^{\alpha} H_{2} S_{t}^{\rho} \varphi+\delta_{k}\left[H_{2}, A^{\alpha}\right] S_{t}^{\rho} \varphi+\left[H_{2}, \delta_{k}\right] A^{\alpha} S_{t}^{\rho} \varphi \\
& =\delta_{k} A^{\alpha} H_{\rho} S_{t}^{\rho} \varphi+\rho^{2} \delta_{k} A^{\alpha} V_{1} S_{t}^{\rho} \varphi+\rho \delta_{k} A^{\alpha} V_{2} S_{t}^{\rho} \varphi+\delta_{k} A^{\alpha} V_{3} S_{t}^{\rho} \varphi+\delta_{k}\left[H_{2}, A^{\alpha}\right] S_{t}^{\rho} \varphi \\
& \quad-\sum_{i, j=1}^{d}\left[c_{i j}, \delta_{k}\right] A_{i} A_{j} A^{\alpha} S_{t}^{\rho} \varphi+\sum_{i, j=1}^{d} c_{i j}\left[\delta_{k}, A_{i}\right] A_{j} A^{\alpha} S_{t}^{\rho} \varphi \\
& \quad+\sum_{i, j=1}^{d} c_{i j} A_{i}\left[\delta_{k}, A_{j}\right] A^{\alpha} S_{t}^{\rho} \varphi .
\end{aligned}
$$

So

$$
\begin{aligned}
\| \delta_{k} A_{l} A_{m} A^{\alpha} & S_{t}^{\rho} \varphi \|_{\hat{p}} \\
\leq a & \lambda_{0}\left\|\delta_{k} A^{\alpha} S_{t}^{\rho} \varphi\right\|_{\hat{p}}+\left\|A_{l}\left[\delta_{k}, A_{m}\right] A^{\alpha} S_{t}^{\rho} \varphi\right\|_{\hat{p}}+\left\|\left[\delta_{k}, A_{l}\right] A_{m} A^{\alpha} S_{t}^{\rho} \varphi\right\|_{\hat{p}} \\
& +a\left\|\delta_{k} A^{\alpha} H_{\rho} S_{t}^{\rho} \varphi\right\|_{\hat{p}}+a \rho^{2}\left\|\delta_{k} A^{\alpha} V_{1} S_{t}^{\rho} \varphi\right\|_{\hat{p}}+a|\rho|\left\|\delta_{k} A^{\alpha} V_{2} S_{t}^{\rho} \varphi\right\|_{\hat{p}} \\
& +a\left\|\delta_{k} A^{\alpha} V_{3} S_{t}^{\rho} \varphi\right\|_{\hat{p}}+a\left\|\delta_{k}\left[H_{2}, A^{\alpha}\right] S_{t}^{\rho} \varphi\right\|_{\hat{p}} \\
& +a \sum_{i, j=1}^{d}\left\|\left[c_{i j}, \delta_{k}\right] A_{i} A_{j} A^{\alpha} S_{t}^{\rho} \varphi\right\|_{\hat{p}}+a \sum_{i, j=1}^{d}\left\|c_{i j}\left[\delta_{k}, A_{i}\right] A_{j} A^{\alpha} S_{t}^{\rho} \varphi\right\|_{\hat{p}} \\
& +a \sum_{i, j=1}^{d}\left\|c_{i j} A_{i}\left[\delta_{k}, A_{j}\right] A^{\alpha} S_{t}^{\rho} \varphi\right\|_{\hat{p}},
\end{aligned}
$$

a sum of eleven terms. We give the details of the estimates of four terms, the other are similar, or follow easily from Lemma 5.3 and (42).

The fourth term can be estimated as follows. By (41) and (43) it follows that

$$
\left\|\delta_{k} A^{\alpha} H_{\rho} S_{t}^{\rho} \varphi\right\|_{\hat{p}} \leq\left\|\delta_{k} A^{\alpha} S_{t / 2}^{\rho}\right\|_{\hat{p} \rightarrow \hat{p}}\left\|H_{\rho} S_{t / 2}^{\rho} \varphi\right\|_{\hat{p}} \leq c|k|^{\nu} t^{-(n+2+v) / 2} e^{\omega\left(1+\rho^{2}\right) t}\|\varphi\|_{\hat{p}}
$$

for a suitable $c>0$.

The fifth term, $\rho^{2}\left\|\delta_{k} A^{\alpha} V_{1} S_{t}^{\rho} \varphi\right\|_{\hat{p}}$, can be estimated by a finite sum of terms of the form $\rho^{2}\left\|\delta_{k} A^{\alpha} \tau S_{t}^{\rho} \varphi\right\|_{\hat{p}}$, with $\tau \in C^{n+\nu}$. Then Leibniz' rule together with (5) gives

$$
\begin{aligned}
\rho^{2}\left\|\delta_{k} A^{\alpha} \tau S_{t}^{\rho} \varphi\right\|_{\hat{p}} & \leq \sum_{|\beta|+|\gamma|=n} \sum_{j=0}^{1} \rho^{2}\left\|\delta_{k}^{j} A^{\beta} \tau\right\|_{\infty}\left\|\delta_{k}^{1-j} A^{\gamma} S_{t}^{\rho} \varphi\right\|_{\hat{p}} \\
& \leq \sum_{|\beta|+|\gamma|=n} \sum_{j=0}^{1} \rho^{2} a|k|^{j \nu}\left(|k| t^{-1 / 2}\right)^{v(1-j)} t^{-|\gamma| / 2} e^{\omega\left(1+\rho^{2}\right) t}\|\varphi\|_{\hat{p}} \\
& \leq a^{\prime} \rho^{2}|k|^{\nu} t^{-(n+\nu) / 2} e^{\omega\left(1+\rho^{2}\right) t}\|\varphi\|_{\hat{p}} \leq a^{\prime \prime}|k|^{\nu} t^{-(n+2+\nu) / 2} e^{\omega^{\prime}\left(1+\rho^{2}\right) t}\|\varphi\|_{\hat{p}},
\end{aligned}
$$


where we have used (43).

The sixth term can be estimated by a sum of terms of the form $|\rho|\left\|\delta_{k} A^{\alpha} \tau A_{i} S_{t}^{\rho} \varphi\right\|_{\hat{p}}$, with $\tau \in C^{n+\nu}$. By Leibniz' rule one deduces as for the fifth term that

$$
|\rho|\left\|\delta_{k} A^{\alpha} \tau A_{i} S_{t}^{\rho} \varphi\right\|_{\hat{p}} \leq a|k|^{\nu} t^{-(n+2+v) / 2} e^{\omega\left(1+\rho^{2}\right) t}\|\varphi\|_{\hat{p}} .
$$

All the other terms can be estimated with a better $t$ singularity. For example, in the second term one has

$$
\begin{aligned}
\left\|A_{l}\left[\delta_{k}, A_{m}\right] A^{\alpha} S_{t}^{\rho} \varphi\right\|_{\hat{p}} & \leq a|k|\left\|S_{t}^{\rho} \varphi\right\|_{\hat{p}: n+2} \\
& \leq a^{\prime}|k| t^{-(n+2) / 2} e^{\omega\left(1+\rho^{2}\right) t}\|\varphi\|_{\hat{p}} \leq a^{\prime \prime}|k|^{\nu} t^{-(n+2+\nu) / 2} e^{\omega^{\prime}\left(1+\rho^{2}\right) t}\|\varphi\|_{\hat{p}},
\end{aligned}
$$

where we have used (42), Lemma 5.3 and $|k| \leq|k|^{\nu}$ for $k \in B(1)$. We leave the other parts to the reader.

PROPOSITION 6.2. Let $n \in \mathbb{N}, v \in\langle 0,1\rangle$ and let $H$ be a strongly elliptic operator in non-divergence form (4) with complex coefficients $c_{i j}, c_{i}, c_{0} \in C^{n+\nu}$. Let $\gamma \in\langle 0, v\rangle$. Then $A^{\alpha} S_{t}^{\rho} L_{\hat{2}} \subset C^{\gamma}(G) \cap L_{\infty}(G)$ for all $\alpha \in J_{n+2}(d), t>0, \rho \in \mathbb{R}$.

Moreover, for all $M, \mu>0$ there exist a, $\omega>0$ such that

$$
\left\|A^{\alpha} S_{t}^{\rho} \varphi\right\|_{\infty} \leq a t^{-d / 4} t^{-|\alpha| / 2} e^{\omega\left(1+\rho^{2}\right) t}\|\varphi\|_{\hat{2}}
$$

and

$$
\left\|A^{\alpha} S_{t}^{\rho} \varphi\right\|_{C^{\gamma}} \leq a t^{-d / 4} t^{-(|\alpha|+\gamma) / 2} e^{\omega\left(1+\rho^{2}\right) t}\|\varphi\|_{\hat{2}}
$$

uniformly for all $H \in \mathscr{E}^{\text {nondiv }}(n+\nu, n+\nu, n+\nu, \mu, M), \varphi \in L_{\hat{2}}(G), t>0, \rho \in \mathbb{R}$, $\alpha \in J_{n+2}(d)$ and perturbation functions $\psi \in C_{c}^{\infty}(G)$ with $\left\|A^{\beta} \psi\right\|_{\infty} \leq 1$ for all $\beta \in J(d)$ with $1 \leq|\beta| \leq n+2$.

PROOF. Arguing as in the proof of Proposition 5.4 it follows that for all $\tau \in\langle 0,1\rangle$ and $M, \mu>0$ there exist $a, \omega>0$ such that

$$
\|\| \delta_{k} A^{\alpha} S_{i}^{\rho} \varphi\left\|_{C^{r}} \leq a|k|^{\nu} t^{-d / 4} t^{-(|\alpha|+\nu+r) / 2} e^{\omega\left(1+\rho^{2}\right) t}\right\| \varphi \|_{\hat{2}}
$$

uniformly for all $H \in \mathscr{E}^{\text {nondiv }}(n+v, n+v, n+v, \mu, M), \varphi \in L_{\hat{2}}(G), k \in B(1), t>0$, $\rho \in \mathbb{R}, \alpha \in J_{n+1}(d)$ and perturbation functions $\psi \in C_{c}^{\infty}(G)$ with $\left\|A^{\beta} \psi\right\|_{\infty} \leq 1$ for all $\beta \in J(d)$ with $1 \leq|\beta| \leq n+2$. So

$$
\begin{aligned}
\left\|(I-L(g))(I-L(k)) A^{\alpha} S_{t}^{\rho} \varphi\right\|_{\infty} & \leq a|g|^{\tau}|k|^{v} t^{-d / 4} t^{-(|\alpha|+v+\tau) / 2} e^{\omega\left(1+\rho^{2}\right) r}\|\varphi\|_{\hat{2}} \\
& \leq a s^{v+\tau} t^{-d / 4} t^{-(|\alpha|+v+\tau) / 2} e^{\omega\left(1+\rho^{2}\right) t}\|\varphi\|_{\hat{2}}
\end{aligned}
$$


uniformly for all $s \in\langle 0,1]$ and $g, k \in B(s)$. As a consequence, using the uniform Lipschitz spaces in [ElR1, p. 185] and [ElR1, Theorem 3.2], one then deduces that $A^{\alpha} S_{t}^{\rho} \varphi \in\left(L_{\infty}, L_{\infty ; 2}\right)_{(v+\tau) / 2, \infty ; K}$ and

$$
\left\|A^{\alpha} S_{t}^{\rho} \varphi\right\|_{\left(L_{x}, L_{x: 2}\right)_{(v+r) / 2 . x: K}} \leq a t^{-d / 4} t^{-(|\alpha|+\nu+\tau) / 2} e^{\omega\left(1+\rho^{2}\right) t}\|\varphi\|_{\hat{2}} .
$$

Choosing $\tau=1+\gamma-v$ and using the identity $\left(L_{\infty}, L_{\infty ; 2}\right)_{(1+\gamma) / 2, \infty ; K}=\left(L_{\infty}, L_{\infty ; 1}\right)_{\gamma, \infty ; K ; 1}$ $=C^{1+\gamma}$, with equivalent norms (see [EIR1, Theorem 2.1.III]), it follows that $A^{\alpha} S_{t}^{\rho} \varphi \in$ $L_{\infty ; 1}$ and

$$
\left\|A_{i} A^{\alpha} S_{t}^{\rho} \varphi\right\|_{C^{\gamma}} \leq a t^{-d / 4} t^{-(|\alpha|+1+\gamma) / 2} e^{\omega\left(1+\rho^{2}\right) t}\|\varphi\|_{\hat{2}}
$$

uniformly for all $\alpha \in J_{n+1}(d)$ and $i \in\{1, \ldots, d\}$.

Finally, interpolation (see [EIR1, Proposition 4.3.I]) between the bounds (45) and the first bounds of Proposition 5.4 then gives the bounds (44).

This proposition immediately leads to the next theorem.

THEOREM 6.3. Let $n \in \mathbb{N}, v \in\langle 0,1\rangle$ and let $H$ be a strongly elliptic operator in non-divergence form (4) with complex coefficients $c_{i j}, c_{i}, c_{0} \in C^{n+v}$. Then for all $\kappa>0$ and $\gamma \in\langle 0, v\rangle$ there exist $a, b>0$ and $\omega \geq 0$, such that the kernel $K$ of the semigroup $S$ generated by $H$ is $(n+2)$-times differentiable in the first variable, the derivatives are n-times differentiable with respect to the second variable, the derivatives are continuous,

$$
\left|\left(A^{\alpha} B^{\beta} K_{t}\right)(g ; h)\right| \leq a t^{-d / 2} t^{-(|\alpha|+|\beta|) / 2} e^{\omega t} e^{-b\left|g h^{-1}\right|^{2} t^{-1}}
$$

and

$$
\begin{aligned}
& \left|\left(A^{\alpha} B^{\beta} K_{t}\right)\left(k^{-1} g ; l^{-1} h\right)-\left(A^{\alpha} B^{\beta} K_{t}\right)(g ; h)\right| \\
& \quad \leq a t^{-d / 2} t^{-(|\alpha|+|\beta|) / 2} e^{\omega t}\left(\frac{|k|+|l|}{t^{1 / 2}+\left|g h^{-1}\right|}\right)^{\gamma} e^{-b\left|g h^{-1}\right|^{2} t^{-1}}
\end{aligned}
$$

uniformly for all $\alpha \in J_{n+2}(d), \beta \in J_{n}(d), t>0, g, h \in G$ and $k, l \in G$ such that $|k|+|l| \leq \kappa t^{1 / 2}+2^{-1}\left|g h^{-1}\right|$. Moreover, for all $\gamma, \kappa, M$ and $\mu$ the constants $a, b$ and $\omega$ can be chosen uniformly for all $H \in \mathscr{E}^{\text {nondiv }}(n+v, n+v, n+v, \mu, M)$.

Finally we discuss the regularity of the operators $(\lambda I+H)^{\delta}$ on $L_{p}$ if all the coefficients are in $C^{n+v}$.

THEOREM 6.4. Let $n \in \mathbb{N}, v \in\langle 0,1\rangle$ and let $H$ be a strongly elliptic operator in non-divergence form (4) with complex coefficients $c_{i j}, c_{i}, c_{0} \in C^{n+\nu}$. Then

$$
D\left((\lambda I+H)^{(n+2+\gamma) / 2}\right)=L_{p: n+2+\gamma}
$$


for all large $\lambda, \gamma \in\langle 0, v\rangle$ and $p \in\langle 1, \infty\rangle$, with equivalent norms.

Moreover, for all $M, \mu>0$ there exists a $\lambda_{0}>0$ such that for all $\gamma \in\langle 0, \nu\rangle$ and $p \in\langle 1, \infty\rangle$ there exists an $a>0$ and for all $\lambda \geq \lambda_{0}$ and $a_{\lambda}>0$ such that

$$
a_{\lambda}\left\|(\lambda I+H)^{(n+2+\gamma) / 2} \varphi\right\|_{p} \leq\|\varphi\|_{p ; n+2+\gamma} \leq a\left\|(\lambda I+H)^{(n+2+\gamma) / 2} \varphi\right\|_{p}
$$

uniformly for all $H \in \mathscr{E}^{\text {nondiv }}(n+\nu, n+\nu, n+\nu, \mu, M)$ and $\varphi \in L_{p ; n+2+\gamma}$.

Similar conclusions are valid on the $L_{\hat{p}}$-spaces.

PROOF. For the upper bounds it suffices to prove the bounds for one $\lambda$, dependent on $p$. It follows from Corollary 5.2 that $D\left((\lambda I+H)^{(n+2) / 2}\right)=L_{p ; n+2}$, with equivalent norms. So by interpolation the operators $\left(I+H_{L}\right)^{\delta}(\lambda I+H)^{-\delta}$ are bounded on $L_{p}$ for all $\delta \in[0,(n+2) / 2]$. Moreover, the operator $(\lambda I+H)^{-(n+2) / 2}$ is bounded from $L_{p}$ into $L_{p ; n+2}$, if $\lambda$ is large enough. We have to distinguish between even and odd $n$ in the proof. We only prove the case for odd $n$, the other case is slightly easier and is left to the reader.

Let $m \in \mathbb{N}$ be such that $n+1=2 m$. Write $(\lambda I+H)^{m}=\sum_{\alpha \in J_{2 m}(d)} f_{\alpha} A^{\alpha}$, with $f_{\alpha} \in C^{1+v}$. For all $\alpha \in J_{2 m}(d)$ there exists by Proposition 2.3.IV a continuous operator $F_{\alpha}: L_{p ; 1} \rightarrow L_{p}$ such that

$$
F_{\alpha} \varphi=\left[f_{\alpha},\left(I+H_{L}\right)^{(1+\gamma) / 2}\right] \varphi
$$

for all $\varphi \in L_{p, 1+\gamma}$. Moreover, by [EIR2, Theorem 3.13], there exists a continuous operator $D_{\alpha}: L_{p ; 2 m+\gamma} \rightarrow L_{p}$ such that

$$
D_{\alpha} \varphi=\left[A^{\alpha},\left(I+H_{L}\right)^{(1+\gamma) / 2}\right] \varphi
$$

for all $\varphi \in L_{p: 2 m+1+\gamma}$. Then

$$
\begin{gathered}
\left(\psi,(\lambda I+H)^{m}\left(I+H_{L}\right)^{(1+\gamma) / 2} \varphi\right)-\left(\psi,\left(I+H_{L}\right)^{(1+\gamma) / 2}(\lambda I+H)^{m} \varphi\right) \\
=\sum_{\alpha \in J_{2 m}(d)}\left(\psi, F_{\alpha} A^{\alpha} \varphi\right)+\left(\psi, f_{\alpha} D_{\alpha} \varphi\right)
\end{gathered}
$$

for all $\varphi \in L_{p ; 2 m+1+\gamma}$ and $\psi \in L_{q}$. Now $H^{*} \in \mathscr{E}^{\mathrm{div}}(n+\nu, P, n-1+\nu, n+\nu, \mu$, $(d+1) M)$ for all $P \in[0, \infty\rangle$, by $(38)$. So $D\left(\left(\lambda I+H^{*}\right)^{m}\right) \subset D\left(\left(\lambda I+H^{*}\right)^{1 / 2}\right)=L_{q ; 1}$ by Corollary 4.8 . Therefore

$$
\left(\left(\lambda I+H^{*}\right)^{m} \psi,\left(I+H_{L}\right)^{(1+\gamma) / 2} \varphi\right)-\left(\left(I+H_{L}\right)^{1 / 2} \psi,\left(I+H_{L}\right)^{\gamma / 2}(\lambda I+H)^{m} \varphi\right)
$$

$$
=\sum_{\alpha \in J_{2 m}(d)}\left(\psi, F_{\alpha} A^{\alpha} \varphi\right)+\left(\psi, f_{\alpha} D_{\alpha} \varphi\right)
$$


for all $\varphi \in L_{2 m+1+\gamma}$ and $\psi \in D\left(\left(\lambda I+H^{*}\right)^{m}\right)$. But $\varphi \mapsto\left(I+H_{L}\right)^{\gamma / 2}(\lambda I+H)^{m} \varphi$ is continuous from $L_{p ; 2 m+1}$ into $L_{p}$ (Corollary 5.2) and $L_{p ; 2 m+1+\gamma}$ is dense in $L_{p ; 2 m+1}$. Hence (46) is valid for all $\varphi \in L_{p: 2 m+1}$ and $\psi \in D\left(\left(\lambda I+H^{*}\right)^{m}\right)$.

Now let $\varphi \in D\left((\lambda I+H)^{(n+2+\gamma) / 2}\right)$ and $\psi \in L_{q ; n+2+\gamma}$. Then $\left(I+H_{L}\right)^{m} \psi \in L_{q}$ and $\left(\lambda I+H^{*}\right)^{-m}\left(I+H_{L}\right)^{m} \psi \in D\left(\left(\lambda I+H^{*}\right)^{m}\right)$. Moreover, $\varphi \in D\left((\lambda I+H)^{(2 m+1) / 2}\right)=$ $L_{p: 2 m+1}$. Therefore

$$
\begin{aligned}
\mid((\lambda I+ & \left.\left.H_{L}\right)^{(n+2+\gamma) / 2} \psi, \varphi\right) \mid \\
= & \left|\left(\left(\lambda I+H^{*}\right)^{m}\left(\lambda I+H^{*}\right)^{-m}\left(I+H_{L}\right)^{m} \psi,\left(I+H_{L}\right)^{(1+\gamma) / 2} \varphi\right)\right| \\
\leq & \left|\left(\left(I+H_{L}\right)^{1 / 2}\left(\lambda I+H^{*}\right)^{-m}\left(I+H_{L}\right)^{m} \psi,\left(I+H_{L}\right)^{\gamma / 2}(\lambda I+H)^{m} \varphi\right)\right| \\
& \quad+\sum_{\alpha \in J_{2 m}(d)}\left|\left(\left(\lambda I+H^{*}\right)^{-m}\left(I+H_{L}\right)^{m} \psi, F_{\alpha} A^{\alpha} \varphi\right)\right| \\
& +\left|\left(\left(\lambda I+H^{*}\right)^{-m}\left(I+H_{L}\right)^{m} \psi, f_{\alpha} D_{\alpha} \varphi\right)\right| .
\end{aligned}
$$

We estimate the contributions of the three types of terms. The first can be bounded by

$$
\begin{aligned}
\left|\left(\left(I+H_{L}\right)^{1 / 2}\left(\lambda I+H^{*}\right)^{-m}\left(I+H_{L}\right)^{m} \psi,\left(I+H_{L}\right)^{\gamma / 2}(\lambda I+H)^{m} \varphi\right)\right| \\
=\mid\left(\psi,\left(\left(I+H_{L}\right)^{m}(\lambda I+H)^{-m}\right)\left(\left(I+H_{L}\right)^{(1+\gamma) / 2}(\lambda I+H)^{-(1+\gamma) / 2}\right)\right. \\
\left.\quad \cdot(\lambda I+H)^{(n+2+\gamma) / 2} \varphi\right) \mid \\
\leq\|\psi\|_{q}\left\|\left(I+H_{L}\right)^{m}(\lambda I+H)^{-m}\right\|_{p \rightarrow p} \\
\cdot\left\|\left(I+H_{L}\right)^{(1+\gamma) / 2}(\lambda I+H)^{-(1+\gamma) / 2}\right\|_{p \rightarrow p}\left\|(\lambda I+H)^{(n+2+\gamma) / 2} \varphi\right\|_{p} .
\end{aligned}
$$

For all $\alpha \in J_{2 m}(d)$ one has for the second term

$$
\begin{aligned}
\left|\left(\left(\lambda I+H^{*}\right)^{-m}\left(I+H_{L}\right)^{m} \psi, F_{\alpha} A^{\alpha} \varphi\right)\right| \\
=\mid\left(\psi,\left(\left(I+H_{L}\right)^{m}(\lambda I+H)^{-m}\right)\left(F_{\alpha} A^{\alpha}(\lambda I+H)^{-(2 m+1) / 2}\right)\right. \\
\left.\quad \cdot(\lambda I+H)^{-\gamma / 2}(\lambda I+H)^{(n+2+\gamma) / 2} \varphi\right) \mid \\
\leq\|\psi\|_{q}\left\|\left(I+H_{L}\right)^{m}(\lambda I+H)^{-m}\right\|_{p \rightarrow p}\left\|F_{\alpha}\right\|_{L_{p: I} \rightarrow L_{p}}\left\|(\lambda I+H)^{-(2 m+1) / 2}\right\|_{L_{p} \rightarrow L_{p: 2 m+1}} \\
\cdot\left\|(\lambda I+H)^{-\gamma / 2}\right\|_{p \rightarrow p}\left\|(\lambda I+H)^{(n+2+\gamma) / 2} \varphi\right\|_{p} .
\end{aligned}
$$

Finally,

$$
\begin{aligned}
\mid((\lambda I+ & \left.\left.H^{*}\right)^{-m}\left(I+H_{L}\right)^{m} \psi, f_{\alpha} D_{\alpha} \varphi\right) \mid \\
= & \mid\left(\psi,\left(\left(I+H_{L}\right)^{m}(\lambda I+H)^{-m}\right) f_{\alpha}\left(D_{\alpha}(\lambda I+H)^{-(2 m+1) / 2}\right)\right. \\
& \left.\cdot(\lambda I+H)^{-\gamma / 2}(\lambda I+H)^{(n+2+\gamma) / 2} \varphi\right) \mid \\
\leq & \|\psi\|_{q}\left\|\left(I+H_{L}\right)^{m}(\lambda I+H)^{-m}\right\|_{p \rightarrow p}\left\|f_{\alpha}\right\|_{\infty}\left\|D_{\alpha}\right\|_{L_{p: 2 m+1} \rightarrow L_{p}} \\
& \cdot\left\|(\lambda I+H)^{-(2 m+1) / 2}\right\|_{L_{p} \rightarrow L_{p: 3 m+1}}\left\|(\lambda I+H)^{-\gamma / 2}\right\|_{p \rightarrow p}\left\|(\lambda I+H)^{(n+2+\gamma) / 2} \varphi\right\|_{p}
\end{aligned}
$$


Hence

$$
\left|\left(\left(\lambda I+H_{L}\right)^{(n+2+\gamma) / 2} \psi, \varphi\right)\right| \leq c\|\psi\|_{q}\left\|(\lambda I+H)^{(n+2+\gamma) / 2} \varphi\right\|_{p}
$$

for a suitably chosen $c>0$ and $\varphi \in D\left(\left(\left(\lambda I+H_{L}\right)^{(n+2+\gamma) / 2}\right)^{*}\right)=L_{p ; n+2+\gamma}$. Thus $D\left((\lambda I+H)^{(n+2+\gamma) / 2}\right) \subseteq L_{p ; n+2+\gamma}$.

Conversely, by Corollary 5.2 and interpolation, again, there exists an $a_{\lambda}>0$ such that $\left\|(\lambda I+H)^{(n+\gamma) / 2}\left(I+H_{L}\right)^{-(n+\gamma) / 2} \varphi\right\|_{p} \leq a_{\lambda}\|\varphi\|_{p}$ uniformly for all $\varphi \in L_{p}$. Then by duality $\left\|\left(I+H_{L}\right)^{-(n+\gamma) / 2}\left(\lambda I+H^{*}\right)^{(n+\gamma) / 2} \psi\right\|_{q} \leq a_{\lambda}\|\psi\|_{q}$ uniformly for all $\psi \in L_{q}$. Next let $\psi \in D\left(\left(\lambda I+H^{*}\right)^{(n+2+\gamma) / 2}\right)$ and $\varphi \in L_{p ; n+2+\gamma}$. Then

$$
\begin{aligned}
\mid((\lambda I & \left.\left.+H^{*}\right)^{(n+2+\gamma) / 2} \psi, \varphi\right) \mid \\
& =\left|\left(\left(I+H_{L}\right)^{-(n+\gamma) / 2}\left(\lambda I+H^{*}\right)^{(n+\gamma) / 2} \psi,\left(I+H_{L}\right)^{(n+\gamma) / 2}(\lambda I+H) \varphi\right)\right| \\
& \leq a_{\lambda}\|\psi\|_{q}\|(\lambda I+H) \varphi\|_{p: n+\gamma}
\end{aligned}
$$

by the Cauchy-Schwarz inequality. Since all the coefficients of $H$ belong to $C^{n+\gamma}$ it follows from Proposition 2.3.II that there exist $a, a^{\prime}>0$, depending only on $M$, such that $\|H \varphi\|_{p ; n+\gamma} \leq a \sup _{\alpha \in J_{2}(d)}\left\|A^{\alpha} \varphi\right\|_{p ; n+\gamma} \leq a^{\prime}\|\varphi\|_{p ; n+2+\gamma}$. Combining these estimates then gives

$$
\left|\left(\left(\lambda I+H^{*}\right)^{(n+2+\gamma) / 2} \psi, \varphi\right)\right| \leq a_{\lambda}\left(\lambda+a^{\prime}\right)\|\varphi\|_{p ; n+2+\gamma}\|\psi\|_{q} .
$$

So $\varphi \in D\left(\left(\left(\lambda I+H^{*}\right)^{(n+2+\gamma) / 2}\right)^{*}\right)=D\left((\lambda I+H)^{(n+2+\gamma) / 2}\right)$ and $\left\|(\lambda I+H)^{(n+2+\gamma) / 2} \varphi\right\|_{p} \leq$ $a_{\lambda}\left(\lambda+a^{\prime}\right)\|\varphi\|_{p ; n+2+\gamma}$. This completes the proof of the theorem.

\section{Appendix 1: Singular integration}

The standard theory of singular integration, [CoW, Sections III.1 and III.2], is developed for homogeneous spaces with the doubling property. In particular it does not apply directly to spaces which only have a local doubling property such as nonunimodular Lie groups with Haar measure and the metric associated with an algebraic basis. Nevertheless the arguments underlying the theory can be adapted to the general situation.

THEOREM A.1. Let $X$ be a space with metric $\rho$ and Borel measure $\mu$. Assume that for each bounded set $X_{0} \subset X$ and $M>0$ there exists a $c_{d}>0$ such that

$$
0<\mu(B(x ; 2 r)) \leq c_{d} \mu(B(x ; r))<\infty
$$

uniformly for all $x \in X_{0}$ and $r \in\langle 0, M]$. Let $\kappa \in L_{2}(X \times X, d \mu \otimes d \mu)$ and suppose that supp $\kappa$ is bounded in $X \times X$. Define the operator $T: L_{2}(X) \rightarrow L_{2}(X)$ by

$$
(T \varphi)(x)=\int_{X} d \mu(x) \kappa(x ; y) \varphi(y) .
$$


Suppose there exists a $c_{1}>0$ such that $\|T \varphi\|_{2} \leq c_{1}\|\varphi\|_{2}$ for all $\varphi \in L_{2}(X)$. Moreover, suppose there exist $c_{2}, c_{3}>1$ such that

$$
\sup _{y, y_{0} \in X} \int_{\Omega\left(y, y_{0}\right)} d \mu(x)\left|\kappa(x ; y)-\kappa\left(x ; y_{0}\right)\right| \leq c_{3}
$$

where $\Omega\left(y, y_{0}\right)=\left\{x \in X: \rho(x ; y)>c_{2} \rho\left(y ; y_{0}\right)\right\}$. Then there exists a constant $A>0$ such that

$$
\mu(\{x \in X:|(T \varphi)(x)|>\gamma\}) \leq A \gamma^{-1}\|\varphi\|_{1}
$$

uniformly for all $\varphi \in L_{1}(X) \cap L_{2}(X)$ and $\gamma>0$. The constant $A$ depends on the constants $c_{1}, c_{2}$ and $c_{3}$, a suitable doubling constant $c_{d}$ and the set $Y$, but is independent of $\kappa$.

PROOF. The proof is very similar to the proof in [CoW, Sections III.1 and III.2] for homogeneous spaces which have the doubling property. If there were a measurable set $Y \subset X$ such that $\operatorname{supp} \kappa \subseteq Y \times Y$ and the set $Y$, with the relative metric and measure, had the doubling property then one could apply [CoW, Theorem III.2.4] directly. The problem, however, is that subsets often fail to have the doubling property, even if $X$ itself has the doubling property. This depends crucially on the geometric details of the subsets.

In the proof of this theorem one has to make the functions local, and exploit the localized doubling property, to obtain a Calderón-Zygmund decomposition, as we show below with some care.

There exist $x_{0} \in X$ and $R_{0}>0$ such that $\operatorname{supp} \kappa \subset B\left(x_{0} ; R_{0}\right) \times B\left(x_{0} ; R_{0}\right)$. Let $c_{d}>0$ be such that

$$
0<\mu(B(x ; 2 r)) \leq c_{d} \mu(B(x ; r))<\infty
$$

uniformly for all $x \in B\left(x_{0} ; 6 R_{0}\right)$ and $r \in\left\langle 0,24 R_{0} \vee 2^{-1} c_{2} R_{0}\right]$.

THEOREM A.2. ([CoW, Theorem III.1.2]). Let $E \subseteq B\left(x_{0} ; 2 R_{0}\right), J \subset X$, and $r: J$ $\rightarrow\left\langle 0,8 R_{0}\right]$. If $E \subseteq \bigcup_{x \in J} B(x ; r(x))$ then there exists a sequence $x_{1}, x_{2}, \ldots \in J$, possibly finitely many, such that the $B\left(x_{i} ; r\left(x_{i}\right)\right)$ are disjoint and $E \subseteq \bigcup_{i=1}^{\infty} B\left(x_{i} ; 5 r\left(x_{i}\right)\right)$

PROOF. This theorem is well known, see also [Ste, Section 1.7].

THEOREM A.3. ([CoW, Theorem III.1.3]). Let $\mathscr{O} \subseteq B\left(x_{0} ; 2 R_{0}\right)$ be open. Then there exist $x_{1}, x_{2}, \ldots \in \mathscr{O}$ and $\rho_{1}, \rho_{2}, \ldots \in\left\langle 0, R_{0}\right]$ such that $\mathscr{O}=\bigcup_{i=1}^{\infty} B\left(x_{i} ; \rho_{i}\right)$, each point of $\mathscr{O}$ is in at most $c_{d}^{5}$ balls $B\left(x_{i} ; \rho_{i}\right)$ and, moreover, $B\left(x_{i} ; 4 \rho_{i}\right) \cap(X \backslash \mathscr{O}) \neq \emptyset$ for all $i \in \mathbb{N}$. 
Proof. Apply Theorem A.2 with $J=\mathscr{O}$ and $r(x)=10^{-1} \rho(x, X \backslash \mathscr{O})$, the distance from $x$ to $X \backslash \mathscr{O}$. Then set $\rho_{i}=5 r\left(x_{i}\right)$, where the $x_{i}$ are obtained by Theorem A2. It remains to estimate a maximum on the overlap of the balls. Let $x \in \mathscr{O}$ and suppose $x \in B\left(x_{i} ; \rho_{i}\right)=B\left(x_{i} ; 2^{-1} \rho\left(x_{i}, X \backslash \mathscr{O}\right)\right)$. Arguing as in [CoW] one deduces that $2 \rho(x, X \backslash \mathscr{O}) / 3 \leq \rho\left(x_{i}, X \backslash \mathscr{O}\right) \leq 2 \rho(x, X \backslash \mathscr{O})$ and therefore $B\left(x_{i}, 10^{-1} \rho\left(x_{i}, X \backslash \mathscr{O}\right)\right)$ $\subset B(x, 6 \rho(x, X \backslash \mathscr{O}) / 5)$. Conversely, $B(x, 6 \rho(x, X \backslash \mathscr{O}) / 5) \subset B\left(x_{i}, 23 \rho\left(x_{i}, X \backslash \mathscr{O}\right) / 10\right)$. Then by the localized doubling property one has $\mu(B(x, 6 \rho(x, X \backslash \mathscr{O}) / 5)) \leq c_{d}^{5} \mu$ $\left(B\left(x_{i}, 10^{-1} \rho\left(x_{i}, X \backslash \mathscr{O}\right)\right)\right)$ and it follows from the disjointness of the balls $B\left(x_{i}, 10^{-1} \rho(x, X \backslash \mathscr{O})\right)$ that $x$ is in at most $c_{d}^{5}$ balls $B\left(x_{i} ; \rho_{i}\right)$.

The next lemma with its corollary are the key elements for the localization. We set $c_{4}=1+c_{d}^{4} \mu\left(B\left(x_{0} ; R_{0}\right)\right)^{-1}$.

LEMMA A.4. If $y \in B\left(x_{0} ; 5 R_{0}\right)$ and $r \in\left[2^{-1} R_{0}, 4 R_{0}\right]$ then $\mu(B(y ; r)) \geq c_{4}^{-1}$.

PRoOF. Since $B\left(x_{0} ; R_{0}\right) \subset B(y ; 12 r)$ one obtains by the localized doubling $\mu\left(B\left(x_{0} ; R_{0}\right)\right) \leq \mu(B(y ; 12 r)) \leq c_{d}^{4} \mu(B(y ; r))$.

For $\varphi \in L_{1}(X)$ with supp $\varphi \subset B\left(x_{0} ; R_{0}\right)$ define the local Hardy-Littlewood function $M \varphi: X \rightarrow[0, \infty]$ by

$$
(M \varphi)(x)=\sup _{\substack{r \in\left\{0,4 R_{0}\right] \\ y \in B(x ; r)}} \mu(B(y ; r))^{-1} \int_{B(y ; r)}|\varphi| .
$$

Moreover, define $\tilde{M} \varphi: X \rightarrow[0, \infty]$ by

$$
(\widetilde{M} \varphi)(x)=\sup _{r \in\left\{0.8 R_{0}\right]} \mu(B(x ; r))^{-1} \int_{B(x ; r)}|\varphi| .
$$

COROllary A.5. Let $\varphi \in L_{1}(X)$ with $\operatorname{supp} \varphi \subset B\left(x_{0} ; R_{0}\right)$. Then

$$
(M \varphi)(x) \leq c_{4}\|\varphi\|_{1}
$$

for all $x \in X \backslash B\left(x_{0} ; 2 R_{0}\right)$.

PROOF. We may assume that $\|\varphi\|_{1} \neq 0$ and $(M \varphi)(x) \neq 0$. Let $r \in\left\langle 0,4 R_{0}\right]$, $y \in B(x ; r)$ and suppose that $\int_{B(y ; r)}|\varphi| \neq 0$. Then $B(y ; r) \cap B\left(x_{0} ; R_{0}\right) \neq \emptyset$. Since $x \notin B\left(x_{0} ; 2 R_{0}\right)$ this implies that $r \geq 2^{-1} R_{0}$. Moreover, using again that $B(y ; r) \cap B\left(x_{0} ; R_{0}\right) \neq \emptyset$, it follows that $\rho\left(y ; x_{0}\right) \leq r+R_{0} \leq 5 R_{0}$ and $y \in B\left(x_{0} ; 5 R_{0}\right)$. Then $\mu(B(y ; r))^{-1} \int_{B(y ; r)}|\varphi| \leq c_{4} \int_{X}|\varphi|=c_{4}\|\varphi\|_{1}$ by Lemma A.4. So $(M \varphi)(x) \leq$ $c_{4}\|\varphi\|_{1}$. 
THEOREM A.6. ([CoW, Theorem III.2.1]). If $\varphi \in L_{1}(X)$ with $\operatorname{supp} \varphi \subset B\left(x_{0} ; R_{0}\right)$ and $\alpha \geq c_{4}\|\varphi\|_{1}$ then $\mu\left(E_{\alpha}\right) \leq c_{d}^{5} \alpha^{-1}\|\varphi\|_{1}$ and $E_{\alpha} \subset B\left(x_{0} ; 2 R_{0}\right)$, where $E_{\alpha}=\{x \in$ $X:(M \varphi)(x)>\alpha\}$.

PROOF. It follows from Corollary A.5 that $E_{\alpha} \subset B\left(x_{0} ; 2 R_{0}\right)$.

Next, if $x \in B\left(x_{0} ; 2 R_{0}\right), r \in\left\langle 0,4 R_{0}\right]$ and $y \in B(x ; r)$ then $B(y ; r) \subset B(x ; 2 r)$ and $\mu(B(x ; 2 r)) \leq \mu(B(y ; 3 r)) \leq c_{d}^{2} \mu(B(y ; r))$. So

$$
\mu(B(y ; r))^{-1} \int_{B(y ; r)}|\varphi| \leq c_{d}^{2} \mu(B(x ; 2 r))^{-1} \int_{B(x ; 2 r)}|\varphi| \leq c_{d}^{2}(\tilde{M} \varphi)(x)
$$

and $(M \varphi)(x) \leq c_{d}^{2}(\tilde{M} \varphi)(x)$ for all $x \in B\left(x_{0} ; 2 R_{0}\right)$. Therefore

$$
E_{\alpha} \subset\left\{x \in B\left(x_{0} ; 2 R_{0}\right):(\tilde{M} \varphi)(x)>c_{d}^{-2} \alpha\right\} .
$$

If $x \in E_{\alpha}$ then there exists an $r(x) \in\left\langle 0,8 R_{0}\right]$ such that $\mu(B(x ; r(x)))^{-1} \int_{B(x ; r(x))}|\varphi|>$ $c_{d}^{-2} \alpha$. Apply Theorem A.2 with $J=E=E_{\alpha}$. Then $\|\varphi\|_{1} \geq \sum_{i=1}^{\infty} \int_{B\left(x_{i} ; r\left(x_{i}\right)\right)}|\varphi| \geq$ $\sum_{i=1}^{\infty} c_{d}^{-2} \alpha \mu\left(B\left(x_{i} ; r\left(x_{i}\right)\right)\right) \geq \sum_{i=1}^{\infty} c_{d}^{-5} \alpha \mu\left(B\left(x_{i} ; 5 r\left(x_{i}\right)\right)\right) \geq c_{d}^{-5} \alpha \mu\left(E_{\alpha}\right)$.

THEOREM A.7. ([CoW, Theorem III.2.2 and Corollary III.2.3]). If $\varphi \in L_{1}(X)$ with $\operatorname{supp} \varphi \subset B\left(x_{0} ; R_{0}\right)$ and $\alpha \geq c_{4}\|\varphi\|_{1}$ then there exists a sequence $x_{1}, x_{2}, \ldots \in$ $B\left(x_{0} ; 2 R_{0}\right)$, possibly finite many, and $\rho_{1}, \rho_{2}, \ldots \in\left\langle 0, R_{0}\right]$ such that

I. $|\varphi(x)| \leq \alpha$ for almost every $x \in X \backslash \bigcup_{i=1}^{\infty} B\left(x_{i} ; \rho_{i}\right)$,

II. $\mu\left(B\left(x_{i} ; \rho_{i}\right)\right)^{-1} \int_{B\left(x_{i} ; \rho_{i}\right)}|\varphi| \leq c_{d}^{2} \alpha$ for all $i \in \mathbb{N}$,

III. $\sum_{i=1}^{\infty} \mu\left(B\left(x_{i} ; \rho_{i}\right)\right) \leq c_{d}^{10} \alpha^{-1}\|\varphi\|_{1}$,

IV. each point of $X$ is in at most $c_{d}^{5}$ balls $B\left(x_{i} ; \rho_{i}\right)$.

Moreover, there exist $\psi, \tau_{1}, \tau_{2}, \ldots \in L_{1}(X)$ such that

V. $\varphi=\psi+\sum_{i=1}^{\infty} \tau_{i}$,

VI. $|\psi(x)| \leq c_{d}^{7} \alpha$,

VII. $\|\psi\|_{1} \leq\left(1+c_{d}^{5}\right)\|\varphi\|_{1}$,

VIII. $\operatorname{supp} \tau_{i} \subseteq B\left(x_{i} ; \rho_{i}\right)$ for all $i \in \mathbb{N}$,

IX. $\int \tau_{i}=0$ for all $i \in \mathbb{N}$,

X. $\quad \sum_{i=1}^{\infty}\left\|\tau_{i}\right\|_{1} \leq 2 c_{d}^{5}\|\varphi\|_{1}$.

Proof. Set $\mathscr{O}=E_{\alpha}=\{x \in X:(M \varphi)(x)>\alpha\}$ and apply Theorem A.3. Then I follows from the Lebesgue differentiation theorem. For the proof of II, let $i \in \mathbb{N}$. Then $B\left(x_{i}, ; 4 \rho_{i}\right) \cap\left(X \backslash E_{\alpha}\right) \neq \emptyset$, so there exists a $y_{i} \in B\left(x_{i}, ; 4 \rho_{i}\right) \cap\left(X \backslash E_{\alpha}\right)$. Then $\rho\left(x_{i} ; y_{i}\right)<4 \rho_{i} \leq 4 R_{0}$ and $(M \varphi)\left(y_{i}\right) \leq \alpha$. Therefore

$$
\mu\left(B\left(x_{i} ; \rho_{i}\right)\right)^{-1} \int_{B\left(x_{i} ; \rho_{i}\right)}|\varphi| \leq c_{d}^{2} \mu\left(B\left(x_{i} ; 4 \rho_{i}\right)\right)^{-1} \int_{B\left(x_{i} ; 4 \rho_{i}\right)}|\varphi| \leq c_{d}^{2}(M \varphi)\left(y_{i}\right) \leq c_{d}^{2} \alpha .
$$

The other statements follow as in [CoW]. 
Proof OF Theorem A.1. Let $\varphi_{0} \in L_{1}(X) \cap L_{2}(X)$. Set $\varphi=\varphi_{0} \cdot 1_{B\left(x_{0} ; R_{0}\right)}$. Then $T \varphi_{0}=T \varphi$. If we can show that $\mu(\{x \in X:|(T \varphi)(x)|>\gamma\}) \leq A \gamma^{-1}\|\varphi\|_{1}$ for a suitable $A$, uniformly for all $\alpha>0$, then

$$
\begin{aligned}
\mu\left(\left\{x \in X:\left|\left(T \varphi_{0}\right)(x)\right|>\gamma\right\}\right) & =\mu(\{x \in X:|(T \varphi)(x)|>\gamma\}) \\
& \leq A \gamma^{-1}\|\varphi\|_{1} \leq A \gamma^{-1}\left\|\varphi_{0}\right\|_{1}
\end{aligned}
$$

and we have proved the theorem.

Let $\alpha>0$. If $\alpha<c_{4}\|\varphi\|_{1}$ then $\mu(\{x \in X:|(T \varphi)(x)|>\gamma\}) \leq \mu\left(B\left(x_{0} ; R_{0}\right)\right) \leq$ $c_{4} \mu\left(B\left(x_{0} ; R_{0}\right)\right) \alpha^{-1}\|\varphi\|_{1}$ since supp $T \varphi \subseteq B\left(x_{0} ; R_{0}\right)$.

So we may assume that $\alpha \geq c_{4}\|\alpha\|_{1}$. Now one can use Theorem $A 7$ and argue as in the proof of [CoW, Theorem III.2.4]. There is only one small step that needs care. It is in the estimates of the measure of the set $F_{\alpha}$ of all $x \in \bigcup_{i=1}^{\infty} B\left(x_{i} ; c_{2} \rho_{i}\right)$ for which $|(T \varphi)(x)| \geq 2^{-1} \alpha$. Let $n \in \mathbb{N}_{0}$ be such that $2^{n} \leq c_{2} \leq 2^{n+1}$. Then the localized doubling property gives $\mu\left(B\left(x_{i} ; c_{2} \rho_{i}\right)\right) \leq c_{d}^{n+1} \mu\left(B\left(x_{i} ; \rho_{i}\right)\right)$. So by Theorem A7.III one deduces that $\mu\left(F_{\alpha}\right) \leq \mu\left(\bigcup_{i=1}^{\infty} B\left(x_{i} ; c_{2} \rho_{i}\right)\right) \leq c_{d}^{n+1} \mu\left(\bigcup_{i=1}^{\infty} B\left(x_{i} ; \rho_{i}\right)\right) \leq c_{d}^{n+11} \alpha^{-1}\|\varphi\|_{1}$. Since $n$ depends only on $c_{2}$ one obtains the required uniform bounds.

\section{References}

[ADM] D. Albrecht, X. Duong and A. McIntosh, 'Operator theory and harmonic analysis', in: Instructional workshop on analysis and geometry, part III, vol. 34 of Proceedings of the Centre for Mathematics and its Applications, Australian National University, Canberra 1996, 77-136.

[Aus] P. Auscher, 'Regularity theorems and heat kernel for elliptic operators', J. London Math. Soc. 54 (1996), 284-296.

[ACT] P. Auscher, T. Coulhon and P. Tchamitchian, 'Absence de principe du maximum pour certaines équations paraboliques complexes', Colloq. Math. 71 (1996), 87-95.

[AMT] P. Auscher, A. McIntosh and P. Tchamitchian, 'Heat kernels of second order complex elliptic operators and their applications', Research Report 94-164, (Maquarie University, Sydney, 1994).

[Bur] R. J. Burns, Sobolev spaces on Lie groups ( $\mathrm{PhD}$ thesis, The Australian National University, Canberra, 1991).

[BER] R. J. Burns, A. F. M. ter Elst and D. W. Robinson, ' $L_{p}$-regularity of subelliptic operators on Lie groups', J. Operator Theory 31 (1994), 165-187.

[BuB] P. L. Butzer and H. Berens, Semi-groups of operators and approximation, Grundlehren Math. Wiss. 145 (Springer, Berlin, 1967).

[CoW] R. R. Coifman and G. Weiss, Analyse harmonique non-commutative sur certains espaces homogénes, Lecture Notes in Math. 242 (Springer, Berlin, 1971).

[CDMY] M. Cowling, I. Doust, A. McIntosh and A. Yagi, 'Banach space operators with a bounded $H^{\infty}$ functional calculus', J. Austral. Math. Soc. (Series A) 60 (1996), 51-89.

[DuR] X. T. Duong and D. W. Robinson, 'Semigroup kernels, Poisson bounds and holomorphic functional calculus', J. Funct. Anal. 142 (1996), 89-129. 
[EIR1] A. F. M. ter Elst and D. W. Robinson, 'Subelliptic operators on Lie groups: regularity', J. Austral. Math. Soc. (Series A) 57 (1994), 179-229.

[EIR2] _ - 'Subcoercivity and subelliptic operators on Lie groups I: Free nilpotent groups', Potential Anal. 3 (1994), 283-337.

[ElR3] _ _ 'Functional analysis of subelliptic operators on Lie groups', J. Operator Theory 31 (1994), 277-301.

[EIR4] — 'Reduced heat kernels on nilpotent Lie groups', Comm. Math. Phys. 173 (1995), 475-511.

[EIR5] — - 'On Kato's square root problem', Hokkaido Math. J. 26 (1997), 365-376.

[EIR6] — - 'Second-order subelliptic operators on Lie groups I: complex uniformly continuous principal coefficients', Research Report MRR 035-96, (The Australian National University, Canberra, 1996).

[EIR7] _ _ 'High order divergence-form elliptic operators on Lie groups', Bull. Austral. Math. Soc. 55 (1997), 335-348.

[ElR8] — _ 'Second-order subelliptic operators on Lie groups II: real measurable principal coefficients', Research Report MRR 037-96 (The Australian National University, Canberra, Australia, 1996).

[Gia1] M. Giaquinta, Multiple integrals in the calculus of variations and nonlinear elliptic systems, Ann. of Math. Stud. 105 (Princeton University Press, Princeton, 1983).

[Gia2] _-, Introduction to regularity theory for nonlinear elliptic systems, Lectures in Math. ETH Zürich (Birkhäuser, Basel, 1993).

[Kat1] T. Kato, 'Fractional powers of dissipative operators', J. Math. Soc. Japan 13 (1961), 246274.

[Kat2] —, Perturbation theory for linear operators, 2nd edition, Grundlehren Math. Wiss. 132 (Springer, Berlin, 1984).

[Kor] Y. A. Kordyukov, ' $L$-Theory of elliptic differential operators on manifolds of bounded geometry', Acta Appl. Math. 23 (1991), 223-260.

[Lio] J. L. Lions, 'Espaces d'interpolation et domaines de puissances fractionnaires d'opérateurs', J. Math. Soc. Japan 14 (1962), 233-241.

[LiM] J. L. Lions and E. Magenes, Non-homogeneous boundary value problems and applications, Volume I, Grundlehren Math. Wiss. 181 (Springer, Berlin, 1972).

[McI] A. McIntosh, 'Square roots of elliptic operators', J. Funct. Anal. 61 (1985), 307-327.

[Rob] D. W. Robinson, Elliptic operators and Lie groups, Oxford Math. Monographs (Oxford University Press, Oxford, 1991).

[Ste] E. M. Stein, Singular integrals and differential properties of functions, Princeton Math. Series 30 (Princeton University Press, Princeton, 1970).

[Tay] M. E. Taylor, Pseudodifferential operators, Princeton Math. Series 34 (Princeton University Press, Princeton, 1981).

[Tri] H. Triebel, Interpolation theory, function spaces, differential operators (North-Holland, Amsterdam, 1978).

[VSC] N. T. Varopoulos, L. Saloff-Coste and T. Coulhon, Analysis and geometry on groups, Cambridge Tracts in Math. 100 (Cambridge University Press, Cambridge, 1992). 
Department of Mathematics and Computing Science

Eindhoven University of Technology

P.O. Box 513

$5600 \mathrm{MB}$ Eindhoven

The Netherlands

e-mail: terelst@win.tue.nl
Centre for Mathematics and its Applications School of Mathematical Sciences Australian National University Canberra, ACT 0200 Australia e-mail: derek.robinson@anu.edu.au 